\title{
HILSUM-SKANDALIS MAPS AS FROBENIUS ADJUNCTIONS WITH APPLICATION TO GEOMETRIC MORPHISMS
}

\author{
CHRISTOPHER TOWNSEND
}

\begin{abstract}
Hilsum-Skandalis maps, from differential geometry, are studied in the context of a cartesian category. It is shown that Hilsum-Skandalis maps can be represented as stably Frobenius adjunctions. This leads to a new and more general proof that Hilsum-Skandalis maps represent a universal way of inverting essential equivalences between internal groupoids.

To prove the representation theorem, a new characterisation of the connected components adjunction of any internal groupoid is given. The characterisation is that the adjunction is covered by a stable Frobenius adjunction that is a slice and whose right adjoint is monadic.

Geometric morphisms can be represented as stably Frobenius adjunctions. As applications of the study we show how it is easy to recover properties of geometric morphisms, seeing them as aspects of properties of stably Frobenius adjunctions.
\end{abstract}

\section{INTRODUCTION}

We provide a categorical account of Hilsum-Skandalis maps and establish that key results about them can be proved in the general context of a cartesian category $\mathcal{C}$, provided the domain and codomain groupoids are restricted to those that have connected components adjunctions that are stably Frobenius. The restriction is not a strong one as most groupoids used in application satisfy this property. One key result is that Hilsum-Skandalis maps form the morphisms of a category of fractions, inverting essential euivalences between internal groupoids. This is shown by providing a new result which is that Hilsum-Skandalis maps can be represented as stably Frobenius adjunctions over $\mathcal{C}$.

Along the way, in order to ease the discussion of categories of objects with groupoid actions, we provide a new categorical description of the connected components adjunction for internal groupoids which should be of general interest. The result is that an adjunction $\Sigma_{\mathcal{D}} \dashv \mathcal{D}^{*}: \mathcal{D} \rightleftarrows \mathcal{C}$ is equivalent to a connected components adjunction if and only if it is covered by a slice of $\mathcal{C}$, with a covering adjunction that is stably Frobenius and whose right adjoint is monadic.

A number of applications are given which show how the techniques developed can be applied to recover known aspects of the theory of geometric morphisms. It is shown how the pullback of bounded geometric morphisms can be described by using the product of localic groupoids and a proof is given of pullback stable hyperconnected-localic factorization.

All our categories are cartesian (i.e. they have finite limits). 


\section{Summary OF CONTENTS}

Our first few sections are categorical preliminaries covering properties of categories of adjunctions over a base category, Frobenius reciprocity, monadicity and effective descent. Then there is a section on groupoids which provides new characterisations of the connected components adjunction, in terms of these categorical concepts (adjunctions over a base, monadicity, Frobenius reciprocity and effective descent).

We then define Hilsum-Skandalis maps and show that stably Frobenius adjunctions give rise to them. In fact by restricting to what we have called stably Frobenius groupoids, which are then introduced, we see that all Hilsum-Skandalis maps arise in this way. This is the main result of the paper: Hilsum-Skandalis maps are essentially the same thing as stably Frobenius adjunctions. Along the way there is some discussion about internal essential equivalences between internal groupoids, showing that they can be used to characterise Hilsum-Skandalis maps in the usual manner. We then focus on applications of the main result and show relative to an arbitrary cartesian category that:

(i) Hilsum-Skandalis maps universally invert essential equivalences,

(ii) stably Frobenius groupoids internal to a category of $\mathbb{G}$-objects are (up to a natural equivalence) the same thing as Hilsum-Skandalis maps to $\mathbb{G}$ (this is achieved with a new categorically flavoured construction of groupoid semidirect product) and

(iii) connected component adjunctions of stably Frobenius groupoids are pullback stable.

Finally the paper focuses on applications to geometric morphisms, recalling that geometric morphisms can be represented as certain stably Frobenius adjunctions. The categorical characterisation of connected component adjunctions is applied to characterise bounded geometric morphisms, from which basic results about localic and bounded geometric morphisms follow. The pullback of connected component adjunctions is used to describe the pullback of bounded geometric morphisms. An account of the hyperconnected-localic factorisation is given.

\section{Preliminaries}

3.1. Notation for pullback. We will assume that all our categories are cartesian and start with some notation. If $f: Y \longrightarrow X$ is a morphism of a category $\mathcal{C}$ then we use $Y_{f}$ as notation for $f$ when considered as an object of the slice category $\mathcal{C} / X$. Observe that $(\mathcal{C} / X) / Y_{f}$ is isomorphic to $\mathcal{C} / Y$. We use $Y_{X}$ as notation for the object $\pi_{1}: X \times Y \longrightarrow X$. For any morphism $f: Y \longrightarrow X$ of a cartesian category $\mathcal{C}$ there is a pullback adjunction $\Sigma_{f} \dashv f^{*}: \mathcal{C} / Y \longrightarrow \mathcal{C} / X$; if $Z_{g}$ is an object of $\mathcal{C} / Y$ then $\Sigma_{f}\left(Z_{g}\right)$ is defined to be $Z_{f g}$. In the case $X=1$ we use $\Sigma_{Y} \dashv Y^{*}$ for the pullback adjunction; $\Sigma_{Y}$ reflects isomorphisms and creates coequalizers.

3.2. Adjunctions over a base category. We shall frequently be discussing results relative to a base category $\mathcal{C}$. If we have two other categories $\mathcal{D}_{i}, i=1,2$, each equiped with an adjunction $\Sigma_{\mathcal{D}_{i}} \dashv \mathcal{D}_{i}^{*}: \mathcal{D}_{i} \rightleftarrows \mathcal{C}$ back to $\mathcal{C}$ (so $\Sigma_{\mathcal{D}_{i}}$ goes from $\mathcal{D}_{i}$ to $\mathcal{C})$ then an adjunction $L \dashv R: \mathcal{D}_{1} \rightleftarrows \mathcal{D}_{2}$ is said to be over $\mathcal{C}$ provided there is a natural isomorphism $\tau: \Sigma_{\mathcal{D}_{2}} L \stackrel{\cong}{\longrightarrow} \Sigma_{\mathcal{D}_{1}}$. The morphisms of the category of adjunctions over $\mathcal{C}$ consist of natural transformations on left adjoints that commute with the natural isomorphisms $\tau$ in the obvious manner; i.e. if $\alpha: L_{1} \longrightarrow L_{2}$ then $\tau_{1}: \Sigma_{\mathcal{D}_{2}} L_{1} \longrightarrow \Sigma_{\mathcal{D}_{1}}$ factors as $\Sigma_{\mathcal{D}_{2}} L_{1} \stackrel{\Sigma_{\mathcal{D}_{2}} \alpha}{\longrightarrow} \Sigma_{\mathcal{D}_{2}} L_{2} \stackrel{\tau_{2}}{\longrightarrow} \Sigma_{\mathcal{D}_{1}}$. 
If $X$ is an object of $\mathcal{C}$ and we assert that an adjunction $L \dashv R: \mathcal{C} / X \rightleftarrows \mathcal{D}$ is over $\mathcal{C}$ then we mean that there is a natural isomorphism $\alpha: \Sigma_{\mathcal{D}} L \stackrel{\cong}{\longrightarrow} \Sigma_{X}$. Notice that for the case $X=1$ having such an adjunction means that there is a splitting of the given adjunction $\Sigma_{\mathcal{D}} \dashv \mathcal{D}^{*}$ back to $\mathcal{C}$.

Given an adjunction $L \dashv R: \mathcal{D} \rightleftarrows \mathcal{C}$, then for any object $X$ of $\mathcal{C}$ there is a sliced adjunction $L_{X} \dashv R_{X}: \mathcal{D} / R X \rightleftarrows \mathcal{C} / X ; L_{X}(g)=$ "the adjoint transpose of $g^{\prime}$ and $R_{X}\left(Y_{f}\right)=R Y_{R f}$.

It is a trivial from the definition of adjoint transpose to see that any adjunction $L \dashv R$ (between cartesian categories) factors through its slice at $L 1$ :

$$
\mathcal{D} \underset{\eta_{1}^{*}}{\stackrel{\Sigma_{\eta_{1}}}{\rightleftarrows}} \mathcal{D} / R L 1 \underset{R_{L 1}}{\stackrel{L_{L 1}}{\rightleftarrows}} \mathcal{C} / L 1 \underset{L 1^{*}}{\stackrel{\Sigma_{L 1}}{\rightleftarrows}} \mathcal{C}
$$

and similar observations can be exploited to relate cetain adjunctions over $\mathcal{C}$ to adjunctions that are splittings:

Lemma 3.1. Given an adjunction $\Sigma_{\mathcal{D}} \dashv \mathcal{D}^{*}: \mathcal{D} \rightleftarrows \mathcal{C}$ and an object $X$ of $\mathcal{C}$ then the category of adjunctions $L \dashv R: \mathcal{C} / X \rightleftarrows \mathcal{D}$ over $\mathcal{C}$ is equivalent to the category of adjunctions $L^{\prime} \dashv R^{\prime}: \mathcal{C} / X \rightleftarrows \mathcal{D} / \mathcal{D}^{*} X$ over $\mathcal{C} / X$. Further, under this equivalence, $L^{\prime} 1=L 1 \stackrel{\psi}{\longrightarrow} \mathcal{D}^{*} X$ where $\psi$ is the adjont transpose, across $\Sigma_{\mathcal{D}} \dashv \mathcal{D}^{*}$, of the isomorphism $\alpha_{1}: \Sigma_{\mathcal{D}} L 1 \stackrel{\cong}{\longrightarrow} \Sigma_{X} 1=X$ that exists by assumption that $L \dashv R$ is over $\mathcal{C}$.

Proof. Send $L \dashv R$ to $L_{\mathcal{D}^{*} X} \Sigma_{\Delta_{X}} \dashv \Delta_{X}^{*} R_{\mathcal{D}^{*} X}$. The adjunction $L_{\mathcal{D}^{*} X} \Sigma_{\Delta_{X}} \dashv$ $\Delta_{X}^{*} R_{\mathcal{D}^{*} X}$ is over $\mathcal{C} / X$ because $\left(\Sigma_{X}\right)_{X} \cong\left(\Sigma_{\mathcal{D}}\right)_{X} L_{\mathcal{D}^{*} X}$ (if $\Sigma_{X} \cong \Sigma_{\mathcal{D}} L$ ) and $I d_{\mathcal{C} / X}$ factors as $\Sigma_{\pi_{2}} \Sigma_{\Delta_{X}}$. In the other direction send $L^{\prime} \dashv R^{\prime}$ to $\Sigma_{\mathcal{D}^{*} X} L^{\prime} \dashv R^{\prime}\left(\mathcal{D}^{*} X\right)^{*}$. Observe that

$$
\mathcal{C} / X \times X \cong(\mathcal{C} / X) / X^{*} X \cong(\mathcal{C} / X) / R \mathcal{D}^{*} X\left(^{*}\right)
$$

Note that the adjoint transpose of the morphism $\Delta_{X}: 1 \longrightarrow X^{*} X$ of $\mathcal{C} / X$ under the adjunction $\Sigma_{X} \dashv X^{*}$ is the identity on $X$ and so the 'further' part follows by uniquenss of adjoint transpose, taking the isomorphisms of categories $(*)$ into consideration.

Being able to relate general adjunctions over $\mathcal{C}$ with sliced domain, to splittings of adjunctions back to $\mathcal{C}$ will ease proofs considerably as the split case is generally easy. We shall see a number of examples of this in what follows.

\section{Frobenius Reciprocity}

An adjunction $L \dashv R: \mathcal{D} \rightleftarrows \mathcal{C}$ satisfies Frobenius reciprocity (or is Frobenius) provided the map

$$
L(W \times R X) \stackrel{\left(L \pi_{1}, L \pi_{2}\right)}{\longrightarrow} L W \times L R X \stackrel{I d_{L W} \times \epsilon_{X}}{\longrightarrow} L W \times X
$$

is an isomorphism for all objects $W$ and $X$ of $\mathcal{D}$ and $\mathcal{C}$ respectively, where $\epsilon$ is the counit of the adjunction. Between cartesian closed categories an adjunction satisfies Frobenius reciprocity if and only if the right adjoint preserves exponentials $\left(R\left(X^{Y}\right) \cong R X^{R Y}\right)$, however the condition is also relevant in the general context of cartesian categories, a striking example being the category of locales. Categories of locales (relative to a topos) are not cartesian closed but the Frobenius condition on adjunctions between them can be used to characterise geometric morphsms, T10. Being Frobenius is two conditions away from being an equivalence: 
Lemma 4.1. A Frobenius adjunction $L \dashv R: \mathcal{D} \rightleftarrows \mathcal{C}$ is an equivalence iff $L 1 \cong 1$ and $\eta_{W}$ is a regular monomorphism for each object $W$ of $\mathcal{D}$ then the adjunction is an equivalence.

Proof. If the adjunction is an equivalence the unit is an isomorphism and $L 1 \cong 1$, so one way round is clear. In the other direction, $L R X \cong L(1 \times R X) \cong L 1 \times X \cong X$; from which the counit $\epsilon$ is seen to be an isomorphism. For each object $W$ there is an equalizer diagram $W \stackrel{\eta_{W}}{\longrightarrow} R L W \underset{b}{\stackrel{a}{\longrightarrow}} W^{\prime}$ for some $a, b$ and $W^{\prime}$. Then $L a=L b$ since $L \eta_{W}$ is an isomorphism using the triangular identities and the fact that $\varepsilon$ is an isomorphism. But if $L a=L b$ then they have equal adjoint transposes; that is, $\eta_{W^{\prime}} a=\eta_{W^{\prime}} b$ and so $a=b$ since $\eta_{W^{\prime}}$ is a monomorphism. Hence $\eta_{W}$ is an isomorphism and the adjunction in fact is an equivalence.

If $X$ is an object of $\mathcal{C}$, then $L \dashv R$ is Frobenius at $X$ provided $L_{X} \dashv R_{X}$ satisfies Frobenius reciprocity and is stably Frobenius if $L_{X} \dashv R_{X}$ satisfies Frobenius reciprocity for every object $X$ of $\mathcal{C}$.

It is easy to see that an adjunction $L \dashv R: \mathcal{D} \rightleftarrows \mathcal{C}$ is stably Frobenius if and only if for any pair of morphisms $g: W \longrightarrow R X$ and $f: Y \longrightarrow X$, the canonical map $L\left(W \times_{R X} R Y\right) \longrightarrow L W \times_{X} Y$ is an isomorphism, where the codomain is the pullback of $f$ along the adjoint transpose of $g$. For example any morphism $f: X \longrightarrow Y$ of a cartesian category $\mathcal{C}$ the pullback adjunction $\Sigma_{f} \dashv$ $f^{*}: \mathcal{C} / X \longrightarrow \mathcal{C} / Y$ is stably Frobenius. The composition of two (stably) Frobenius adjunctions is (stably) Frobenius.

The next lemma provides an example of the usefulness of being able to relate adjunctions over $\mathcal{C}$ to split adjunctions. We will see towards the end of this paper that Part 2 can be viewed as the categorical essence of the well known fact that if the composite $f g$ of two geometric morphisms is localic, then $g$ is localic.

Lemma 4.2. Let $\Sigma_{\mathcal{D}} \dashv \mathcal{D}^{*}: \mathcal{D} \rightleftarrows \mathcal{C}$ be an adjunction.

1. If $L \dashv R: \mathcal{C} \rightleftarrows \mathcal{D}$ is over $\mathcal{C}$ and Frobenius at $L 1$, then $L_{1}: \mathcal{C} / 1 \longrightarrow \mathcal{D} / L 1$, is an equivalence.

2. If $X$ is an object of $\mathcal{C}$ and $L \dashv R: \mathcal{C} / X \rightleftarrows \mathcal{D}$ is an adjunction over $\mathcal{C}$ that is Frobenius at $\mathcal{D}^{*} X$ then $L_{1}: \mathcal{C} / X \longrightarrow \mathcal{D} / L 1$ is an equivalence.

Proof. 1. We need to show that the adjunction

$$
\mathcal{C} \underset{\eta_{1}^{*}}{\stackrel{\Sigma_{\eta_{1}}}{\rightleftarrows}} \mathcal{C} / R L 1 \underset{R_{L 1}}{\stackrel{L_{L 1}}{\rightleftarrows}} \mathcal{D} / L 1
$$

is an equivalence. Lemma 4.1 can be applied as the adjunction satisfies Frobenius reciprocity by assumption (and the fact that the composition of two Frobenius adjunctions is Frobenius). Since $L_{1} 1 \cong 1$ we are just required to check that the unit of this adjunction is a regular monomorphism. At $X$, an object of $\mathcal{C}$, the unit is $X \stackrel{\left(!^{X}, \eta_{X}\right)}{\longrightarrow} 1 \times_{R L 1} R L X$ where the pullback is of $R L\left(!^{X}: X \longrightarrow 1\right)$ along $\eta_{1}$ : $1 \longrightarrow R L 1$. Now because $L \dashv R$ is over $\mathcal{C}$ the composite adjunction $\Sigma_{\mathcal{D}} L \dashv R \mathcal{D}^{*}$ is an equivalence and so its unit, $X \stackrel{\eta_{X}}{\longrightarrow} R L X \stackrel{R \eta_{L X}^{D}}{\longrightarrow} R \mathcal{D}^{*} \Sigma_{\mathcal{D}} L X$ is an isomorphism which implies that $\eta_{X}$ is a split monomorphism (it is split by $\left.\left(R \eta_{L X}^{\mathcal{D}} \eta_{X}\right)^{-1} R \eta_{L X}^{\mathcal{D}}\right)$. This is sufficient to show that $\left(!^{X}, \eta_{X}\right)$ is a regular monomorphism.

2. By 1. and Lemma 3.1 we know that

$$
\left(L_{\mathcal{D}^{*} X} \Sigma_{\Delta_{X}}\right)_{1}:(\mathcal{C} / X) / 1 \longrightarrow\left(\mathcal{D} / \mathcal{D}^{*} X\right) /\left(L 1 \stackrel{\psi}{\longrightarrow} \mathcal{D}^{*} X\right)
$$


is an equivalence. But $\left(\mathcal{D} / \mathcal{D}^{*} X\right) /\left(L 1 \stackrel{\psi}{\longrightarrow} \mathcal{D}^{*} X\right) \cong \mathcal{D} / L 1$.

For our final introductory lemma we expose a close relationship between morphisms and natural transformations that arises once we restrict to stably Frobenius adjunctions.

Lemma 4.3. Let $\Sigma_{\mathcal{D}} \dashv \mathcal{D}^{*}: \mathcal{D} \rightleftarrows \mathcal{C}$ an adjunction, $X$ an object of $\mathcal{C}$ and $L_{i} \dashv R_{i}: \mathcal{C} / X \rightleftarrows \mathcal{D}$ with $i=1,2$, two adjunctions over $\mathcal{C}$, both Frobenius at $\mathcal{D}^{*} X$. Let $\psi_{i}: L_{i} 1 \longrightarrow \mathcal{D}^{*} X$ be the adjoint transpose of the isomorphism $\tau_{1}^{i}$ : $\Sigma_{\mathcal{D}} L_{i} 1 \stackrel{\cong}{\longrightarrow} \Sigma_{X} 1$ that exists by assumption that $L_{i} \dashv R_{i}$ is over $\mathcal{C}$. Then there is a bijection between morphisms $\mathcal{D} / \mathcal{D}^{*} X\left(\left(L_{1} 1\right)_{\psi_{1}},\left(L_{2} 1\right)_{\psi_{2}}\right)$ and morphisms $\left(L_{1} \dashv\right.$ $\left.R_{1}\right) \longrightarrow\left(L_{2} \dashv R_{2}\right)$ over $\mathcal{C}$.

Proof. Any $\alpha: L_{1} \longrightarrow L_{2}$ gives rise to a map $\alpha_{1}: L_{1} 1 \longrightarrow L_{2} 1$ which is over $\mathcal{D}^{*} X$ if $\alpha$ is over $\mathcal{C}$. In the other direction any object $Y_{f}$ of $\mathcal{C} / X$ is isomorphic to the pullback $1 \times_{X^{*} X} X^{*} Y$ (i.e. the pullback of $I d_{X} \times f$ along $\Delta_{X}$ ). As $L_{i} \dashv R_{i}$ is Frobenius at $\mathcal{D}^{*} X$ and over $\mathcal{C}$ there are isomorphisms:

$$
L_{i}\left(Y_{f}\right) \cong L_{i}\left(1 \times_{X^{*} X} X^{*} Y\right) \cong L_{i}\left(1 \times_{R_{i} \mathcal{D}^{*} X} R_{i} \mathcal{D}^{*} Y\right) \cong L_{i} 1 \times_{\mathcal{D}^{*} X} \mathcal{D}^{*} Y(\star)
$$

where the last pullback is $I d \times \mathcal{D}^{*} f$ along $\psi_{i}$ (recall that $\Delta_{X}$ is the unit of $\Sigma_{X} \dashv X^{*}$ evaluated at 1). Using these isomorphisms, any map $a: L_{1} 1 \longrightarrow L_{2} 1$ over $\mathcal{D}^{*} X$ gives rise to a natural transformation $\hat{a}: L_{1} \longrightarrow L_{2}$. To see that this natural transformation is over $\mathcal{C}$ observe that $\tau_{Y_{f}}^{i}$ factors as

$$
\Sigma_{\mathcal{D}} L_{i}\left(Y_{f}\right) \stackrel{\Sigma_{\mathcal{D}}(\star)}{\longrightarrow} \Sigma_{\mathcal{D}}\left(L_{i} 1 \times_{\mathcal{D}^{*} X} \mathcal{D}^{*} Y\right) \stackrel{\left(\Sigma_{\mathcal{D}} \pi_{1}, \epsilon_{Y}^{\mathcal{D}} \Sigma_{\mathcal{D}} \pi_{2}\right)}{\longrightarrow} \Sigma_{\mathcal{D}} L_{i} 1 \times_{X} Y \stackrel{\pi_{2}}{\longrightarrow} Y
$$

where $\epsilon^{\mathcal{D}}$ is the counit of $\Sigma_{\mathcal{D}} \dashv \mathcal{D}^{*}$.

That $\hat{a}_{1}=a$ is clear from construct and that $\alpha_{Y_{f}}=\left(\hat{\alpha_{1}}\right)_{Y_{f}}$ for each $Y_{f}$ follows by naturality of $\alpha$ at ! : $Y_{f} \longrightarrow 1$ and the fact that $\alpha$ is over $\mathcal{C}$.

\section{MonADiCITY}

This section consists of a short paragraph where we recall some basic facts about monadic functors.

Beck's monadicity theorem proves that a functor $R$ is monadic iff it (i) has a left adjoint, (ii) reflects isomorphisms and (iii) has and preserves coequalizers for any $R$ split pair of arrows (that is, for any parallel pair of arrows $f, g: X \Longrightarrow Y$ such that $R f, R g$ is part of a split coequalizer diagram with coequalizer, $\bar{q}: R Y \longrightarrow \bar{Q}$, there exists a coequalizer $q: X \longrightarrow Q$ of $f, g$ such that the canonical map $\bar{Q} \longrightarrow R Q$ is an isomorphism). From this characterisation of monadicity it is clear that a functor $R: \mathcal{C} \longrightarrow \mathcal{D}$ is monadic iff $R_{X}$ is monadic for every $X$; i.e. monadicity is slice stable. If $R: \mathcal{C} \longrightarrow \mathcal{D}$ is split by $S: \mathcal{D} \longrightarrow \mathcal{C}$ (i.e. we are given a natural isomorphism $S R \cong I d_{\mathcal{C}}$ ), then $R$ is monadic if it has a left adjoint (essentially because split coequalizers are preserved by any functor; applied here to both $S$ and $R$ ). If we have two functors $R: \mathcal{C} \longrightarrow \mathcal{D}$ and $R^{\prime}: \mathcal{D} \longrightarrow \mathcal{D}^{\prime}$, both with left adjoints, then provided $R^{\prime}$ reflects isomorphisms, $R$ is monadic whenever $R^{\prime} R$ is monadic. In particular, monadicity descends along monadic functors; i.e. if both $R^{\prime} R$ and $R^{\prime}$ are monadic then so is $R$. On the other hand if $R^{\prime}$ has a left adjoint and is split and $R$ is monadic then $R^{\prime} R$ is monadic; so for split $R^{\prime}$ (with a left adjoint) we have that $R^{\prime} R$ is monadic iff $R$ is monadic. 


\section{EFFECTIVE DESCENT}

If $f: X \longrightarrow Y$ is a morphism of $\mathcal{C}$, then $f$ is an effective descent morphism if the functor $f^{*}: \mathcal{C} / Y \longrightarrow \mathcal{C} / X$ is monadic. An effective descent morphism is necessarily a regular epimorphism (the kernel pair of $f$ is $f^{*}$-split) and the property of being an effective descent morphism is pullback stable by our observation that monadicity is slice stable. Further, from our observations about monadicity, we known that split epimorphisms are effective descent morphisms and that for any pair of composable morphisms $f, g$, if $f g$ and $g$ are effective descent morphisms then so is $f$. We also know that if $g$ is a split epimorphism then $f g$ is an effective descent morphism if $f$ is.

The next lemma shows that effective descent morphisms interact well with Frobenius adjunctions over a base category $\mathcal{C}$ and is another example of an application of Lemma 3.1;

Lemma 6.1. Let $\Sigma_{\mathcal{D}} \dashv \mathcal{D}^{*}: \mathcal{D} \rightleftarrows \mathcal{C}$ be an adjunction.

1. Given an adjunction $L \dashv R: \mathcal{C} \rightleftarrows \mathcal{D}$ over $\mathcal{C}$ that satisfies Frobenius reciprocity, if $!^{W}: W \longrightarrow 1$ is an effect descent morphism in $\mathcal{D}$ then $!^{R W}: R W \longrightarrow 1$ is an effective descent morphism of $\mathcal{C}$.

2. Let $X$ be an object of $\mathcal{C}$. If $L \dashv R: \mathcal{C} / X \rightleftarrows \mathcal{D}$ is over $\mathcal{C}$ which is Frobenius at $\mathcal{D}^{*} X$ and $!^{W}: W \longrightarrow 1$ is an effect descent morphism in $\mathcal{D}$, then $R W$ is an effective descent morphism of $\mathcal{C}$.

Proof. 1. (a) If $\phi: U \longrightarrow V$ is a morphism of $\mathcal{C}$ such that $U \times R W \stackrel{\phi \times I d_{R W}}{\longrightarrow} V \times R W$ is an isomorophism then, by applying $L$ and the Frobenius reciprocity assumption we have that $L U \times W \stackrel{L \phi \times I d_{W}}{\longrightarrow} L V \times W$ is an isomorphism. Since $!^{W}$ is an effective descent morphism, $L \phi$ is therefore an isomorphism. But $\Sigma_{\mathcal{D}} L \cong I d_{\mathcal{C}}$ by assumption that $L \dashv R$ is over $\mathcal{C}$ and so $\phi$ is an isomorphism. (b) Say $U \underset{g}{\stackrel{f}{\longrightarrow}} V$ is a pair of morphisms in $\mathcal{C}$ such that there is $U_{R W} \underset{\frac{g \times I d}{l}}{\stackrel{f \times I d}{\rightleftarrows}} V_{R W} \underset{n}{\stackrel{m}{\rightleftarrows}} K_{k}$, a split coequalizer diagram in $\mathcal{C} / R W$. By taking adjoint transpose across $L \dashv R$ and applying Frobenius reciprocity we obtain $L U_{W} \underset{\stackrel{L g \times I d}{\rightleftarrows}}{\stackrel{L f \times I d}{\rightleftarrows}} L V_{W} \stackrel{m}{\rightleftarrows}(L K)_{\hat{k}}$, a split coequalizer diagram in $\mathcal{D} / W$. Since $!^{W}$ is an effective descent morphism, there exists $L V \stackrel{q}{\longrightarrow} Q$, a coequalizer of $L f, L g$ such that the canonical map $L K \longrightarrow Q \times W$ is an isomorphism. By applying $\Sigma_{\mathcal{D}}$, a left adjoint, to the coequalizer diagram that defines $Q$, and recalling that $\Sigma_{\mathcal{D}} L \cong I d_{\mathcal{C}}$ by assumption, we have that $\Sigma_{\mathcal{D}} Q$ is a coequalizer of $f, g$. But, further, $L \Sigma_{\mathcal{D}} Q \cong Q$ as $L$ preserves coequalizers and $R \mathcal{D}^{*} \cong I d_{\mathcal{C}}$ and so the following series of natural isomorphisms in $N$, an object of $\mathcal{C}$, shows that 


$$
\begin{aligned}
\Sigma_{\mathcal{D}} Q \times R W \cong K & \\
\mathcal{C}(K, N) & \cong \mathcal{C}\left(K, R \mathcal{D}^{*} N\right) \\
& \cong \mathcal{D}\left(L K, \mathcal{D}^{*} N\right) \\
& \cong \mathcal{D}\left(Q \times W, \mathcal{D}^{*} N\right) \\
& \cong \mathcal{D}\left(L \Sigma_{\mathcal{D}} Q \times W, \mathcal{D}^{*} N\right) \\
& \cong \mathcal{D}\left(L\left(\Sigma_{\mathcal{D}} Q \times R W\right), \mathcal{D}^{*} N\right) \\
& \cong \mathcal{C}\left(\Sigma_{\mathcal{D}} Q \times R W, R \mathcal{D}^{*} N\right) \cong \mathcal{C}\left(\Sigma_{\mathcal{D}} Q \times R W, N\right)
\end{aligned}
$$

Establishing (a) and (b) proves 1., by application of Beck's monadicity theorem.

2. Firstly by our observation that monadicity is slice stable we know that !: $W_{\mathcal{D}^{*} X} \longrightarrow 1$ is an effective descent morphism of $\mathcal{D} / \mathcal{D}^{*} X$. By the proof of our first lemma (Lemma 3.1) we see that $L_{\mathcal{D}^{*} X} \Sigma_{\Delta_{X}} \dashv \Delta_{X}^{*} R_{\mathcal{D}^{*} X}$ is over $\mathcal{C} / X$ and so by 1 . we known that $\Delta_{X}^{*} R_{\mathcal{D}^{*} X}\left(W_{\mathcal{D}^{*} X}\right)$ is an effective descent morphism of $\mathcal{C}$. But $W_{\mathcal{D}^{*} X}=\left(\mathcal{D}^{*} X\right)^{*} W$ and, Lemma 3.1, $R$ factors as $\Delta_{X}^{*} R_{\mathcal{D}^{*} X}\left(\mathcal{D}^{*} X\right)^{*}$ and so $R W$ is an effective descent morphism as required.

\section{Groupoids}

We now recall some facts about internal groupoids, $\mathbb{G}$ in $\mathcal{C}$, and find that they provide a plentify supply of effective descent morphisms and further that the Frobenius reciprocity and monadicity conditions, in combination, can be used to characterise when an adjunction is a connected components adjunction for an internal groupoid.

7.1. Definition. A groupoid internal to a cartesian category, $\mathcal{C}$, consists of the data

$$
\mathbb{G}=\left(G_{1} \underset{d_{1}}{\stackrel{d_{0}}{\longrightarrow}} G_{0}, m: G_{1} \times_{G_{0}} G_{1} \longrightarrow G_{1}, e: G_{0} \longrightarrow G_{1}, i: G_{1} \longrightarrow G_{1}\right)
$$

subject to the usual identities; the object of objects is $G_{0}$ and object of morphisms $G_{1} ; d_{0}$ is the domain map etc. The domain of $m$ consists of pairs $\left(g_{1}, g_{2}\right)$ such that $d_{0} g_{1}=d_{1} g_{2}$.

Example 7.1. (i) If $X$ is an object of $\mathcal{C}$ then there is the trivial groupoid $\mathbb{X}=$ $\left(X \underset{I d_{X}}{\stackrel{I d_{X}}{\longrightarrow}} X, \ldots\right)$ associated with it.

(ii) Another easy but key example of a groupoid is $\left(X \times X \underset{\pi_{2}}{\stackrel{\pi_{1}}{\longrightarrow}} X, \pi_{13}: X \times\right.$ $\left.X \times X \longrightarrow X \times X, \Delta_{X}: X \longrightarrow X \times X, \tau: X \times X \longrightarrow X \times X\right)$ for any object $X$ of $\mathcal{C}$, where $\tau$ is the twist isomorphism.

A $\mathbb{G}$-object consists of $\left(X_{f}, a: \Sigma_{d_{1}} d_{0}^{*} X_{f} \longrightarrow X_{f}\right)$ where $f: X \longrightarrow G_{0}$ and $a$ is a morphism over $G_{0}$ that satisfies the usual unit and associative identities (the domain of $\Sigma_{d_{1}} d_{0}^{*} X_{f}$ is $G_{1} \times_{G_{0}} X$ ). The map $a: G_{1} \times_{G_{0}} X \longrightarrow X$ is the $\mathbb{G}$-object's structure map. A $\mathbb{G}$-homomorphism between two $\mathbb{G}$-objects $\left(Y_{g}, b\right)$ and $\left(X_{f}, a\right)$ consists of a map $h: Y_{g} \longrightarrow X_{f}$ such that $a \Sigma_{d_{1}} d_{0}^{*}(h)=h b$. This defines the category $[\mathbb{G}, \mathcal{C}]$ of $\mathbb{G}$-objects. For example, for any object $X$ of $\mathcal{C},[\mathbb{X}, \mathcal{C}]$ is isomorphic to the slice category $\mathcal{C} / X$. As the inverse of a groupoid determines an isomorphism from $\mathbb{G}$ to $\mathbb{G}^{o p}$, we always have an isomorphism $[\mathbb{G}, \mathcal{C}] \cong\left[\mathbb{G}^{o p}, \mathcal{C}\right]$. 
The data for a groupoid $\mathbb{G}$ can be used to define a monad $\mathbb{T}_{\mathbb{G}}$ on $\mathcal{C} / G_{0}$; its functor part is $\Sigma_{d_{1}} d_{0}^{*}$ and the category of $\mathbb{G}$-objects is the same thing as the category of algebras for this monad. The counit of the associated adjunction $\mathbb{T}_{\mathbb{G}} \dashv U_{\mathbb{G}}$ : $\mathcal{C} / G_{0} \longleftrightarrow[\mathbb{G}, \mathcal{C}]$, at $\left(X_{f}, a\right)$, is $a: G_{1} \times_{G_{0}} X \longrightarrow X$.

Lemma 7.2. For any internal groupoid $\mathbb{G}, \mathbb{T}_{\mathbb{G}} \dashv U_{\mathbb{G}}: \mathcal{C} / G_{0} \rightleftarrows[\mathbb{G}, \mathcal{C}]$ is stably Frobenius.

Proof. As $U_{\mathbb{G}}$ is monadic, pullbacks in the category of $\mathbb{G}$-objects are created in $\mathcal{C} / G_{0}$. If $X$ is an object over $G_{0}$ and we are given a $\mathbb{G}$-homomorphism $k:\left(Y_{g}, b\right) \longrightarrow\left(Z_{h}, c\right)$ and a morphism $f: X \longrightarrow Z$ over $G_{0}$ then we need to show that

$$
\begin{aligned}
G_{1} \times_{G_{0}}\left(X \times_{Z} Y\right) & \longrightarrow \quad\left(G_{1} \times_{G_{0}} X\right) \times_{Z} Y \\
(g, x, y) & \mapsto \quad(g, x, b(g, y))
\end{aligned}
$$

is an isomorphism. But this is clear as $(g, x, y) \mapsto\left(g, x, b\left(g^{-1}, y\right)\right)$ can be seen to be the inverse (it is well defined as $k$ is a $\mathbb{G}$-homomorphism).

In the context of cartesian categories we are using element notation $(x, y, g$ etc) only as short hand to define morphisms.

Given the Lemma, for each $\mathbb{G}$-object $\left(X_{f}, a\right)$, there is a natural isomorphism

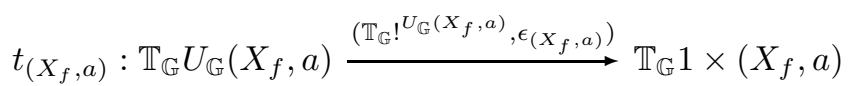

where $\epsilon$ is the conunit of $\mathbb{T}_{\mathbb{G}} \dashv U_{\mathbb{G}}$. Using this explicit description it is clear that the diagrams

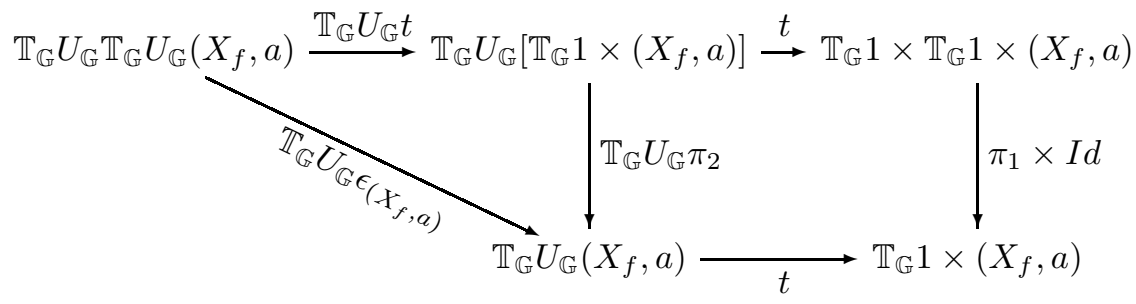

and

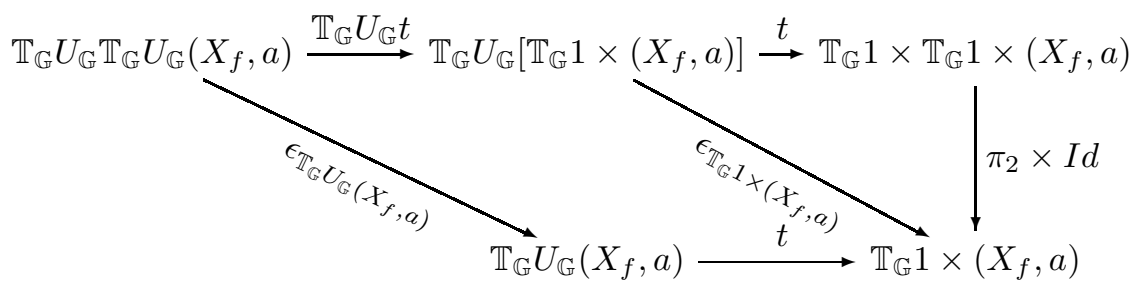

both commute by naturality of $\epsilon$ where the horizontal $t$ s are all examples of the isomorphism just described.

7.2. Connected components. There is functor $\mathbb{G}^{*}$ from $\mathcal{C}$ to $[\mathbb{G}, \mathcal{C}]$; it sends any object $X$ to $X_{G_{0}}$ with its trivial action (i.e. $\left.d_{1} \times I d_{X}: G_{1} \times X \longrightarrow G_{0} \times X\right)$. If $\mathbb{G}^{*}: \mathcal{C} \longrightarrow[\mathbb{G}, \mathcal{C}]$ has a left adjoint adjoint then the left adjoint is written $\Sigma_{\mathbb{G}}$; it is the usual connected components functor, but we have chosen to extend the pullback adjunction notation to this case. (This is not unreasonable since in the case that $\mathbb{G}$ is the groupoid internal to $\mathcal{C} / Y$ determined by $f: X \longrightarrow Y$, then $\Sigma_{\mathbb{G}} \dashv \mathbb{G}^{*}$ is the adjunction $\Sigma_{f} \dashv f^{*}$ up to isomorphism.) This left adjoint, if it 
exists, must send any $\left(X_{f}, a\right)$ to the coequalizer $X \stackrel{n}{\longrightarrow} \Sigma_{\mathbb{G}}\left(X_{f}, a\right)$ of $a$ and $\pi_{2}$. The unit of $\Sigma_{\mathbb{G}} \dashv \mathbb{G}^{*}$ at $\left(X_{f}, a\right)$ is $X \stackrel{(f, n)}{\longrightarrow} G_{0} \times \Sigma_{\mathbb{G}}\left(X_{f}, a\right)$. Connected component adjunctions do not always exist.

If $\mathbb{G}$ has a connected components adjunction then $\Sigma_{\mathbb{G}} \mathbb{T}_{\mathbb{G}}\left(X_{f}\right) \cong X$ for any object $X_{f}$ of $\mathcal{C} / G_{0}$; i.e. $\Sigma_{\mathbb{G}} \mathbb{T}_{\mathbb{G}} \cong \Sigma_{G_{0}}$. To see this, note that the action map on $\mathbb{T}_{\mathbb{G}} X_{f}$ is $\left(g_{1},\left(g_{2}, x\right)\right) \mapsto\left(g_{1} g_{2}, x\right)$ and $\pi_{2}: G_{1} \times_{G_{0}} X \longrightarrow X$ is the coequalizer, split using the identity on $\mathbb{G}$, of this action and $\left(g_{1},\left(g_{2}, x\right)\right) \mapsto\left(g_{2}, x\right)$. From this it is clear that $\Sigma_{\mathbb{G}} \epsilon_{\mathbb{T}_{\mathbb{G}} X_{f}}$ is canonically isomorphic to $\pi_{2}: G_{1} \times_{G_{0}} X \longrightarrow X$. We also note in passing that therefore $\Sigma_{\mathbb{G}}\left(\left(G_{1}\right)_{d_{1}}, m\right)$ is isomorphic to $G_{0}$ as $\left(\left(G_{1}\right)_{d_{1}}, m\right)=\mathbb{T}_{\mathbb{G}} 1$.

Now for any $\mathbb{G}$-object $\left(X_{f}, a\right), U_{\mathbb{G}} \epsilon_{\left(X_{f}, a\right)}$ is the action map $a: G_{1} \times_{G_{0}} X \longrightarrow X$ and we have just seen that $\Sigma_{\mathbb{G}} \epsilon_{\mathbb{T}_{G} U_{G}\left(X_{f}, a\right)}$ is the projection $\pi_{2}: G_{1} \times_{G_{0}} X \longrightarrow X$. Combining these observations with the diagrams noted after Lemma 7.2 we see that if $\mathbb{G}$ has a connected components adjunction then for any $\mathbb{G}$-object $\left(X_{f}, a\right)$ there are canonical horizontal isomorphisms such that both diagrams in

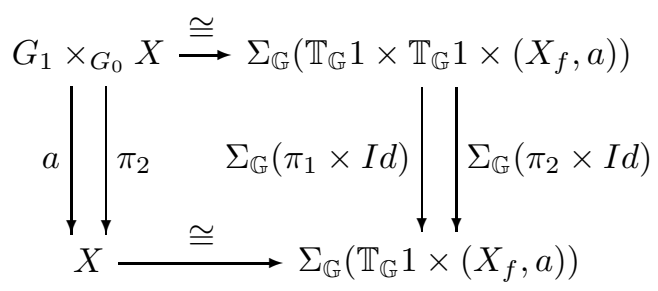

commute. The connected components functor in conjunction with the monad associated with a groupoid can be used to recover information about any $\mathbb{G}$-object. These observations will be exploited later.

The following proposition is new and provides characterisations of when an adjunction is a connected components adjunction.

Proposition 7.3. Let $\Sigma_{\mathcal{D}} \dashv \mathcal{D}^{*}: \mathcal{D} \rightleftarrows \mathcal{C}$ be an ajunction. The following are equivalent:

1. There exists an internal groupoid $\mathbb{G}$ in $\mathcal{C}$ and an equivalence $\Theta: \mathcal{D} \stackrel{\simeq}{\longrightarrow}[\mathbb{G}, \mathcal{C}]$ such that $\Theta \mathcal{D}^{*} \cong \mathbb{G}^{*}$.

2. There exists an object $G_{0}$ and a stably Frobenius adjunction $\mathbb{T} \dashv U: \mathcal{C} / G_{0} \rightleftarrows \mathcal{D}$ which is over $\mathcal{C}$ and is such that $U$ is monadic.

3. There exists an object $G_{0}$ and an adjunction $\mathbb{T} \dashv U: \mathcal{C} / G_{0} \rightleftarrows \mathcal{D}$, Frobenius at $\mathcal{D}^{*} G_{0}$, which is over $\mathcal{C}$ and is such that $U$ is monadic.

4. There exists an object $W$ of $\mathcal{D}$ such that $!^{W}: W \longrightarrow 1$ is an effective descent morphism and $\left(\Sigma_{\mathcal{D}}\right)_{W}: \mathcal{D} / W \longrightarrow \mathcal{C} / \Sigma_{\mathcal{D}} W$ is an equivalence of categories.

Proof. 1. implies 2. is essentially Lemma 7.2

2 . implies 3. is clear as 3 . is a weakening of 2 ., requiring less of the adjunction $\mathbb{T} \dashv U$.

For 3. implies 4. apply part 2. of Lemma 4.2 take $W=\mathbb{T} 1$. Because $U$ is monadic $!^{W}: W \longrightarrow 1$ is an effective descent morphism.

For 4. implies 1., define $G_{0}=\Sigma_{\mathcal{D}} W, G_{1}=\Sigma_{\mathcal{D}}(W \times W), d_{0}=\Sigma_{\mathcal{D}}\left(\pi_{2}\right)$ and $d_{1}=\Sigma_{\mathcal{D}}\left(\pi_{1}\right)$. Define $e: G_{0} \longrightarrow G_{1}$ as $\Sigma_{\mathcal{D}}\left(\Delta_{W}\right)$. For the multiplication, as $\pi_{13}: W \times W \times W \longrightarrow W$ is the product, relative to $\mathcal{D} / W$, of $\pi_{2}: W \times W \longrightarrow W$ and $\pi_{1}: W \times W \longrightarrow W$ and as $\left(\Sigma_{\mathcal{D}}\right)_{W}$ preserves products (it is an equivalence by assumption) we know that the canonical map $\Sigma_{\mathcal{D}}\left(\pi_{12}, \pi_{23}\right): \Sigma_{\mathcal{D}}(W \times W \times$ 
$W) \longrightarrow \Sigma_{\mathcal{D}}(W \times W) \times \Sigma_{\mathcal{D}}(W) \Sigma_{\mathcal{D}}(W \times W)$ is an isomorphism. Define $m: G_{1} \times_{G_{0}}$ $G_{1} \longrightarrow G_{1}$ to be $\Sigma_{\mathcal{D}}\left(\pi_{13}\right) \Sigma_{\mathcal{D}}\left(\pi_{12}, \pi_{23}\right)^{-1}$. Define $i: G_{1} \longrightarrow G_{1}$ as $\Sigma_{\mathcal{D}}(\tau)$.

Given that $\left(\Sigma_{\mathcal{D}}\right)_{W}$ preserves products and the various pullbacks involved are products in $\mathcal{D} / W$ it is easy to see that a groupoid $\mathbb{G}$ in $\mathcal{C}$ has been defined because of our earlier example (7.1) (ii) which shows that $\left(W \times W \rightrightarrows W, \pi_{13}, \ldots\right)$ is always a groupoid.

Since ! : $W \longrightarrow 1$ is an effective descent morphism we can identify $\mathcal{D}$ with the category of algebras of the monad on $\mathcal{D} / W$ induced by the adjunction $\Sigma_{W} \dashv W^{*}$. Now say $h: V \longrightarrow W$ is an object of $\mathcal{D} / W$ and write $X_{f}$ for its image under $\left(\Sigma_{\mathcal{D}}\right)_{W}$. As $W \times V \stackrel{h \pi_{2}}{\longrightarrow} W$ is the product of $\pi_{2}: W \times W \longrightarrow W$ and $V_{h}$ in $\mathcal{D} / W$ and $\left(\Sigma_{\mathcal{D}}\right)_{W}$ preserves products we know that $\Sigma_{\mathcal{D}}(V \times W) \cong \Sigma_{\mathcal{D}}(W \times W) \times \Sigma_{\mathcal{D}} W$ $\Sigma_{\mathcal{D}} V=G_{1} \times G_{0} X$, the pullback of $f$ along $d_{0}$. Further, $W^{*} \Sigma_{W} V_{h}$ is the projection $\pi_{1}: W \times V \longrightarrow W$ which can be written $W_{W} \times V_{h} \stackrel{\pi_{1}}{\longrightarrow} W_{W} \stackrel{!^{W_{W}}}{\longrightarrow} 1_{\mathcal{D} / W}$ and therefore maps to $\Sigma_{d_{1}} d_{0}^{*} X_{f}$ under $\left(\Sigma_{\mathcal{D}}\right)_{W}$ as $!^{W_{W}}$ is $\pi_{1}: W \times W \longrightarrow W$. It can then be verified that a map $a: W \times V \longrightarrow V$ over $W$ is an algebra of the monad induced by $\Sigma_{W} \dashv W^{*}$ if and only if its image under $\Sigma_{\mathcal{D}}$ is a $\mathbb{G}$-object and similarly for homomorphisms.

The characterisations of this proposition will be used extensively in what follows essentially as they give us results about $\mathbb{G}$-objects without having to argue in detail about various pullbacks. As an initial example, because $W$ is taken to be $\mathbb{T} 1$ in the proof of 2. implies 4.:

Lemma 7.4. For any groupoid $\mathbb{G}$ with a connected components adjunction, ! : $\mathbb{T}_{\mathbb{G}} 1 \longrightarrow 1$ is an effective descent morphism (i.e. ! : $\left(\left(G_{1}\right)_{d_{1}}, m\right) \longrightarrow 1$ is an effective descent morphism).

As another initial example of the usefulness of the proposition:

Lemma 7.5. Let $\mathbb{G}$ be a groupoid in $\mathcal{C}$ with a connected components functor. If $\left(X_{f}, a\right)$ is a $\mathbb{G}$-object then there exists a groupoid $\mathbb{X}_{f, a}$ in $\mathcal{C}$ and an equivalence $\left[\mathbb{X}_{f, a}, \mathcal{C}\right] \simeq[\mathbb{G}, \mathcal{C}] /\left(X_{f}, a\right)$ over $\mathcal{C}$.

Proof. Consider 2 of the Proposition; both the conditions on the adjunction are stable under slicing.

The groupoid that is obtained in this process is the familiar one: $\left(G_{1} \times G_{0}\right.$ $\left.X \underset{a}{\stackrel{\pi_{2}}{\rightleftarrows}} X, \ldots\right)$. The equivalence established can be summarised as follows: if $\left(Y_{g}, b\right)$ is a $\mathbb{X}_{f, a}$-object then the domain of its structure map is the pullback of $g: Y \longrightarrow X$ along $\pi_{2}: G_{1} \times_{G_{0}} X \longrightarrow X$; i.e. $G_{1} \times_{G_{0}} Y$ and so the structure map $b$ of $\left(Y_{g}, b\right)$ can also be used to provide a structure map for $Y_{f g}$, turning it into a G-object in such a way that $g$ is $\mathbb{G}$-homomorphism.

\section{Hilsum-SKANDALIS MAPS}

Given two internal groupoids $\mathbb{H}$ and $\mathbb{G}$, a Hilsum-Skandalis map from $\mathbb{H}$ to $\mathbb{G}$ will be defined as a particular $\mathbb{H} \times \mathbb{G}$-object. It is easy to verify that the data for a $\mathbb{H} \times \mathbb{G}$ object can be considered to be a triple $\left(P \stackrel{(p, q)}{\longrightarrow} H_{0} \times G_{0}, c: H_{1} \times H_{0} P \longrightarrow P, d\right.$ : $G_{1} \times_{G_{0}} P \longrightarrow P$ ) such that

(i) $\left(P_{p}, c\right)$ is an $\mathbb{H}$-object, 
(ii) $\left(P_{q}, d\right)$ is a $\mathbb{G}$-object,

(iii) $p$ is $d$ invariant; i.e. $p \pi_{2}=p d$

(iv) $q$ is $c$ invariant; i.e. $q \pi_{2}=q c$

(v) $c$ and $d$ commute; i.e. $d\left(I d_{G_{1}} \times c\right)=c\left(I d_{H_{1}} \times d\right)\left(\tau \times I d_{P}\right)$ where $\tau$ : $G_{1} \times H_{1} \longrightarrow H_{1} \times G_{1}$ is the twist isomorphism.

Similarly a morphism can be seen to be a $\mathbb{H} \times \mathbb{G}$-homomorphism if and only if it is both a $\mathbb{H}$-homomorphism and a $\mathbb{G}$-homomorphism.

Definition 8.1. Given two internal groupoids $\mathbb{H}$ and $\mathbb{G}$ in $\mathcal{C}$ a Hilsum-Skandalis map from $\mathbb{H}$ to $\mathbb{G}$ is a $\mathbb{H} \times \mathbb{G}$-object $\mathfrak{P}=\left(P \stackrel{(p, q)}{\longrightarrow} H_{0} \times G_{0}, c: H_{1} \times{ }_{H_{0}} P \longrightarrow P, d:\right.$ $\left.G_{1} \times G_{0} P \longrightarrow P\right)$ such that

(i) $\left(d, \pi_{2}\right): G_{1} \times_{G_{0}} P \longrightarrow P \times_{H_{0}} P$ is an isomorphism, and

(ii) $p: P \longrightarrow H_{0}$ is an effective descent morphism.

$A$ morphism between Hilsum-Skandalis maps $\mathfrak{P} \longrightarrow \mathfrak{P}^{\prime}$ is a map $\theta: P \longrightarrow P^{\prime}$ that is both a $\mathbb{H}$-homomorphism and $a \mathbb{G}$-homomorphism; i.e. it is just a morphism of $[\mathbb{H} \times \mathbb{G}, \mathcal{C}]$.

Note that the inverse of $\left(d, \pi_{2}\right)$, if it exists, must be of the form $\left(\psi, \pi_{2}\right)$ for some $\psi: P \times_{H_{0}} P \longrightarrow G_{1}$.

If $X$ is an object of $\mathcal{C}$ then a principal $\mathbb{G}$-bundle over $X$ is a Hilsum-Skandalis morphism from $\mathbb{X}$ to $\mathbb{G}$. This definition recovers the usual meaning, with 'effective descent morphism' playing the role of open surjection. So, intuitively, HilsumSkandalis maps are generalisations of principal bundles; for principal bundles the 'domain' is determined by an object and the 'codomain' is a groupoid, but for Hilsum-Skandalis maps both the domain and codomain are groupoids. Consult Mr96 for background material on Hilsum-Skandalis maps. The definition is usually phrased in terms of topological groupoids, where the requirement (ii) on $p$ is for an open surjection. In the context of cartesian categories therefore we are not strictly just generalising the topological situation since there is no notion of 'open'. However, as we shall see, all the main results still go through and it is easy to specialise to the open case if required. There are two other minor differences to the standard definition:

(i) because $\mathbb{G}$ is homeomorphic to $\mathbb{G}^{o p}$ we are not distinguishing the two actions in terms of their handedness.

(ii) we are not isolating a map up to an equivalence relation determined by morphisms between Hilsum-Skandalis maps. We will keep track of these 2-cells, though, as is well known and we will now show, they are all isomorphisms:

Proposition 8.2. Let $\left(P_{(p, q)}, c, d\right)$ and $\left(P_{\left(p^{\prime}, q^{\prime}\right)}^{\prime}, c^{\prime}, d^{\prime}\right)$ be two Hilsum-Skandalis maps (from $\mathbb{H}$ to $\mathbb{G})$. (a) If $\theta:\left(P_{q}, d\right) \longrightarrow\left(P_{q^{\prime}}^{\prime}, d^{\prime}\right)$ is a $\mathbb{G}$-homomorphism then $\theta$ is an isomorphism in $[\mathbb{G}, \mathcal{C}]$. (b) If, further, $\theta$ is a morphism between HilsumSkandalis maps, i.e. it is also an $\mathbb{H}$-homomorphism, then it is an isomorphism in $[\mathbb{H} \times \mathbb{G}, \mathcal{C}]$.

Proof. (a) It is sufficient to prove that $\theta$ is an isomorphism in $\mathcal{C}([\mathbb{G}, \mathcal{C}]$ is a category of algebras over $\left.\mathcal{C} / G_{0}\right)$. Because $p: P \longrightarrow H_{0}$ is an effective descent morphism it is sufficient to prove $\theta \times I d_{P}: P \times_{H_{0}} P \longrightarrow P^{\prime} \times{ }_{H_{0}} P$ is an isomorphism because $p^{*}$ reflects isomorphisms. Its inverse is given by

$$
P^{\prime} \times_{H_{0}} P \stackrel{\left(\pi_{1}, \theta \pi_{2}, \pi_{2}\right)}{\longrightarrow} P^{\prime} \times_{H_{0}} P^{\prime} \times_{H_{0}} P \stackrel{\psi^{\prime} \times I d_{P}}{\longrightarrow} G_{1} \times_{G_{0}} P \stackrel{\left(d, \pi_{2}\right)}{\longrightarrow} P \times_{H_{0}} P .
$$


CHRISTOPHER TOWNSEND

To see this use the fact that $\psi$ must factor as $\psi^{\prime}(\theta \times \theta)$ as $\theta$ is a $\mathbb{G}$-homomorphism and $\pi_{1}: P^{\prime} \times_{H_{0}} P^{\prime} \longrightarrow P^{\prime}$ factors as $d^{\prime}\left(\psi^{\prime}, \pi_{2}\right)$.

(b) follows from (a).

The next Proposition provides a class of examples of Hilsum-Skandalis maps. We will see later that with a further restriction on the groupoids, all Hilsum-Skandalis maps arise in this way.

Proposition 8.3. Let $L \dashv R:[\mathbb{H}, \mathcal{C}] \rightleftarrows[\mathbb{G}, \mathcal{C}]$ be a stably Frobenius adjunction over $\mathcal{C}$, where both $\mathbb{G}$ and $\mathbb{H}$ have connected component adjunctions. If $\left(P_{p}, c\right.$ : $\left.H_{1} \times{ }_{H_{0}} P \longrightarrow P\right)$ is defined as $R \mathbb{T}_{\mathbb{G}} 1$ and $\left(Q_{q}, d: G_{1} \times_{G_{0}} Q \longrightarrow Q\right)$ as $L \mathbb{T}_{\mathbb{H}} 1$, then there exists an isomorphism $\psi: P \stackrel{\cong}{\longrightarrow} Q$ and $\left(P_{(p, q \psi)}, c, \psi^{-1} d\left(I_{G_{0}} \times \psi\right)\right)$, is a Hilsum-Skandalis map from $\mathbb{H}$ to $\mathbb{G}$.

Proof. By Lemma 7.4 we know that ! : $\mathbb{T}_{\mathbb{G}} 1 \longrightarrow 1$ is an effective descent morphism. Then by part 2. of Lemma 6.1 applied to $L \mathbb{T}_{\mathbb{H}} \dashv U_{\mathbb{H}} R$ we know that $p: P \longrightarrow H_{0}$ is an effective descent morphism.

Since $L \dashv R$ is Frobenius and over $\mathcal{C}$ there are isomorphisms

$$
\Sigma_{\mathbb{H}}\left[\left(Y_{g}, b\right) \times R\left(X_{f}, a\right)\right] \stackrel{\cong}{\longrightarrow} \Sigma_{\mathbb{G}}\left[\left(X_{f}, a\right) \times L\left(Y_{g}, b\right)\right]
$$

natural in $\mathbb{G}$-objects $\left(X_{f}, a\right)$ and $\mathbb{H}$-objects $\left(Y_{g}, b\right)$. By naturality:

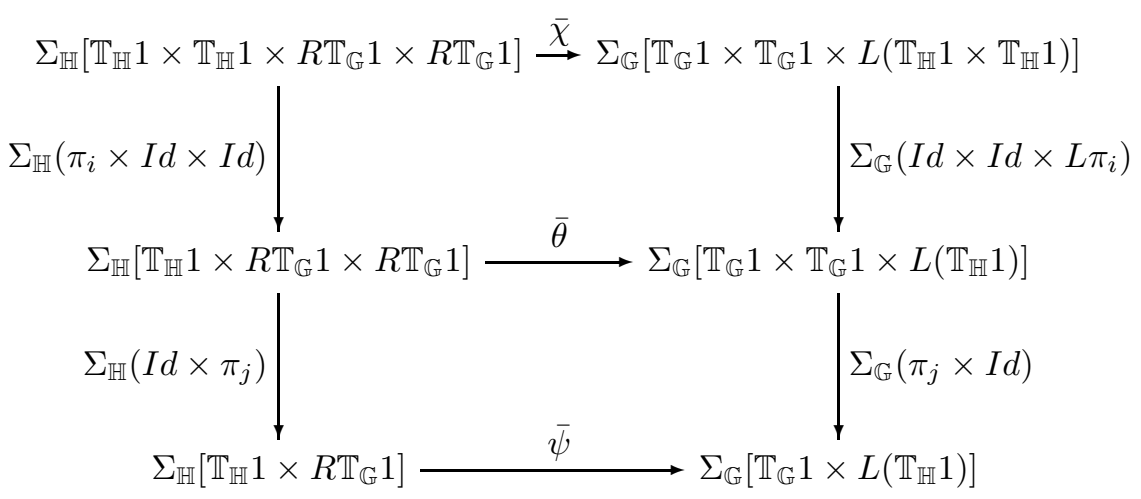

and

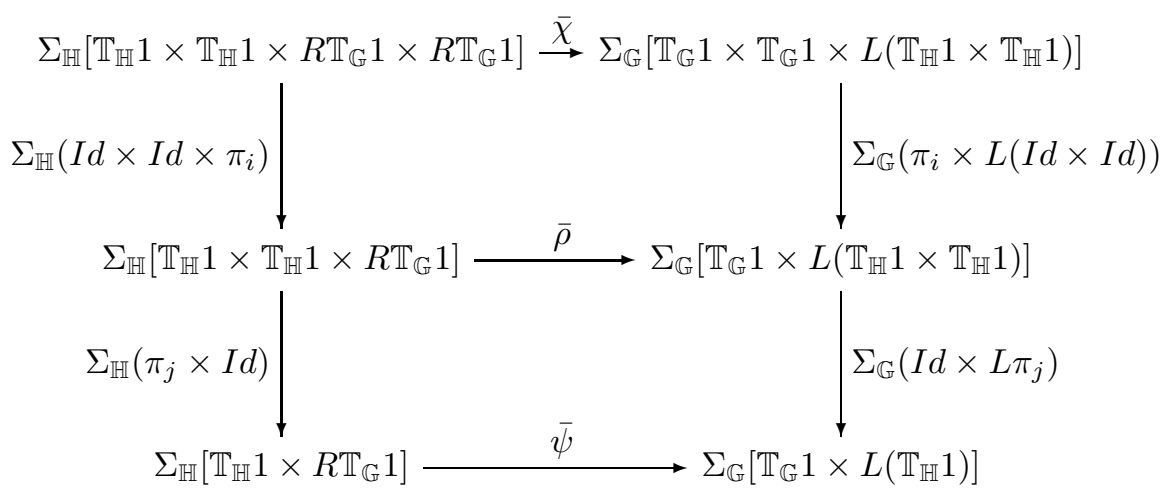


commute for $i, j=1,2$ in both diagrams. But, applying the diagram before Proposition 7.3 we then have that both squares in

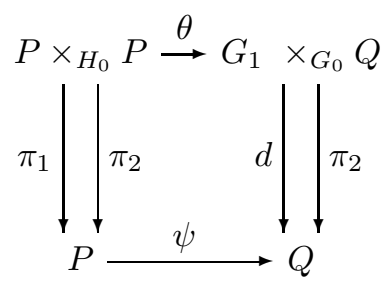

commute by considering $j=1,2$ in the bottom square of the first diagram and noting the natural isomorphisms $\Sigma_{H_{0}} U_{\mathbb{H}} \cong \Sigma_{\mathbb{H}} \mathbb{T}_{\mathbb{H}} U_{\mathbb{H}} \cong \Sigma_{\mathbb{H}}\left[\mathbb{T}_{\mathbb{H}} 1 \times(-)\right]$. Then $G_{1} \times_{G_{0}}$ $P \stackrel{\left(\star, \pi_{2}\right)}{\longrightarrow} P \times_{H_{0}} P$ has an inverse, where $\star$ is the action $d$ translated into an action on $P$ via $\psi$ (i.e. $\left.\star=\psi^{-1} d\left(I d_{G_{1}} \times \psi\right)\right)$ and $q \psi$ must be $\star$ invariant as the diagram shows that $p \psi^{-1}$ is $d$-invariant.

Write $\left(R_{r}, e\right)$ for $L\left(\mathbb{T}_{\mathbb{H}} \times \mathbb{T}_{\mathbb{H}} 1\right)$, and $p_{1}, p_{2}: R \longrightarrow Q$ for the $\mathbb{G}$-homomorphisms $L \pi_{1}$ and $L \pi_{2}$. Then by the bottom square of the second diagram we have commuting diagrams

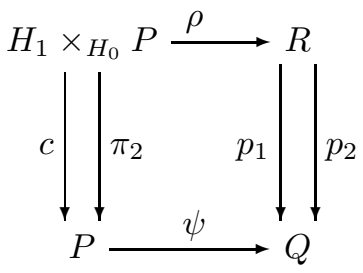

from which it is clear that $q \psi$ is $c$-invariant.

To complete this proof we must prove that the two actions $d$ and $c$ commute. Firstly note that the additional commuting squares above can be exploited to confirm that, $\omega$, defined as

$H_{1} \times_{H_{0}}\left(G_{1} \times_{G_{0}} Q\right) \stackrel{I d \times \theta^{-1}}{\longrightarrow} H_{1} \times_{H_{0}}\left(P \times_{H_{0}} P\right) \stackrel{\chi}{\longrightarrow} G_{1} \times_{G_{0}} R \stackrel{I d \times \rho^{-1}}{\longrightarrow} G_{1} \times_{G_{0}}\left(H_{1} \times_{H_{0}} P\right)$ is $\tau \times \psi$. To see this observe: $\left(I d \times \pi_{2}\right) \omega=\left(I d \times \psi^{-1}\right)\left(I d \times p_{2}\right) \chi\left(I d \times \theta^{-1}\right)=$ $\left(I d \times \psi^{-1}\right) \theta \pi_{23}\left(I d \times \theta^{-1}\right)=\left(I d \times \psi^{-1}\right) \pi_{23}$ and $\pi_{23} \omega=\rho^{-1} \pi_{2} \chi\left(I d \times \theta^{-1}\right)=$ $\rho^{-1} \rho\left(I d \times \pi_{2}\right)\left(I d \times \theta^{-1}\right)=\left(I d \times \psi^{-1}\right)\left(I d \times \pi_{2}\right)$. Combining it is clear that $\omega=\tau \times \psi$.

Finally, as $p_{1}$ is a $\mathbb{G}$-hommorphsm, $p_{1} e=d\left(I d \times p_{1}\right)$ and so $c\left(I d \times \psi^{-1}\right)(I d \times d)=$ $\left(\psi^{-1} p_{1} \rho\right)\left(I d \times \pi_{1}\right)\left(I d \times \theta^{-1}\right)=\psi^{-1} p_{1} e \chi\left(I d \times \theta^{-1}\right)=\psi^{-1} d\left(I d \times p_{1}\right) \chi\left(I d \times \theta^{-1}\right)=$ $\psi^{-1} d(I d \times \psi)(I d \times c)\left(I d \times \rho^{-1}\right) \chi\left(I d \times \theta^{-1}\right)=\psi^{-1} d(I d \times \psi)(I d \times c) \omega$.

In what follows we will use the notation $\mathfrak{P}_{L, R}$ for the Hilsum-Skandalis map corresponding to an adjunction $L \dashv R$.

\section{INTERNAL FUNCTORS}

An internal functor $\mathbb{F}: \mathbb{H} \longrightarrow \mathbb{G}$ gives rise to a functor $\mathbb{F}^{*}:[\mathbb{G}, \mathcal{C}] \longrightarrow[\mathbb{H}, \mathcal{C}]$ at the level of $\mathbb{G}$-objects. Explicitly the functor $\mathbb{F}^{*}$ sends $\left(X_{f}, a\right)$ to $\left(\pi_{1}: H_{0} \times_{G_{0}}\right.$ $\left.X \longrightarrow H_{0},\left(d_{1}^{\mathbb{H}} \pi_{1}, a\left(F_{1} \times I d_{X}\right)\right): H_{1} \times_{G_{0}} X \longrightarrow H_{0} \times_{G_{0}} X\right)$, where $H_{0} \times_{G_{0}} X$ is the pullback of $f$ along $F_{0}: H_{0} \longrightarrow G_{0}$. The functor $\mathbb{F}^{*}$ does not in general have a left adjoint. To see this consider for an internal groupoid $\mathbb{G}$ the unique internal functor $!^{\mathbb{G}}: \mathbb{G} \longrightarrow 1$; then $\left(!^{\mathbb{G}}\right)^{*}: \mathcal{C} \longrightarrow[\mathbb{G}, \mathcal{C}]$ is $\mathbb{G}^{*}$, and so it has a left adjoint 
if and only if $\mathbb{G}$ has a connected components adjunction and not every internal groupoid has a connected components adjunction.

However the following lemma provides a class of functors for which the left adjoint does exist and the resulting adjunction is stably Frobenius:

Lemma 9.1. Let $\mathbb{F}: \mathbb{H} \longrightarrow \mathbb{G}$ be an internal functor. Then $\left(I d_{\mathbb{H}}, \mathbb{F}\right)^{*}:[\mathbb{H} \times$ $\mathbb{G}, \mathcal{C}] \longrightarrow[\mathbb{H}, \mathcal{C}]$ has a left adjoint $\Sigma_{\left(I d_{\mathbb{H}}, \mathbb{F}\right)}$ such that $\Sigma_{\left(I d_{\mathbb{H}}, \mathbb{F}\right)} \dashv\left(I d_{\mathbb{H}}, \mathbb{F}\right)^{*}$ is stably Frobenius.

Proof. Let $Z=\left(Z_{(a, b)}, *_{\mathbb{H}}, *_{\mathbb{G}}\right)$ be an $\mathbb{H} \times \mathbb{G}$-object. Then observe that $\left(I d_{\mathbb{H}}, \mathbb{F}\right)^{*} Z$ is given by $E_{a e}$ where $E \stackrel{e}{\longrightarrow} Z$ is the equalizer of $F_{0} a: Z \longrightarrow H_{0} \longrightarrow G_{0}$ and $b$ : $Z \longrightarrow G_{0}$; the action is given by the factorization through $e$ of $\star:(h, i) \mapsto h F_{1} h e i$ where we are using concatenation for both $*_{\mathbb{H}}$ and $*_{\mathbb{G}}$. To ease the notation we drop the $e$ in what follows. Given an $\mathbb{H}$-object $Y=\left(Y_{p}, *_{Y}\right)$, consider the pullback of $d_{1}^{\mathbb{G}}$ along $F_{0} p$, i.e. $Y \times_{G_{0}} G_{1}=\left\{\left(y, g_{0}\right) \mid F_{0} p y=d_{1}^{\mathbb{G}} g_{0}\right\}$. Define $\Sigma_{\left(I d_{\mathbb{H}}, \mathbb{F}\right)} Y$ to be $\left(Y \times G_{0} G_{1}\right)_{p \times d_{0}^{\mathbb{G}}}$ with action $(h, g) *\left(y, g_{0}\right)=\left(h y, F_{1} h g_{0} g^{-1}\right)$; it can be checked that this is an $\mathbb{H} \times \mathbb{G}$-object and clearly a functor is defined. We now check that $\Sigma_{\left(I d_{\mathbb{H}}, \mathbb{F}\right)} \dashv\left(I d_{\mathbb{H}}, \mathbb{F}\right)^{*}$. Say $\Psi: Y \times_{G_{0}} G_{1} \longrightarrow Z$ is an $\mathbb{H} \times \mathbb{G}$-homomorphism. Then pre-composition with $\left(I d_{Y}, e^{\mathbb{G}} F_{0} p\right)$ creates a morphism that composes equally with $F_{0} a$ and $b$ as $\Psi$ is a morphism over $H_{0} \times G_{0}$. So $\Psi\left(I d_{Y}, e^{\mathbb{G}} F_{0} p\right): Y \longrightarrow Z$ factors through $E$ and the resulting map, written $\tilde{\Psi}$, is an $\mathbb{H}$-homomorphism because

$$
\begin{aligned}
h \star\left(\Psi\left(I d_{Y}, e^{\mathbb{G}} F_{0} p\right) y\right) & =h F_{1} h \Psi\left(y, e^{\mathbb{G}} F_{0} p y\right) \\
& =\Psi\left[\left(h, F_{1} h\right) *\left(y, e^{\mathbb{G}} F_{0} p y\right)\right] \\
& =\Psi\left(h y,\left(F_{1} h\right)\left(e^{\mathbb{G}} F_{0} p y\right)\left(F_{1} h\right)^{-1}\right) \\
& =\Psi\left(h, e^{\mathbb{G}} F_{0} p(h y)\right) \\
& =\Psi\left(I d_{Y}, e^{\mathbb{G}} F_{0} p\right)(h y)
\end{aligned}
$$

In the other direction, say we are given an $\mathbb{H}$-homomorphism $\psi: Y \longrightarrow E$, then define $\bar{\psi}: Y \times_{G_{0}} G_{1} \longrightarrow Z$ by $\left(y, g_{0}\right) \mapsto g_{0}^{-1} \psi y$. Note that $b \psi y=F_{0} p y$ as $\psi$ is over $H_{0}$; so $\underline{g}_{0}^{-1} \psi y$ makes sense in this definition. Because $\psi$ composes equally with $F_{0} a$ and $b, \bar{\psi}$ is over $H_{0} \times G_{0}$. But $\bar{\psi}\left(g\left(y, g_{0}\right)\right)=\bar{\psi}\left(y, g_{0} g^{-1}\right)=g g_{0}^{-1} \psi y=g \bar{\psi}\left(y, g_{0}\right)$ and $\bar{\psi}\left(h\left(y, g_{0}\right)\right)=\bar{\psi}\left(h y, F_{1} h g_{0}\right)=g_{0}^{-1}\left(F_{1} h\right)^{-1} \psi(h y)=g_{0}^{-1}\left(F_{1} h\right)^{-1}[h \star \psi(y)]=$ $g_{0}^{-1}\left(F_{1} h\right)^{-1} F_{1} h \psi y=h g_{0}^{-1} \psi y$ (recall that $*_{\mathbb{H}}$ and $*_{\mathbb{G}}$ commute) and so $\bar{\psi}$ is an $\mathbb{H} \times \mathbb{G}$-homomorphism. It is easy to see that $\widetilde{(-)}$ and $\left({ }_{-}\right)$are natural and are bijections and so $\Sigma_{\left(I d_{\mathbb{H}}, \mathbb{F}\right)} \dashv\left(I d_{\mathbb{H}}, \mathbb{F}\right)^{*}$. For the stably Frobenius claim, note that the adjoint transpose of the identity on $E$ (i.e. on $\left(I d_{\mathbb{H}}, \mathbb{F}\right)^{*} Z$ ) is the map $E \times_{G_{0}} G_{1} \longrightarrow Z$ given by $(z, g) \mapsto g^{-1} z$; and so this gives an explicit description of the counit of the adjunction $\Sigma_{\left(I d_{\mathbb{H}}, \mathbb{F}\right)} \dashv\left(I d_{\mathbb{H}}, \mathbb{F}\right)^{*}$. So to prove the stably Frobenius claim we must verify that for any $\mathbb{H} \times \mathbb{G}$-homomorphisms $\Psi: Z \longrightarrow Z^{\prime}$ and $\Phi: Y \times_{G_{0}} G_{1} \longrightarrow Z^{\prime}$ that

$$
\begin{aligned}
&\left(Y \times_{E^{\prime}} E\right) \times_{G_{0}} G_{1} \longrightarrow \\
&\left(y, z, g_{0}\right) \mapsto \quad\left(Y \times_{G_{0}} G_{1}\right) \times_{Z^{\prime}} Z \\
&\left(y, g_{0}, g_{0}^{-1} z\right)
\end{aligned}
$$

is an isomorphism (where $\left.E^{\prime}=\left(I d_{\mathbb{H}}, \mathbb{F}\right)^{*} Z^{\prime}\right)$. This can easily be established by verifying that $\left(y, g_{0}, z\right) \mapsto\left(y, g_{0} z, g_{0}\right)$ is well defined (i.e. factors through $\left(Y \times_{E^{\prime}}\right.$ $\left.E) \times{ }_{G_{0}} G_{1}\right)$. The verification is straightforward given the following two observations: 
(i) if $\left(y, g_{0}, z\right)$ is an 'element' of $\left(Y \times_{G_{0}} G_{1}\right) \times{ }_{Z^{\prime}} Z$ then $b z$ is $d_{0}^{\mathbb{G}} g_{0}$ and so $b g_{0} z=$ $d_{1}^{\mathbb{G}} g_{0}=F_{0} p y=F_{0} a z=F_{0} a g_{0} z$, the last equality because $a$ is $\mathbb{G}$-equivariant, and so $g_{0} z$ factors through $E$, and (ii) $\Psi g_{0} z=g_{0} \Psi z=g_{0} \Phi\left(y, g_{0}\right)=\Phi\left(y, g_{0} g_{0}^{-1}\right)=$ $\Phi\left(y, e^{\mathbb{G}} F_{0} p y\right)=\tilde{\Phi} y$ and so $\left(y, g_{0} z\right)$ factors through $\left(Y \times_{E^{\prime}} E\right)$.

Definition 9.2. An internal functor $\mathbb{F}: \mathbb{H} \longrightarrow \mathbb{G}$ between two internal groupoids is (i) fully faithful provided the canonical morphism from $H_{1}$ to the pullback of $\left(d_{0}^{\mathbb{G}}, d_{1}^{\mathbb{G}}\right): G_{1} \longrightarrow G_{0} \times G_{0}$ along $H_{0} \times H_{0} \stackrel{F_{0} \times F_{0}}{\longrightarrow} G_{0} \times G_{0}$ is an isomorphism,

(ii) essentially surjective if $H_{0} \times G_{0} G_{1} \stackrel{d_{0}^{\mathbb{G}} \pi_{2}}{\longrightarrow} G_{0}$ is an effective descent morphism (where the domain $H_{0} \times{ }_{G_{0}} G_{1}$ is the pullback of $d_{1}^{\mathbb{G}}$ along $F_{0}: H_{0} \longrightarrow G_{0}$ ); and, (iii) an essential equivalence if it is both fully faithful and essentially surjective.

Example 9.3. A morphism $f: Y \longrightarrow X$, treated as an internal functor from $\mathbb{Y}$ to $\mathbb{X}$, is an essential equivalence if and only if it is an isomorphism. Any effective descent morphism is the coequalizer of its kernel pair; but the kernel pair must be the identity $(X \longrightarrow X)$ if the internal functor is fully faithful.

Hilsum-Skandalis maps can be characterised in terms of essential equivalences:

Proposition 9.4. $A \mathbb{H} \times \mathbb{G}$-object $\mathfrak{P}=\left(P \stackrel{(p, q)}{\longrightarrow} H_{0} \times G_{0}, c: H_{1} \times H_{0} P \longrightarrow P, d\right.$ : $\left.G_{1} \times G_{0} P \longrightarrow P\right)$ is a Hilsum-Skandalis map if and only if the internal projection functor $\mathfrak{p}: \mathbb{P} \longrightarrow \mathbb{H}$ is an essential equivalence, where $\mathbb{P}$ is the internal groupoid corresponding to $\mathfrak{P}$ (Lemma 7.5).

Proof. The internal projection functor is given by $p: P \longrightarrow H_{0}$ (on objects) and $\pi_{1}: H_{1} \times H_{0} G_{1} \times_{G_{0}} P \longrightarrow H_{1}$ (on morphisms). The pullback of $H_{1} \stackrel{\left(d_{0}^{\mathbb{H}}, d_{1}^{\mathbb{H}}\right)}{\longrightarrow} H_{0} \times H_{0}$ along $p \times p$ is $H_{1} \times{ }_{H_{0}} \times H_{0}(P \times P)=\left\{\left(h, x_{1}, x_{2}\right) \mid p x_{1}=d_{0}^{\mathbb{H}} h, p x_{2}=d_{1}^{\mathbb{H}} h\right\}$ which is isomorphic to $H_{1} \times{ }_{H_{0}} P \times_{H_{0}} P=\left\{(h, y, z) \mid p y=p z=d_{0}^{\mathbb{H}} h\right\} \operatorname{using}\left(h, x_{1}, x_{2}\right) \mapsto$ $\left(h, x_{1}, h^{-1} x_{2}\right)$ and $(h, y, z) \mapsto(h, y, h z)$. Under this isomorphism the canonical map from the morphism object of $\mathbb{P}$ (i.e. from $H_{1} \times{ }_{H_{O}} G_{1} \times{ }_{G_{0}} P$ ) to $H_{1} \times{ }_{H_{0}} P \times_{H_{0}} P$ is $I d_{H_{1}} \times\left(\pi_{2}, e\right): H_{1} \times H_{0} G_{1} \times_{G_{0}} P \longrightarrow H_{1} \times_{H_{0}} P \times_{H_{0}} P$ and this last is an isomorphism if and only if $G_{1} \times G_{0} P \stackrel{\left(\pi_{2}, e\right)}{\longrightarrow} P \times_{H_{0}} P$ is an isomorphism as $H_{1} \times H_{0}(-)$ reflects isomorphisms (since $d_{0}^{\mathbb{H}}$ is a split epimorphism and so is an effective descent morphism). So the internal projection functor is fully faithful if and only if condition (i) in the definition of Hilsum-Skandalis morphism is true.

The projection functor will be an essential surjection if and only if $P \times H_{0}$ $H_{1} \stackrel{\pi_{2}}{\longrightarrow} H_{1} \stackrel{d_{0}^{\mathbb{E}}}{\longrightarrow} H_{0}$ is an effective descent morphism where the domain is the pullback of $p$ along $d_{1}^{\mathbb{H}}$. But as $\left(P_{p}, c\right)$ is an $\mathbb{H}$-object, this morphism factors as $p \tilde{c}$ where $\tilde{c}(x, h)=h^{-1} x$. Since $\tilde{c}$ is a split epimorphism we know (see the introductory paragraph of the section on Effective Descent) that $p \tilde{c}$ is an effective descent morphism if and only if $p$ is.

Given a $\mathbb{H} \times \mathbb{G}$-object $\mathfrak{P}$ there is also an internal projection functor $\mathfrak{q}: \mathbb{P} \longrightarrow \mathbb{G}$. So, for any Hilsum-Skadalis map $\mathfrak{P}$, we have a span $\mathbb{H} \stackrel{\mathfrak{p}}{\longleftarrow} \mathbb{P} \stackrel{\mathfrak{q}}{\longrightarrow} \mathbb{G}$ where $\mathfrak{p}$ is an essential equivalence.

\section{Stably Frobenius Groupoids}

We will also be focusing on certain stably Frobenius adjunctions as a matter of definition: 
Definition 10.1. A groupoid $\mathbb{G}$ internal to a cartesian category $\mathcal{C}$ is stably Frobenius provided the functor $\mathbb{G}^{*}: \mathcal{C} \longrightarrow[\mathbb{G}, \mathcal{C}]$ has a left adjoint and the resulting adjunction is stably Frobenius.

If $\mathcal{C}$ has coequalizers then $\Sigma_{\mathbb{G}}$ always exists and all groupoids are stably Frobenius provided that coequalizers are pullback stable. Not all groupoids with connected component adjunctions are stably Frobenius: consider the groupoid determined by the kernel pair of a regular epimorphism that is not pullback stable. Open and proper groupoids in the category of locales provide examples of groupoids that are stably Frobenius in a cartesian category that does not have pullback stable regular epimorphisms in general. We suggest that restricting to stably Frobenius groupoids is, in an intuitive sense, the right thing to. It covers all the key cases and does not leave us figuring out which coequalizers are required of the ambient cartesian category $\mathcal{C}$. Having the connected components functor provides all the coequalizers we need, requiring the resulting connected components adjunction to be stably Frobenius allows for a number of nice results to go through, as we show with the next few propositions and the key results on Hilsum-Skandalis maps to follow. The restriction to stably Frobenius groupoids is not a serious barrier to applications; most localic groupoids are stably Frobenius and similarly for the topological case.

It can be checked that if $\mathbb{G}$ has a connected components adjunction, then it is stably Frobenius if and only for any $\mathbb{G}$-object $\left(X_{f}, a\right)$, the coequalizer that determines $\Sigma_{\mathbb{G}}\left(X_{f}, a\right)$ is pullback stable in the strong sense that for any $f: Y \longrightarrow Z$ the pullback functor $f^{*}$ preserves the coequalizer diagram that determines $\Sigma_{\mathbb{G}}\left(X_{f}, a\right)$, if that diagram is over $Z$.

We now provide a specialisation of part of Proposition 7.3. This starts our process of demonstrating how stably Frobenius groupoids are convenient by showing that once we restrict to them proving that an adjunction is a connected components adjunction becomes easier.

Proposition 10.2. Let $\Sigma_{\mathcal{D}} \dashv \mathcal{D}^{*}: \mathcal{D} \rightleftarrows \mathcal{C}$ be a stably Frobenius ajunction. The following are equivalent:

1. There exists an internal groupoid $\mathbb{G}$ in $\mathcal{C}$ and an equivalence $\Theta: \mathcal{D} \stackrel{\simeq}{\longrightarrow}[\mathbb{G}, \mathcal{C}]$ such that $\Theta \mathcal{D}^{*} \cong \mathbb{G}^{*}$.

4'. There exists an object $W$ of $\mathcal{D}$ such that $!^{W}: W \longrightarrow 1$ is an effective descent morphism and for every morphism $f: Y \longrightarrow W$ the morphism $\left(f, \eta_{Y}\right)$ : $Y \longrightarrow W \times_{\mathcal{D}^{*} \Sigma_{\mathcal{D}} W} \mathcal{D}^{*} \Sigma_{\mathcal{D}} Y$ is a regular monomorphism.

This result is first observed in [T14].

Proof. The functor $\left(\Sigma_{\mathcal{D}}\right)_{W}$ factors as $\Sigma_{\eta_{W}}: \mathcal{D} / W \longrightarrow \mathcal{D} / \mathcal{D}^{*} \Sigma_{\mathcal{D}} W$ followed by $(\Sigma \mathcal{D})_{\Sigma_{\mathcal{D}} W}$ (i.e. the left adjoint of $\Sigma_{\mathcal{D}} \dashv \mathcal{D}^{*}$ sliced at $\Sigma_{\mathcal{D}} W$ ). It has been observed already that $\Sigma_{\eta_{W}} \dashv \eta_{W}^{*}$ satisfies Frobenius reciprocity (as it is the pullback adjunction of a morphism in a cartesian category) and $\left(\Sigma_{\mathcal{D}}\right)_{\Sigma_{\mathcal{D}} W} \dashv\left(\mathcal{D}^{*}\right)_{\Sigma_{\mathcal{D}} W}$ satisfies Frobenius reciprocity by assumption. Frobenius reciprocity is closed under composition of adjunctions and so $\left(\Sigma_{\mathcal{D}}\right)_{W}: \mathcal{D} / W \longrightarrow \mathcal{C} / \Sigma_{\mathcal{D}} W$ is the left adjoint of an adjunction that satisfies Frobenius reciprocity. It can be seen that $\left(f, \eta_{Y}\right)$ is the unit of this adjunction at $Y_{f}$. Hence $\left(\Sigma_{\mathcal{D}}\right)_{W}$ is an equivalence by application of the Lemma 4.1 because $\left(\Sigma_{\mathcal{D}}\right)_{W}(1) \cong 1$ and $\left(f, \eta_{Y}\right)$ is a regular monomorphism for each $Y_{f}$. Conversely it is clear that if $\left(\Sigma_{\mathcal{D}}\right)_{W}$ is an equivalence then the unit is a regular monomorphism. 
In contrast to what is true if we only assume connected components:

Proposition 10.3. Let $\mathbb{G}$ and $\mathbb{H}$ be two internal groupoids in $\mathcal{C}$.

(i) If $\mathbb{H}$ is stably Frobenius then the functor $\pi_{2}^{*}:[\mathbb{G}, \mathcal{C}] \longrightarrow[\mathbb{H} \times \mathbb{G}, \mathcal{C}]$, corresponding to the projection functor $\pi_{2}: \mathbb{H} \times \mathbb{G} \longrightarrow \mathbb{G}$, has a left adjoint, $\Sigma_{\pi_{2}}$, such that the resulting adjunction is stably Frobenius.

(ii) If $\mathbb{F}: \mathbb{H} \longrightarrow \mathbb{G}$ is an internal functor and $\mathbb{H}$ is stably Frobenius then $\mathbb{F}^{*}:[\mathbb{G}, \mathcal{C}] \longrightarrow[\mathbb{H}, \mathcal{C}]$ has a left adjoint $\Sigma_{\mathbb{F}}$ such that $\Sigma_{\mathbb{F}} \dashv \mathbb{F}^{*}$ is stably Frobenius and over $\mathcal{C}$.

(iii) If $\mathbb{G}$ and $\mathbb{H}$ are both stably Frobenius then $\mathbb{G} \times \mathbb{H}$ is stably Frobenius.

Proof. (i) Given a $\mathbb{H} \times \mathbb{G}$-object $\left(P_{(p, q)}, c, d\right)$ notice that $\Sigma_{\mathbb{H}}\left(P_{p}, c\right)$ can be turned into a $\mathbb{G}$-object as it is the codomain of a pullback stable regular epimorphism by assumption that $\mathbb{H}$ is stably Frobenius (and it is over $G_{0}$ as $q$ is $c$-invariant). Define $\Sigma_{\pi_{2}}\left(P_{(p, q)}, c, d\right)$ to be this $\mathbb{G}$-object, extending to homomorphisms in the obvious manner. The remainder of the proof of (i) is then routine from construction.

(ii) Apply (i) and Lemma 9.1, noting that $\mathbb{F}^{*}$ factors as $\left(I d_{\mathbb{H}}, \mathbb{F}\right)^{*} \pi_{2}^{*}$. The claim of 'over $\mathcal{C}$ ' is clear from the definition of $\mathbb{F}^{*}$.

(iii) Verify that $(\mathbb{H} \times \mathbb{G})^{*} \cong \pi_{2}^{*} \mathbb{G}^{*}$ and then apply (i) and the assumption that $\mathbb{G}$ is stably Frobenius.

Internal essential equivalences between stably Frobenius groupoids give rise to equivalences at the level of categories of $\mathbb{G}$-objects:

Proposition 10.4. Given an internal functor $\mathbb{F}: \mathbb{H} \longrightarrow \mathbb{G}$ between two internal groupoids with $\mathbb{H}$ stably Frobenius, the following are equivalent:

(a) $\mathbb{F}$ is an essential equivalence.

(b) $\mathbb{F}$ is fully faithful and $d_{0}^{\mathbb{G}} \pi_{2}: H_{0} \times_{G_{0}} G_{1} \longrightarrow G_{0}$ is a regular epimorphism.

(c) $\mathbb{F}^{*}:[\mathbb{G}, \mathcal{C}] \longrightarrow[\mathbb{H}, \mathcal{C}]$ is an equivalence.

Proof. (a) implies (b) is trivial as effective descent morphisms are regular epimorphisms.

For (b) implies (c), by (ii) of the last Proposition there is a stably Frobenius adjunction $\Sigma_{\mathbb{F}} \dashv \mathbb{F}^{*}$ and we apply Lemma 4.1

The underlying object of $\Sigma_{\left(I d_{\mathbb{H}}, \mathbb{F}\right)}(1)$ is $H_{0} \times G_{0} G_{1} \stackrel{I d \times d_{0}^{\mathbb{G}}}{\longrightarrow} H_{0} \times G_{0}$ (i.e. $\left\{\left(x^{\mathbb{H}}, g_{0}\right) \mid d_{1}^{\mathbb{G}} g_{0}=\right.$ $\left.F_{0}\left(x^{\mathbb{H}}\right)\right\}$ with $\left.\left(x^{\mathbb{H}}, g_{0}\right) \mapsto\left(x^{\mathbb{H}}, d_{0}^{\mathbb{G}} g_{0}\right)\right)$ and the $\mathbb{H}$-action is $\left(h,\left(x, g_{0}\right)\right) \mapsto\left(d_{1}^{\mathbb{H}} h, F_{1} h g_{0}\right)$ (see the proof of Lemma 9.1, the action on the terminal object 1 of $[\mathbb{H}, \mathcal{C}]$ is $d_{1}^{\mathbb{H}}$ for any internal groupoid $\mathbb{H}$ ). The domain of the underlying object of $\Sigma_{\mathbb{F}} 1$ is the coequalizer of $d_{1}^{\mathbb{H}} \times m^{\mathbb{G}}\left(F_{1} \pi_{1}, \pi_{2}\right), d_{0}^{\mathbb{H}} \times I d_{G_{1}}: H_{1} \times G_{0} G_{1} \longrightarrow H_{0} \times_{G_{0}} G_{1}$. The unique $\mathbb{G}$-homomorphism $\Sigma_{\mathbb{F}} 1 \longrightarrow 1$ is given by the second factor of the factorization of $d_{0}^{\mathbb{G}} \pi_{2}: H_{0} \times_{G_{0}} G_{1} \longrightarrow G_{0}$ through this coequalizer. Any regular epimorphism is the coequalizer of its kernal pair, so we can show that if $d_{0}^{\mathbb{G}} \pi_{2}$ is a regular epimorphism then $\Sigma_{\mathbb{F}} 1 \cong 1$ by showing that the kernal pair of $d_{0}^{\mathbb{G}} \pi_{2}$ factors through (and is factored through by) the coequalizer that determines $\Sigma_{\mathbb{F}} 1$. Certainly $d_{1}^{\mathbb{H}} \times m^{\mathbb{G}}\left(F_{1} \pi_{1}, \pi_{2}\right), d_{0}^{\mathbb{H}} \times I d_{G_{1}}$ factors through $\pi_{1}, \pi_{2}$ : $\left(H_{0} \times G_{0} G_{1}\right) \times_{G_{0}}\left(H_{0} \times G_{0} G_{1}\right)$ as both maps are over $G_{0}$. But we also know that $\left(d_{0}^{\mathbb{H}}, d_{1}^{\mathbb{H}}, F_{1}\right): H_{1} \longrightarrow\left(H_{0} \times H_{0}\right) \times_{\left(G_{0} \times G_{0}\right)} G_{1}$ has an inverse, $\Phi$ say, by the assumption that $\mathbb{F}$ is fully faithful. Send $\left[\left(x_{1}^{\mathbb{H}}, g_{1}\right),\left(x_{2}^{\mathbb{H}}, g_{2}\right)\right]$, from the kernal pair of $d_{0}^{\mathbb{H}} \pi_{2}$, to $\left(\Phi\left(x_{1}^{\mathbb{H}}, x_{2}^{\mathbb{H}}, g_{2} g_{1}^{-1}\right), g_{1}\right)$ to verify that the kernal pair factors through $H_{1} \times G_{0} G_{1}$ as required. Therefore $\Sigma_{\mathbb{F}} 1 \cong 1$. 
For any $\mathbb{H}$-object $Y=\left(p: Y \longrightarrow H_{0}, b: H_{1} \times_{H_{0}} Y \longrightarrow Y\right)$ the underlying object over $G_{0}$ determined by the $\mathbb{G}$-object $\Sigma_{\mathbb{F}} Y$ is the codomain of the coequalizer $n: Y \times_{G_{0}} G_{1} \longrightarrow \Sigma_{\mathbb{H}}\left(Y \times_{G_{0}} G_{1}\right)$. That the unit $\eta_{Y}$ is

$$
Y \stackrel{\left(I d_{Y}, e^{\mathbb{G}} F_{0} p\right)}{\longrightarrow} Y \times_{G_{0}} G_{1} \stackrel{\left(p \pi_{1}, n\right)}{\longrightarrow} H_{0} \times_{G_{0}} \Sigma_{\mathbb{H}}\left(Y \times_{G_{0}} G_{1}\right)
$$

follows from our construction of $\Sigma_{\left(I d_{\mathbb{H}}, \mathbb{F}\right)} \dashv\left(I d_{\mathbb{H}}, \mathbb{F}\right)^{*}$ and of $\Sigma_{\pi_{2}} \dashv \pi_{2}^{*}$.

To complete (b) imples (c), we show that each unit $\eta_{Y}: Y \longrightarrow \mathbb{F}^{*} \Sigma_{\mathbb{F}} Y$ is a regular monomorphism if $\mathbb{F}$ is fully faithful. In fact it is simpler to prove the stronger result that $\eta_{Y}$ is an isomorphism. (The proof of this part has been taken from 5.13 of $\mathrm{M} 88 \mathrm{a}$.)

The coequalizer $q$ is pullback stable by assumption that $\mathbb{H}$ is stably Frobenius. In particular by pulling back along $F_{0}: H_{0} \longrightarrow G_{0}$ we see that (the underlying object over $H_{0}$ of) $\mathbb{F}^{*} \Sigma_{\mathbb{F}} Y$ is given by the coequalizer of

$$
H_{0} \times_{G_{0}}\left(H_{1} \times_{H_{0}} Y \times_{G_{0}} G_{1}\right) \Longrightarrow H_{0} \times_{G_{0}}\left(Y \times_{G_{0}} G_{1}\right)(*)
$$

where the top arrow is $(x, h, y, g) \mapsto(x, y, g)$ and the bottom arrow is $(x, h, y, g) \mapsto$ $\left(x, h y,\left(F_{1} h\right) g\right)$. Now $H_{1} \times_{H_{0}} Y=\left\{(h, y) \mid p y \stackrel{h}{\longrightarrow} d_{1}^{\mathbb{H}} h\right\}$ maps to $H_{0} \times{ }_{G_{0}}\left(Y \times_{G_{0}}\right.$ $\left.G_{1}\right)=\left\{(x, y, g) \mid F_{0} x \stackrel{g}{\longrightarrow} F_{0} p y\right\}$ via $(h, y) \mapsto\left(p h y, y,\left(F_{1} h\right)^{-1}\right)$, and this is an isomorphism as $\mathbb{F}$ is fully faithful. Also $H_{1} \times_{H_{0}} H_{1} \times_{H_{0}} Y=\left\{\left(h_{1}, h_{2}, y\right) \mid p y \stackrel{h_{2}}{\longrightarrow} d_{1}^{\mathbb{H}} h_{2} \stackrel{h_{1}}{\longrightarrow} d_{1}^{\mathbb{H}} h_{1}\right\}$ maps to $H_{0} \times_{G_{0}}\left(H_{1} \times_{H_{0}} Y \times_{G_{0}} G_{1}\right)=\left\{(x, h, y, g) \mid F_{0} x \stackrel{g}{\longrightarrow} F_{0} p y, p y \stackrel{h}{\longrightarrow} d_{1}^{\mathbb{H}} h\right\}$ via $\left(h_{1}, h_{2}, y\right) \mapsto\left(p h_{1} h_{2} y, h_{2}^{-1}, h_{2} y,\left(F_{1} h_{1}\right)^{-1}\right)$ and this too is an isomorphism as $\mathbb{F}$ is fully faithful. But using these two isomorphisms it can be checked that the pair $(*)$ is isomorphic to the pair

$$
H_{1} \times_{H_{0}}\left(H_{1} \times_{H_{0}} Y\right) \underset{m^{\mathbb{H}} \times I d_{Y}}{\stackrel{I d_{H_{1}} \times b}{\longrightarrow}} H_{1} \times_{H_{0}} Y(* *)
$$

and so their coequalizers are isomorphic. Since the coequalizer of the second pair $(* *)$ is $b: H_{1} \times_{H_{0}} Y \longrightarrow Y$ (it is split by $\left(e^{\mathbb{H}} p, I d_{Y}\right)$ ) we have that there is an isomorphism from $Y$ to $\mathbb{F}^{*} \Sigma_{\mathbb{F}} Y$; it is clear from construction that this isomorphism is $\eta_{Y}$.

For (c) implies (a), by considering the unit $\eta_{Y}$ at $Y=\mathbb{T}_{\mathbb{H}} 1$ we see that if the unit is an isomorphism then $\mathbb{F}$ is fully faithful. Equivalences preserve effective descent morphisms and so ! : $\Sigma_{\mathbb{F}} \mathbb{T}_{\mathbb{H}} 1 \longrightarrow 1$ is an effective descent morphism by Lemma 7.4. Then, by part 2. of Lemma 4.2 applied to $\mathbb{T}_{\mathbb{G}} \dashv U_{\mathbb{G}}$, we see that $U_{\mathbb{G}} \Sigma_{\mathbb{F}} T_{\mathbb{H}} 1 \stackrel{U_{\mathbb{G}} !}{\longrightarrow} 1$ is an effective descent morphism. This completes the proof as this last map is $d_{0}^{\mathbb{G}} \pi_{2}: H_{0} \times_{G_{0}} G_{1} \longrightarrow G_{0}\left(\right.$ recall: $\Sigma_{H_{0}} U_{\mathbb{H}} \cong \Sigma_{\mathbb{H}} \mathbb{T}_{\mathbb{H}} U_{\mathbb{H}} \cong \Sigma_{\mathbb{H}}\left[\mathbb{T}_{\mathbb{H}} 1 \times(-)\right]$ - quotienting out $H_{1}$ returns $H_{0}$ and the quotient is pullback stable).

The weakening of the definition of essential equivalence in this last proposition (part (b)), which is available as we are restricting to stably Frobenius groupoids, works similarly for Hilsum-Skandalis maps. We show this in the following result, but note that it is not used elsewhere in the paper.

Proposition 10.5. Let $\mathbb{H}$ and $\mathbb{G}$ be two groupoids internal to a category $\mathcal{C}$ with $\mathbb{G}$ stably Frobenius. If $\left(P \stackrel{(p, q)}{\longrightarrow} H_{0} \times G_{0}, c: H_{1} \times_{H_{0}} P \longrightarrow P, d: G_{1} \times_{G_{0}} P \longrightarrow P\right)$ is a $\mathbb{H} \times \mathbb{G}$-object then it is a Hilsum-Skandalis map if and only if

(i) $\left(d, \pi_{2}\right): G_{1} \times_{G_{0}} P \longrightarrow P \times_{H_{0}} P$ is an isomorphism, and

(ii) $p: P \longrightarrow H_{0}$ is a regular epimorphism. 
Proof. As an effective descent morphism is a regular epirmorphism, to complete this proof we must just show that $p$ is an effective descent morphism if conditions (i) and (ii) hold. Firstly, if $p$ is a regular epimorphism then it is the coequalizer of its kernel pair. But this kernel pair is isomorphic to $d, \pi_{2}: G_{1} \times_{G_{0}} P \Longrightarrow P$ and their coequalizer is $G_{1} \times{ }_{G_{0}} P \stackrel{n}{\longrightarrow} \Sigma_{\mathbb{G}}\left(P_{q}, c\right)$ which, by assumption that $\mathbb{G}$ is stably Frobenius, is pullback stable. Therefore $p$ is a pullback stable regular epimorphism. Note that $H_{0} \cong \Sigma_{\mathbb{G}}\left(P_{q}, d\right)$ and under this isomorphism the unit of $\Sigma_{\mathbb{G}} \dashv \mathbb{G}^{*}$ at $\left(P_{q}, d\right)$ is $P \stackrel{(q, p)}{\longrightarrow} G_{0} \times H_{0}$.

It is well known that $p^{*}$ reflects isomorphisms for any pullback stable regular epimorphism $p$. (For completeness we recall the proof: if $\theta: A_{a} \longrightarrow B_{b}$, a morphism of $\mathcal{C} / H_{0}$, is such that $p^{*} \theta$ is an isomorphism, then $\pi_{2}: P \times_{H_{0}} A \longrightarrow A$ and $\pi_{2}^{\prime}: P \times_{H_{0}} B \longrightarrow B$ are both regular epimorphisms by the assumption that $p$ is a pullback stable regular epimorphism. In particular $\pi_{2}$ and $\pi_{2}^{\prime}$ are coequalizers of their respective kernel pairs. But their kernel pairs can be seen to be isomorphic as $p^{*} \theta$ is an isomorphism and so $A \cong B$. Proving additionally that this isomorphism is $\theta$ is clear from construction.)

As $P_{(p, q)}$ is (the underlying object of) a $\mathbb{H} \times \mathbb{G}$-object $(p, q):\left(P_{p}, e\right) \longrightarrow \mathbb{G}^{*} H_{0}$ is a $\mathbb{G}$-homomorphism. Now because $\Sigma_{\mathbb{G}}\left(P_{q}, e\right)$ is given by the coequalizer of $e, \pi_{2}$ : $G_{1} \times_{G_{0}} P \Longrightarrow P$ and condition (i) holds by assumption, $\Sigma_{\mathbb{G}}\left(P_{q}, e\right)$ is given by the coequalizer of the kernel pair $p$, which is $p$ itself as $p$ is a regular epimorpishm. From this it is clear that the adjoint transpose of $(p, q)$ is (isomorphic to) the identity on $H_{0}$.

Now, say $f, g: A_{a} \Longrightarrow B_{b}$ are a pair of morphisms of $\mathcal{C} / H_{0}$ that are $p^{*}$-split with $r: P \times_{H_{0}} B \longrightarrow Q$ the coequalizer of $I d_{P} \times f$ and $I d_{P} \times g$. Certainly $q \pi_{1}: P \times_{H_{0}} B \longrightarrow G_{0}$ composes equally with $I d_{P} \times f$ and $I d_{P} \times g$ and so factors via $r$; say as $t r$, where $t: Q \longrightarrow G_{0}$. The objects $\left(P \times_{H_{0}} A\right)_{q \pi_{1}}$ and $\left(P \times_{H_{0}} B\right)_{q \pi_{1}}$ of $\mathcal{C}$ are the underlying objects of the $\mathbb{G}$-objects $\left(P_{q}, d\right) \times_{\mathbb{G}^{*} H_{0}} \mathbb{G}^{*} A$ and $\left(P_{q}, d\right) \times_{\mathbb{G}^{*} H_{0}} \mathbb{G}^{*} B$ (where the pullback in $[\mathbb{G}, \mathcal{C}]$ is along $\left.P \stackrel{(p, q)}{\longrightarrow} H_{0} \times G_{0}\right)$. But $r$ is part of a split coequalizer diagram, preserved by any functor, in particular $G_{1} \times G_{0}(-)$, and so $\left(Q_{t}, k\right)$ is a $\mathbb{G}$-object with an action $k$, since $I d_{P} \times f$ and $I d_{P} \times g$ are both $\mathbb{G}$-homomorphisms; $r$ is their coequalizer in $[\mathbb{G}, \mathcal{C}]$. Apply $\Sigma_{\mathbb{G}}$, a left adjoint, to this coequalizer, use Frobenius reciprocity of $\Sigma_{\mathbb{G}} \dashv \mathbb{G}^{*}$ at $H_{0}$ and recall our earlier observation that the adjoint transpose of $(p, q):\left(P_{p}, e\right) \longrightarrow \mathbb{G}^{*} H_{0}$ is the identity on $H_{0}$ as $(p, q)$ is isomorphic to the unit of the adjunction. This shows that $\Sigma_{\mathbb{G}}\left(Q_{t}, k\right)$ is a coequalizer of $f, g$. It can be checked that this coequalizer is over $H_{0}$ as $b \pi_{2}: P \times_{H_{0}} B \longrightarrow H_{0}$ is $\mathbb{G}$-invariant (and $I d_{G_{1}} \times r$ is an epimorphism) so the unique factorization of $b \pi_{2}$ through $r$ also factors through $n: Q \longrightarrow \Sigma \mathbb{G}\left(Q_{t}, k\right)$. It also follows that $Q \cong P \times_{H_{0}} \Sigma_{\mathbb{G}}\left(Q_{t}, k\right)$ as the coequalizers determined by $\Sigma_{\mathbb{G}}$ are pullback stable in the strong sense (recall, by considering kernel pairs, that for any map $s$, if $s$ is the coequalizer in a coqualizer diagram and $s$ is a pullback stable regular epimorphism then the coequalizer diagram itself is pullback stable). This completes a verification of the conditions of Beck's theorem for $p^{*}$ and so $p$ is of effective descent.

\section{Hilsum-Skandalis maps as Stably Frobenius adjunctions}

We can now prove our first main result which is that between stably Frobenius groupoids, all Hilsum-Skandalis maps arise from stably Frobenius adjunctions. 
Theorem 11.1. If $\mathfrak{P}: \mathbb{H} \longrightarrow \mathbb{G}$ is a Hilsum-Skandalis map, with $\mathbb{H}$ and $\mathbb{G}$ stably Frobenius, then there exists a stably Frobenius adjunction $L^{\mathfrak{P}} \dashv R^{\mathfrak{P}}:[\mathbb{H}, \mathcal{C}] \rightleftarrows[\mathbb{G}, \mathcal{C}]$ over $\mathcal{C}$ such that $\mathfrak{P} \cong \mathfrak{P}_{L^{\mathfrak{P}}, R^{\mathfrak{P}}}$.

Proof. By Lemma 9.4 the projection functor $\mathfrak{p}: \mathbb{P} \longrightarrow \mathbb{H}$ is an essential equivalence, where $\mathbb{P}$ is the internal groupoid corresponding to $\mathfrak{P}$ (use Lemma 7.5 $[\mathbb{P}, \mathcal{C}] \stackrel{\simeq}{\longrightarrow}[\mathbb{H} \times \mathbb{G}, \mathcal{C}] / \mathfrak{P}$ over $\mathcal{C}$ ). Now $\Sigma_{\mathfrak{P}} \dashv \mathfrak{P}^{*}$ is stably Frobenius (it is a pullback adjunction) so we can conclude that $\mathbb{P}$ is stably Frobenius, because $\mathbb{H} \times \mathbb{G}$ is stably Frobenius (by (iii) of Proposition 10.3). As $\mathbb{P}$ is stably Frobenius we can apply Proposition 10.4 to conclude that $[\mathbb{H}, \mathcal{C}] \stackrel{\mathfrak{p}^{*}}{\longrightarrow}[\mathbb{P}, \mathcal{C}]$ is an equivalence. We therefore have an equivalence $\phi:[\mathbb{H}, \mathcal{C}] \stackrel{\simeq}{\longrightarrow}[\mathbb{H} \times \mathbb{G}, \mathcal{C}] / \mathfrak{P}$. Given an $\mathbb{H}$-object $\left(Y_{g}, b\right)$, its image is the projection $\pi_{1}: P \times_{H_{0}} Y \longrightarrow P$ (where the action on $P \times_{H_{0}} Y$ is determined by $(g, h)(x, y)=(g h x, h y))$.

Define $L^{\mathfrak{P}} \dashv R^{\mathfrak{P}}$ as

$$
[\mathbb{H}, \mathcal{C}] \simeq[\mathbb{H} \times \mathbb{G}, \mathcal{C}] / \mathfrak{P} \underset{\mathfrak{P}^{*}}{\stackrel{\Sigma_{\mathfrak{P}}}{\rightleftarrows}}[\mathbb{H} \times \mathbb{G}, \mathcal{C}] \underset{\pi_{2}^{*}}{\stackrel{\Sigma_{\pi_{2}}}{\rightleftarrows}}[\mathbb{G}, \mathcal{C}]
$$

where the final adjunction is from (i) of Proposition 10.3. This adjunction is stably Frobenius and over $\mathcal{C}$.

Say $\left(P \stackrel{(p, q)}{\longrightarrow} H_{0} \times G_{0}, c: H_{1} \times_{H_{0}} P \longrightarrow P, d: G_{1} \times_{G_{0}} P \longrightarrow P\right)$ is the underlying data of $\mathfrak{P}$. The image of the $\mathbb{H}$-object $\left(P_{p}, c\right)$ under the equivalence in the display (*) is the morphism $P \times_{H_{0}} P \stackrel{\pi_{1}}{\longrightarrow} P$. The image of $\mathbb{T}_{\mathbb{G}} 1$ under $\mathfrak{P}^{*} \pi_{2}^{*}$ is the pullback of $d_{1}^{\mathbb{G}} \times I d_{H_{0}}: G_{1} \times H_{0} \longrightarrow G_{0} \times H_{0}$ along $P \stackrel{(q, p)}{\longrightarrow} G_{0} \times H_{0}$ which is $\pi_{2}: G_{1} \times_{G_{0}} P \longrightarrow P$. But $G_{1} \times_{G_{0}} P \longrightarrow P \times_{H_{0}} P$ given by $\left(g_{0}, x\right) \mapsto\left(x, g_{0}^{-1} x\right)$ is an $\mathbb{H} \times \mathbb{G}$-isomorphism as $\mathfrak{P}$ is a Hilsum-Skandalis map and so $\left(P_{p}, c\right) \cong R^{\mathfrak{P}} \mathbb{T}_{\mathbb{G}} 1$.

Further, the image of $\mathbb{T}_{\mathbb{H}} 1$ under the equivalence in the display $\left(^{*}\right)$ is the $\mathbb{H} \times \mathbb{G}$ homomorphism $\pi_{1}: P \times_{H_{0}} H_{1} \longrightarrow P$. But $\Sigma_{\pi_{2}}$ quotients out the $\mathbb{H}$-action and when applied to (the $\mathbb{H} \times \mathbb{G}$-object whose underlying object is) $P \times_{H_{0}} H_{1}$ returns $\left(P_{q}, d\right)$ as the quotienting map $P \times_{H_{0}} H_{1} \longrightarrow P$ can be seen to be $\left(x, h_{0}\right) \mapsto h_{0}^{-1} x$ which can be split using the identity of $\mathbb{H}$. Hence $\left(P_{q}, d\right) \cong L^{\mathfrak{P}} \mathbb{T}_{\mathbb{H}} 1$ and so $\mathfrak{P} \cong$ $\mathfrak{P}_{L^{\mathfrak{P}}, R^{\mathfrak{P}}}$.

The reader may find it odd that we have not mentioned profunctors. Whilst parts of the proof could usefully reference constructions from the well established theory of profunctors, notably the construction of $L^{\mathfrak{P}} \dashv R^{\mathfrak{P}}$ from $\mathfrak{P}$, I was not able to easily adapt the theory of profunctors to get the result.

Corollary 11.2. If $\mathbb{H}$ and $\mathbb{G}$ are two stably Frobenius groupids, then there exists an equivalence $\theta:[\mathbb{H}, \mathcal{C}] \longrightarrow[\mathbb{G}, \mathcal{C}]$ over $\mathcal{C}$ if and only if there is a span $\mathbb{H} \stackrel{\mathfrak{p}}{\longleftarrow} \mathbb{P} \stackrel{\mathfrak{q}}{\longrightarrow} \mathbb{G}$ such that both $\mathfrak{p}$ and $\mathfrak{q}$ are essential equivalences.

Proof. If we have such a span then by apply Proposition 10.4 twice to construct the required equivalence. In the other direction if $\theta$ is an equivalence then $\theta \dashv \theta^{-1}$ and $\theta^{-1} \dashv \theta$. Apply the Theorem twice and notice that we get the same span both times.

Two internal groupoids, internal to $\mathcal{C}$, are said to be Morita equivalent if their categories of $\mathbb{G}$-objects are equivalent over $\mathcal{C}$. The corollary characterizes Morita equivalence for stably Frobenius groupoids. 
We now extend the Theorem to morphisms and so make good our claim that Hilsum-Skandalis maps between stably Frobenius groupoids can be represented by stably Frobenius adjunctions.

Theorem 11.3. If $\mathbb{H}$ and $\mathbb{G}$ are two internal stably Frobenius groupoids, then the category of Hilsum-Skandalis maps from $\mathbb{H}$ to $\mathbb{G}$ is equivalent to the category of stably Frobenius adjunctions $L \dashv R:[\mathbb{H}, \mathcal{C}] \rightleftarrows[\mathbb{G}, \mathcal{C}]$ over $\mathcal{C}$.

Proof. We first verify that the assignment of Hilsum-Skandalis maps from adjunctions over $\mathcal{C}$ (i.e. $\left.(L \dashv R) \mapsto \mathfrak{P}_{L, R}\right)$ is functorial. Say we have two adjunctions, $L_{i} \dashv$ $R_{i}, i=1,2$ over $\mathcal{C}$. Then any natural transformation between the left adjoints (say $\alpha$ from $L_{1}$ to $L_{2}$ ) gives rise to a natural transformation between the right adjoints; say $\beta: R_{2} \longrightarrow R_{1}$. Explicitly, $\beta$ is $R_{2} \stackrel{\eta^{1}}{\longrightarrow} R_{1} L_{1} R_{2} \stackrel{R_{1} \alpha_{R_{2}}}{\longrightarrow} R_{1} L_{2} R_{2} \stackrel{R_{1} \epsilon^{2}}{\longrightarrow} R_{1}$ where $\eta^{i}$ and $\epsilon^{i}$ are the unit and counit respectively of $L_{i} \dashv R_{i}$ for $i=1,2$; from this definition we always have $\epsilon^{1} L_{1} \beta=\epsilon^{2} \alpha_{R_{2}}$. Then we note that we have $\alpha_{\mathbb{T}_{\mathbb{H}} 1}: L_{1} \mathbb{T}_{\mathbb{H}} 1 \longrightarrow L_{2} \mathbb{T}_{\mathbb{H}} 1$ which is a $\mathbb{G}$-homomorphism. In fact it must be an isomorphism by application of part (a) of Proposition 8.2 , We also have $\beta_{\mathbb{T}_{\mathbb{G}} 1}$ : $R_{2} \mathbb{T}_{\mathbb{G}} 1 \longrightarrow R_{1} \mathbb{T}_{\mathbb{G}} 1$ which is an $\mathbb{H}$-homomorphism. This looks like it is going in the wrong direction, but if we can show that the canonical isomorphism $\psi_{2}: \Sigma_{H_{0}} U_{\mathbb{H}} R_{2} \mathbb{T}_{\mathbb{G}} 1 \longrightarrow \Sigma_{G_{0}} U_{\mathbb{G}} L_{2} \mathbb{T}_{\mathbb{H}} 1$ factors as

$\Sigma_{H_{0}} U_{\mathbb{H}} R_{2} \mathbb{T}_{\mathbb{G}} 1 \stackrel{\Sigma_{H_{0}} U_{\mathbb{H}} \beta_{\mathbb{T}_{\mathbb{G}}} 1}{\longrightarrow} \Sigma_{H_{0}} U_{\mathbb{H}} R_{1} \mathbb{T}_{\mathbb{G}} 1 \stackrel{\psi_{1}}{\longrightarrow} \Sigma_{G_{0}} U_{\mathbb{G}} L_{1} \mathbb{T}_{\mathbb{H}} 1 \stackrel{\Sigma_{G_{0}} U_{\mathbb{G}} \alpha_{\mathbb{T}_{\mathbb{H}}}}{\longrightarrow} \Sigma_{G_{0}} U_{\mathbb{G}} L_{2} \mathbb{T}_{\mathbb{H}} 1$

then we will be done as then $\beta_{\mathbb{T}_{\mathbb{G}} 1}^{-1}$ can be defined, is a $\mathbb{H}$-homomorphism and corresponds to $\alpha_{\mathbb{T}_{\mathbb{H}} 1}$ under the canonical isomorphisms. Now, up to canonical isomorphisms (determined by $\mathbb{G}$ and $\mathbb{H}$ and not by the adjunctions being over $\mathcal{C}$ ) $\psi_{i}$, for $i=1,2$, is

$\Sigma_{\mathbb{H}}\left(\mathbb{T}_{\mathbb{H}} 1 \times R_{i} \mathbb{T}_{\mathbb{G}} 1\right) \stackrel{\tau_{i}^{-1}}{\longrightarrow} \Sigma_{\mathbb{G}} L_{i}\left(\mathbb{T}_{\mathbb{H}} 1 \times R_{i} \mathbb{T}_{\mathbb{G}} 1\right) \stackrel{\Sigma_{\mathbb{G}}\left(\epsilon_{\mathbb{T}_{\mathbb{G}}}^{i} L_{i} \pi_{2}, L_{i} \pi_{1}\right)}{\longrightarrow} \Sigma_{\mathbb{G}}\left(\mathbb{T}_{\mathbb{G}} 1 \times L_{i} \mathbb{T}_{\mathbb{H}} 1\right)$

where $\tau_{i}$ are the natural isomorphisms that exist as the adjunctions are over $\mathcal{C}$. Under the canonical isomorphism determined by $\mathbb{G}, \alpha_{\mathbb{T}_{\mathbb{H}} 1}$ corresponds to $\Sigma_{\mathbb{G}}\left(I d_{\mathbb{T}_{\mathbb{G}} 1} \times\right.$ $\left.\alpha_{\mathbb{T}_{\mathbb{H}} 1}\right)$ and similarly $\beta_{\mathbb{T}_{\mathbb{G}} 1}$ corresponds to $\Sigma_{\mathbb{H}}\left(I d_{\mathbb{T}_{\mathbb{H}} 1} \times \beta_{\mathbb{T}_{\mathbb{G}} 1}\right)$. To prove the factorisation we check that $\Sigma_{\mathbb{G}}\left(\epsilon_{\mathbb{T}_{\mathbb{G}}}^{2} L_{2} \pi_{2}, L_{2} \pi_{1}\right) \tau_{2}^{-1}$

$$
\begin{aligned}
& =\Sigma_{\mathbb{G}}\left(\epsilon_{\mathbb{T}_{\mathbb{G}} 1}^{2} L_{2} \pi_{2}, L_{2} \pi_{1}\right) \Sigma_{\mathbb{G}} \alpha_{\mathbb{T}_{\mathbb{H}} 1 \times R_{2} \mathbb{T}_{\mathbb{G} 1} \tau_{1}}(\text { as } \alpha \text { is over } \mathcal{C}) \\
& =\Sigma_{\mathbb{G}}\left(\epsilon_{\mathbb{T}_{\mathbb{G}} 1}^{2} \times I d_{L_{2} \mathbb{T}_{\mathbb{H}} 1}\right) \Sigma_{\mathbb{G}}\left(\alpha_{R_{2} \mathbb{T}_{\mathbb{G}} 1} \times \alpha_{\mathbb{T}_{\mathbb{H}} 1}\right) \Sigma_{\mathbb{G}}\left(L_{1} \pi_{2}, L_{1} \pi_{1}\right) \tau_{1}^{-1} \\
& =\Sigma_{\mathbb{G}}\left(I d_{\mathbb{T}_{\mathbb{G}} 1} \times \alpha_{\mathbb{T}_{\mathbb{H}} 1}\right) \Sigma_{\mathbb{G}}\left(\epsilon_{\mathbb{T}_{\mathbb{G}} 1}^{2} \times I d_{L_{1} \mathbb{T}_{\mathbb{H}} 1}\right) \Sigma_{\mathbb{G}}\left(\alpha_{R_{2} \mathbb{T}_{\mathbb{G}} 1} \times I d_{L_{1} \mathbb{T}_{\mathbb{H}} 1}\right) \Sigma_{\mathbb{G}}\left(L_{1} \pi_{2}, L_{1} \pi_{1}\right) \tau_{1}^{-1} \\
& =\Sigma_{\mathbb{G}}\left(I d_{\mathbb{T}_{\mathbb{G}} 1} \times \alpha_{\mathbb{T}_{\mathbb{H}} 1}\right) \Sigma_{\mathbb{G}}\left(\epsilon_{\mathbb{T}_{\mathbb{G}} 1}^{1} \times I d_{L_{1} \mathbb{T}_{\mathbb{H}} 1}\right) \Sigma_{\mathbb{G}}\left(L_{1} \beta_{\mathbb{T}_{\mathbb{G}} 1} \times I d_{L_{1} \mathbb{T}_{\mathbb{H}} 1}\right) \Sigma_{\mathbb{G}}\left(L_{1} \pi_{2}, L_{1} \pi_{1}\right) \tau_{1}^{-1} \\
& =\Sigma_{\mathbb{G}}\left(I d_{\mathbb{T}_{\mathbb{G}} 1} \times \alpha_{\mathbb{T}_{\mathbb{H}} 1}\right) \Sigma_{\mathbb{G}}\left(\epsilon_{\mathbb{T}_{\mathbb{G}} 1}^{1} \times I d_{L_{1} \mathbb{T}_{\mathbb{H}} 1}\right) \Sigma_{\mathbb{G}}\left(L_{1} \pi_{2}, L_{1} \pi_{1}\right) \Sigma_{\mathbb{G}} L_{1}\left(I d_{\mathbb{T}_{\mathbb{H}} 1} \times \beta_{\mathbb{T}_{\mathbb{G}} 1}\right) \tau_{1}^{-1} \\
& =\Sigma_{\mathbb{G}}\left(I d_{\mathbb{T}_{\mathbb{G}} 1} \times \alpha_{\mathbb{T}_{\mathbb{H}} 1}\right) \Sigma_{\mathbb{G}}\left(\epsilon_{\mathbb{T}_{\mathbb{G}} 1}^{1} L_{1} \pi_{2}, L_{1} \pi_{1}\right) \Sigma_{\mathbb{G}} L_{1}\left(I d_{\mathbb{T}_{\mathbb{H}} 1} \times \beta_{\mathbb{T}_{\mathbb{G}} 1}\right) \tau_{1}^{-1} \\
& =\Sigma_{\mathbb{G}}\left(I d_{\mathbb{T}_{\mathbb{G}} 1} \times \alpha_{\mathbb{T}_{\mathbb{H}} 1}\right) \Sigma_{\mathbb{G}}\left(\epsilon_{\mathbb{T}_{\mathbb{G}} 1}^{1} L_{1} \pi_{2}, L_{1} \pi_{1}\right) \tau_{1}^{-1} \Sigma_{\mathbb{H}}\left(I d_{\mathbb{T}_{\mathbb{H}} 1} \times \beta_{\mathbb{T}_{\mathbb{G}} 1}\right)
\end{aligned}
$$

where the second line is by naturality of $\alpha$ at $\pi_{1}: \mathbb{T}_{\mathbb{H}} 1 \times R_{2} \mathbb{T}_{\mathbb{G}} 1 \longrightarrow \mathbb{T}_{\mathbb{H}} 1$ and $\pi_{2}: \mathbb{T}_{\mathbb{H}} 1 \times R_{2} \mathbb{T}_{\mathbb{G}} 1 \longrightarrow R_{2} \mathbb{T}_{\mathbb{G}} 1$, the fourth line is from the definition of $\beta$ and the fifth line is naturality of $\beta$. The last line is naturality of $\tau_{1}$; we have been suppressing the objects in the notation for the $\tau$ s. So we can conclude that $(L \dashv R) \mapsto \mathfrak{P}_{L, R}$ is functorial. 
In the other direction to make $\mathfrak{P} \mapsto\left(L_{\mathfrak{P}} \dashv R_{\mathfrak{P}}\right)$ functorial say we have an $\mathbb{H} \times \mathbb{G}$ homomorphism $a: \mathfrak{P}_{1} \longrightarrow \mathfrak{P}_{2}$; in other words we have a morphism $a: P_{1} \longrightarrow P_{2}$ that is both an $\mathbb{H}$-homomorphism and a $\mathbb{G}$-homomorphism. Writing $L_{i} \dashv R_{i}$ for $L_{\mathfrak{P}_{i}} \dashv R_{\mathfrak{P}_{i}}$, by the last theorem $P_{i} \cong L_{i} \mathbb{T}_{\mathbb{H}} 1$ and so by Lemma 4.3 applied to $L_{i} \mathbb{T}_{\mathbb{H}} \dashv$ $U_{\mathbb{H}} R_{i}$ there is a natural transformation $\hat{a}: L_{1} \mathbb{T}_{\mathbb{H}} \longrightarrow L_{2} \mathbb{T}_{\mathbb{H}}$ over $\mathcal{C}$ that is uniquely determined by $\hat{a}_{1}=a^{\prime}$ where $a^{\prime} \cong a$ via $P_{i} \cong L_{i} \mathbb{T}_{\mathbb{H}} 1$. Explicitly, for every object $Y_{g}$ of $\mathcal{C} / H_{0}, \hat{a}_{Y_{g}}$ is defined using $a^{\prime} \times I d_{Y}: L_{1} \mathbb{T}_{\mathbb{H}} 1 \times_{\mathbb{H}^{*} H_{0}} \mathbb{H}^{*} Y \longrightarrow L_{2} \mathbb{T}_{\mathbb{H}} 1 \times_{\mathbb{H}^{*} H_{0}}$ $\mathbb{H}^{*} Y$ and $L_{i} \mathbb{T}_{\mathbb{H}} Y_{g} \cong L_{i} \mathbb{T}_{\mathbb{H}} 1 \times_{\mathbb{H}} H_{0} H^{*} Y$. But every $\mathbb{H}$-object $\left(Y_{g}, b\right)$ is a canonical coequalizer of a pair of arrows

$$
\mathbb{T}_{\mathbb{H}} U_{\mathbb{H}} \mathbb{T}_{\mathbb{H}} U_{\mathbb{H}}\left(Y_{g}, b\right) \underset{\epsilon_{\mathbb{H}} U_{\mathbb{H}}\left(Y_{g}, b\right)}{\stackrel{\mathbb{T}_{\mathbb{H}} b}{\longrightarrow}} \mathbb{T}_{\mathbb{H}} U_{\mathbb{H}}\left(Y_{g}, b\right) .
$$

where $\epsilon$ is the counit of $\mathbb{T}_{\mathbb{H}} \dashv U_{\mathbb{H}}$. Therefore as $L_{i}$ preserves coequalizers, $\hat{a}$ can be seen to define a natural transformation $\bar{a}: L_{1} \longrightarrow L_{2}$ over $\mathcal{C}$, uniquely determined by $\bar{a}_{\left(Y_{g}, b\right)} b=b \hat{a}_{Y_{g}}$ for all $\mathbb{H}$-objects $\left(Y_{g}, b\right)$, provided we can check that (a) $\hat{a}_{Y_{g}}\left(L_{1} \mathbb{T}_{\mathbb{H}} b\right)=\left(L_{2} \mathbb{T}_{\mathbb{H}} b\right) \hat{a}_{U_{\mathbb{H}} \mathbb{T}_{\mathbb{H}} Y_{g}}$ and (b) $\hat{a}_{Y_{g}}\left(L_{1} \epsilon_{\mathbb{T}_{\mathbb{H}} U_{H}\left(Y_{g}, b\right)}\right)=\left(L_{2} \epsilon_{\mathbb{T}_{H}} U_{\mathbb{H}}\left(Y_{g}, b\right)\right) \hat{a}_{U_{\mathbb{H}} \mathbb{T}_{\mathbb{H}} Y_{g}}$. That (a) is true is clear from naturality of $\hat{a}$. As for (b), recall the diagrams after Lemma 7.2 which show how $\epsilon_{\mathbb{T}_{\mathbb{H}} U_{\mathbb{H}}\left(Y_{g}, b\right)}$ can be identified with $\pi_{2} \times I d$ : $\mathbb{T}_{\mathbb{H}} 1 \times \mathbb{T}_{\mathbb{H}} 1 \times\left(Y_{g}, b\right) \longrightarrow \mathbb{T}_{\mathbb{H}} 1 \times\left(Y_{g}, b\right)$. Hence $L \epsilon_{\mathbb{T}_{\mathbb{H}} U_{\mathbb{H}}\left(Y_{g}, b\right)}$ can be identified with

$\Sigma_{\mathbb{G}}\left(\mathbb{T}_{\mathbb{G}} 1 \times L\left[\mathbb{T}_{\mathbb{H}} 1 \times \mathbb{T}_{\mathbb{H}} 1 \times\left(Y_{g}, b\right)\right]\right) \stackrel{\Sigma_{\mathbb{G}}\left(I d \times L\left[\pi_{2} \times I d\right]\right)}{\longrightarrow} \Sigma_{\mathbb{G}}\left(\mathbb{T}_{\mathbb{G}} 1 \times L\left[\mathbb{T}_{\mathbb{H}} 1 \times\left(Y_{g}, b\right)\right]\right)$

which (see the proof of Proposition 8.3) is isomorphic to:

$\Sigma_{\mathbb{H}}\left(\left[\mathbb{T}_{\mathbb{H}} 1 \times \mathbb{T}_{\mathbb{H}} 1 \times\left(Y_{g}, b\right)\right] \times R \mathbb{T}_{\mathbb{G}} 1\right) \stackrel{\Sigma_{\mathbb{H}}\left(\left[\pi_{2} \times I d\right] \times I d\right)}{\longrightarrow} \Sigma_{\mathbb{H}}\left(\left[\mathbb{T}_{\mathbb{H}} 1 \times\left(Y_{g}, b\right)\right] \times R \mathbb{T}_{\mathbb{G}} 1\right)$

But this last is the $\mathbb{H}$-action on $\left(Y_{g}, b\right) \times R \mathbb{T}_{\mathbb{G}} 1$ and so (b) holds because $a$ is also an $\mathbb{H}$-homomorphism (recalling how $\hat{a}$ is defined in terms of $a \times I d$ ). Hence $\mathfrak{P} \mapsto\left(L_{\mathfrak{P}} \dashv R_{\mathfrak{P}}\right)$ is functorial.

Now by applying (b) to $\left(Y_{g}, b\right)=1$ we see that $a^{\prime} L_{1} \epsilon_{\mathbb{T}_{\mathbb{H}} 1}=L_{2} \epsilon_{\mathbb{T}_{\mathbb{H}} 1} \hat{a}_{U_{\mathbb{H}} \mathbb{T}_{\mathbb{H}} 1}$ and so $a^{\prime}=\bar{a}_{\mathbb{T}_{\mathbb{H}} 1}$, from which it is clear that $(L \dashv R) \mapsto \mathfrak{P}_{L, R}$ is full. To show faithfulness notice that for $\alpha: L_{1} \longrightarrow L_{2}$ over $\mathcal{C}$, the natural transformation $\alpha_{\mathbb{T}_{\mathbb{H}}}: L_{1} \mathbb{T}_{\mathbb{H}} \longrightarrow L_{2} \mathbb{T}_{\mathbb{H}}$ is equal to $\widehat{\alpha_{\mathbb{T}_{\mathbb{H}}}}$ because they both agree at 1 (Lemma 4.3). By naturality of $\alpha$ at $b$ we then have $\alpha_{\left(Y_{g}, b\right)} L_{1} \mathbb{T}_{\mathbb{H}} b=b\left(\widehat{\alpha_{\mathbb{T}_{H}}}\right)_{\left(Y_{g}, b\right)}$ and so $\alpha$ is uniquely determined by $\alpha_{\mathbb{T}_{\mathbb{H}}}$.

The next Proposition is an application of the characterisation of morphisms between Hilsum-Skandlis maps as natural transformations (neccesarily isomorphisms) between stably Frobenius adjunctions. Recall that if $\mathbb{G}$ is an internal groupoid, in $\mathcal{C}$, and $I$ is an object of $\mathcal{C}$, then a groupoid $\mathcal{C}(I, \mathbb{G})$ can be defined by setting $\mathcal{C}(I, \mathbb{G})_{0}=\mathcal{C}\left(I, G_{0}\right)$ and $\mathcal{C}(I, \mathbb{G})_{1}=\mathcal{C}\left(I, G_{1}\right)$. The groupoid structure of $\mathcal{C}(I, \mathbb{G})$ is inherited from $\mathbb{G}$, for example, the multiplication is given by $\left(y_{1}, y_{2}\right) \mapsto m\left(y_{1}, y_{2}\right)$ where $m$ is the multiplication of $\mathbb{G}$.

Proposition 11.4. Let $\mathbb{G}$ be a stably Frobenius groupoid in $\mathcal{C}$ and $I$ an object of $\mathcal{C}$. Then $\mathcal{C}(I, \mathbb{G})$ is equivalent to the full subcategory of the category of stably Frobenius adjunctions from $\mathcal{C} / I$ to $[\mathbb{G}, \mathcal{C}]$ over $\mathcal{C}$ consisting of adjunctions of the form $\mathbb{T}_{\mathbb{G}} \Sigma_{x} \dashv x^{*} U_{\mathbb{G}}$ for each $x: I \longrightarrow G_{0}$.

In other words $\mathcal{C}(I, \mathbb{G})$ embeds in the groupoid of 'stage $I$ points' of $[\mathbb{G}, \mathcal{C}]$. Do not expect the embedding to be full as there will also be the points arising from 
every $\mathbb{G}^{\prime}$ Morita equivalent to $\mathbb{G}$ in this groupoid (see C5.2.4 of [J02] for a reference to a concrete example).

Proof. Write $p_{x}$ for the adjunction $\mathbb{T}_{\mathbb{G}} \Sigma_{x} \dashv x^{*} U_{\mathbb{G}}$. The Hilsum-Skandalis map associated with $p_{x}$ is the principal $\mathbb{G}$-bundle $G_{1} \times_{G_{0}} I \longrightarrow I$ (i.e. $\pi_{2}: d_{0}^{*} x \longrightarrow I$ ) where the $\mathbb{G}$-action is given by $m \times I d_{I}$. Define a functor $F$ by $x \mapsto p_{x}$ on objects and send any morphism $y: I \longrightarrow G_{1}$ to

$$
\begin{array}{r}
G_{1} \times{G_{0}} I \longrightarrow G_{1} \times_{G_{0}} I \\
(g, i) \mapsto\left(g y(i)^{-1}, i\right)
\end{array}
$$

where the domain is a pullback of $I \stackrel{y}{\longrightarrow} G_{1} \stackrel{d_{0}}{\longrightarrow} G_{0}$ and the codomain a pullback of $I \stackrel{y}{\longrightarrow} G_{1} \stackrel{d_{1}}{\longrightarrow} G_{0}$. This defines a $\mathbb{G}$-homomorphism over $I$ and certainly by considering the groupoid identity we see that $F$, defined on morphisms in this way, is faithful. Now, given two objects $x_{1}$ and $x_{2}$ of $\mathcal{C}(I, \mathbb{G})$, a map of principal bundles $\psi: G_{1} \times_{G_{0}} I \longrightarrow G_{1} \times_{G_{0}} I$ must be of the form $\left(\theta, \pi_{2}\right)$ for some $\theta$ : $G_{1} \times G_{0} I \longrightarrow G_{1}$ as $\psi$ is over $I$. But in fact as $\psi$ is also a $\mathbb{G}$-homomorphism $\theta$ must be $(g, i) \mapsto g y(i)^{-1}$ where $y: I \longrightarrow G_{1}$ is given by $i \mapsto\left[\theta\left(e x_{1} i, i\right)\right]^{-1}$. It is easy to see that $d_{0} \theta\left(e x_{1} i, i\right)=x_{2} i$ and $d_{1} \theta\left(e x_{1} i, i\right)=x_{1} i$ and so $y$ is a morphism from $x_{1}$ to $x_{2}$. Hence $F$ is full and this completes the proof as $F$ is essentially surjective by definition of the codomain category.

\section{Hilsum-SKANDALIS MAPS INVERT ESSENTIAL EQUIVALENCES}

In this section we use the representation of Hilsum-Skandalis maps as stably Frobenius adjunctions to give a short proof that Hilsum-Skandalis maps correspond to the maps of a category that universally inverts internal essential equivalences. This result is a known characteristic of Hilsum-Skandalis maps, though has perhaps not been observed at this level of generality before. The result is also notable as it does not require the machinery of a calculus of fractions. Let $S F G p d_{\mathcal{C}}$ be the 2-category whose objects are stably Frobenius groupoids internal to $\mathcal{C}$, whose morphisms are internal functors and whose 2-cells are internal natural transformations. Let $T_{\mathcal{C}}$ be the 2-category whose objects are categories of the form $[\mathbb{G}, \mathcal{C}]$ with stably Frobenius $\mathbb{G}$, whose morphisms are stably Frobenius adjunctions over $\mathcal{C}$ and whose 2-cells are natural transformatons between the left adjoints that commute with connected components functors in the manner as described in Section 3.2

Before the statement and proof of the theorem we need a lemma:

Lemma 12.1. If $h:\left(Y_{g}, b\right) \longrightarrow\left(X_{f}, a\right)$ is a $\mathbb{G}$-homomorphism then $\left(h: Y \longrightarrow X, I d_{G_{1}} \times\right.$ $\left.h: G_{1} \times_{G_{0}} Y \longrightarrow G_{1} \times_{G_{0}} X\right)$ determines an internal functor $\mathfrak{h}: \mathbb{X}_{f, a} \longrightarrow \mathbb{Y}_{g, b}$ (i.e Lemma 7.5 extends to morphisms). The functor $\mathfrak{h}$ is an essential equivalence iff $h: Y \longrightarrow X$ is an effective descent morphism.

Proof. That an internal functor is defined is routine from definitions. For the effective descent claim, note that the relevant map is $Y \times_{X}\left(G_{1} \times_{G_{0}} X\right) \stackrel{\pi_{2}}{\longrightarrow} G_{1} \times_{G_{0}}$ $X \stackrel{\pi_{2}}{\longrightarrow} X$ where the domain is the pullback of $a$ along $h$. But this map factors as $h c$ where $c: Y \times_{X}\left(G_{1} \times_{G_{0}} X\right) \longrightarrow Y$ is $(y,(g, x)) \mapsto g^{-1} y$ since $h$ is a $\mathbb{G}$ homomorphism. As $c$ is a split epimorphism, using the groupoid identity, we can complete by recalling the remarks of Section 6 which outlined how a composition of 
two morphisms is an effective descent morphism if and only if the second factor is an effective descent morphism, provided the first factor is a split epimorphism.

Theorem 12.2. There is a pseudo-functor $\left.{ }_{-}, \mathcal{C}\right]: S F G p d_{\mathcal{C}} \longrightarrow T_{\mathcal{C}}$ which takes essential equivalences to equivalences and has the property that for any other pseudofunctor $\mathcal{N}: S F G p d_{\mathcal{C}} \longrightarrow N$ which takes essential equivalences to equivalences, there is a pseudo-functor $\overline{\mathcal{N}}: T_{\mathcal{C}} \longrightarrow N$, unique up to equivalence, such that $\overline{\mathcal{N}}[-, \mathcal{C}] \simeq \mathcal{N}$.

Proof. Part (ii) of Lemma 10.3, which can be applied as we are restricting to stably Frobenius groupoids, shows how to define $[-, \mathcal{C}]$ on morphisms; send an internal functor $\mathbb{F}: \mathbb{H} \longrightarrow \mathbb{G}$ to $\Sigma_{\mathbb{F}} \dashv \mathbb{F}^{*}$. If $\alpha: \mathbb{F}^{a} \longrightarrow \mathbb{F}^{b}$ is an internal natural transformation (where $\mathbb{F}^{a}, \mathbb{F}^{b}: \mathbb{H} \Longrightarrow \mathbb{G}$ ) then for any $\mathbb{G}$-object $\left(X_{f}, a\right)$ consider the map $\left(F_{0}^{a}\right)^{*} X_{f} \longrightarrow\left(F_{0}^{b}\right)^{*} X_{f}$ defined by $\left(\pi_{1}, a\left(\alpha \times I d_{X}\right)\right)$; this determines a natural transformation from $\left(\mathbb{F}^{a}\right)^{*}$ to $\left(\mathbb{F}^{b}\right)^{*}$ which can be seen to be a 2-cell of $T_{\mathcal{C}}$. Proposition 10.4 shows that essential equivalences are sent to equivalences.

Let us now say we are given a pseudofunctor $\mathcal{N}: S F G p d_{\mathcal{C}} \longrightarrow N$ which takes essential equivalences to equivalences. Define $\overline{\mathcal{N}}: T_{\mathcal{C}} \longrightarrow N$ on objects by $\overline{\mathcal{N}}[\mathbb{G}, \mathcal{C}]=\mathcal{N} \mathbb{G}$; this is well defined up to equivalence by Corrolary 11.2. Given a morphism $L \dashv R$ of $T_{\mathcal{C}}$, from $[\mathbb{H}, \mathcal{C}]$ to $[\mathbb{G}, \mathcal{C}]$, we know there is a Hilsum-Skandalis map $\mathfrak{P}_{L, R}$ from $\mathbb{H}$ to $\mathbb{G}$. But then there is a span $\mathbb{H} \stackrel{\mathfrak{p}}{\longrightarrow} \mathbb{P}_{L, R} \stackrel{\mathfrak{q}}{\longrightarrow} \mathbb{G}$, with $\mathfrak{p}$ an essential equivalence and so we can define $\overline{\mathcal{N}}(L \dashv R)=\mathcal{N} \mathfrak{q}(\mathcal{N} \mathfrak{p})^{-1}$. If $\alpha:\left(L_{1} \dashv R_{1}\right) \longrightarrow\left(L_{2} \dashv R_{2}\right)$ is a 2 -cell of $T_{\mathcal{C}}$ then we know (Theorem 11.3) that there is a morphism of Hilsum-Skandalis maps, $\mathfrak{P}_{L_{1}, R_{1}} \longrightarrow \mathfrak{P}_{L_{2}, R_{2}}$. So, by the lemma, $\alpha$ gives rise to an internal functor $t^{\alpha}: \mathbb{P}_{L_{1}, R_{1}} \longrightarrow \mathbb{P}_{L_{2}, R_{2}}$ which clearly must commute with the spans associated with the $L_{i} \dashv R_{i}$ s in the obvious manner (i.e. $\mathfrak{p}_{2} t^{\alpha}=\mathfrak{p}_{1}$ and $\mathfrak{q}_{2} t^{\alpha}=\mathfrak{q}_{1}$ ). Note then that because $\mathcal{N} \mathfrak{p}_{1}$ and $\mathcal{N} \mathfrak{p}_{2}$ are both equivalences (in $N$ ) so is $\mathcal{N} t^{\alpha}$. As $\mathcal{N}$ is a pseudofunctor we therefore have a 2-cell $\mathcal{N} \mathfrak{q}_{1}\left(\mathcal{N} \mathfrak{p}_{1}\right)^{-1} \longrightarrow \mathcal{N} \mathfrak{q}_{2} \mathcal{N} t^{\alpha}\left(\mathcal{N} \mathfrak{p}_{2} t^{\alpha}\right)^{-1} \longrightarrow \mathcal{N} \mathfrak{q}_{2} \mathcal{N} t^{\alpha}\left(\mathcal{N} t^{\alpha}\right)^{-1}\left(\mathcal{N} \mathfrak{p}_{2}\right)^{-1} \longrightarrow \mathcal{N} \mathfrak{q}_{2}\left(\mathcal{N} \mathfrak{p}_{2}\right)^{-1}$ This defines $\overline{\mathcal{N}}$ on 2-cells. Because of our representation of Hilsum-Skandalis as stably Frobenius adjunctions, the definition of $\overline{\mathcal{N}}$ on morphisms and 2-cells just given are unique up to equivalence given the requirement of $\mathcal{N}$ to factor as $\overline{\mathcal{N}}[-, \mathcal{C}]$.

All that remains is to check that $\overline{\mathcal{N}}$ is pseudo-functorial; i.e. if there are two stably Frobenius adjunctions

$$
[\mathbb{H}, \mathcal{C}] \underset{R_{1}}{\stackrel{L_{1}}{\rightleftarrows}}[\mathbb{G}, \mathcal{C}] \underset{R_{2}}{\stackrel{L_{2}}{\rightleftarrows}}[\mathbb{K}, \mathcal{C}]
$$

over $\mathcal{C}$ then $\mathcal{N} \mathfrak{q}_{2}\left(\mathcal{N} \mathfrak{p}_{2}\right)^{-1} \mathcal{N} \mathfrak{q}_{1}\left(\mathcal{N} \mathfrak{p}_{1}\right)^{-1}$ is isomorphic (in $\left.N\right)$ to $\mathcal{N} \mathfrak{q}_{12}\left(\mathcal{N} \mathfrak{p}_{12}\right)^{-1}$ where $\mathfrak{p}_{12}, \mathfrak{q}_{12}$ is the span associated with the composite adjunction $L_{2} L_{1} \dashv R_{1} R_{2}$. If $\mathfrak{P}_{i}=$ $\left(P_{i}, \ldots\right)$ are the Hilsum-Skandalis maps associated with $L_{i} \dashv R_{i}$, for $i=1,2$, then $P_{1} \times G_{0} P_{2}$ can be seen to be a $\mathbb{H} \times \mathbb{G} \times \mathbb{K}$-object; the action is $\left[(h, g, k),\left(x_{1}, x_{2}\right)\right] \mapsto$ $\left(h g x_{1}, k g x_{2}\right)$. We write this $\mathbb{H} \times \mathbb{G} \times \mathbb{K}$-object as $\mathfrak{P}_{123}$ with $\mathbb{P}_{123}$ the associated internal groupoid. As a $\mathbb{G}$-object, $\mathfrak{P}_{123}$ is $L_{1} \mathbb{T}_{\mathbb{H}} 1 \times R_{2} \mathbb{T}_{\mathbb{K}} 1$ and because $\Sigma_{\mathbb{G}}\left(L_{1} \mathbb{T}_{\mathbb{H}} 1 \times\right.$ $\left.R_{2} \mathbb{T}_{\mathbb{K}} 1\right) \cong \Sigma_{\mathbb{G}} L_{1}\left(\mathbb{T}_{\mathbb{H}} 1 \times R_{1} R_{2} \mathbb{T}_{\mathbb{K}} 1\right) \cong \Sigma_{\mathbb{H}}\left(\mathbb{T}_{\mathbb{H}} 1 \times R_{1} R_{2} \mathbb{T}_{\mathbb{K}} 1\right)$ the underlying object of $\Sigma_{\mathbb{G}}$ applied to $\mathfrak{P}_{123}$ is $\mathfrak{P}_{13}$, i.e. the $\mathbb{H} \times \mathbb{K}$-object associated with $L_{2} L_{1} \dashv R_{1} R_{2}$. Further it is easy to see that $\Sigma_{\mathbb{G}}$ determines a functor $\mathfrak{u}: \mathbb{P}_{123} \longrightarrow \mathbb{P}_{13}$ such that $\mathbb{P}_{123} \stackrel{\pi_{1}}{\longrightarrow} \mathbb{P}_{1} \stackrel{\mathfrak{p}_{1}}{\longrightarrow} \mathbb{H}$ factors as $\mathfrak{p}_{13} \mathfrak{u}$ and $\mathbb{P}_{123} \stackrel{\pi_{2}}{\longrightarrow} \mathbb{P}_{2} \stackrel{\mathfrak{q}_{2}}{\longrightarrow} \mathbb{K}$ factors as $\mathfrak{q}_{13} \mathfrak{u}$. Let us say that $\pi_{1}: \mathbb{P}_{123} \longrightarrow \mathbb{P}_{1}$ is an essential equivalence; then $\mathcal{N} \pi_{1}$ is an equivalence 
in $N$ and so is $\mathcal{N} \mathfrak{u}$. But then certainly $\overline{\mathcal{N}}$ is pseudo-functorial; to see this consider

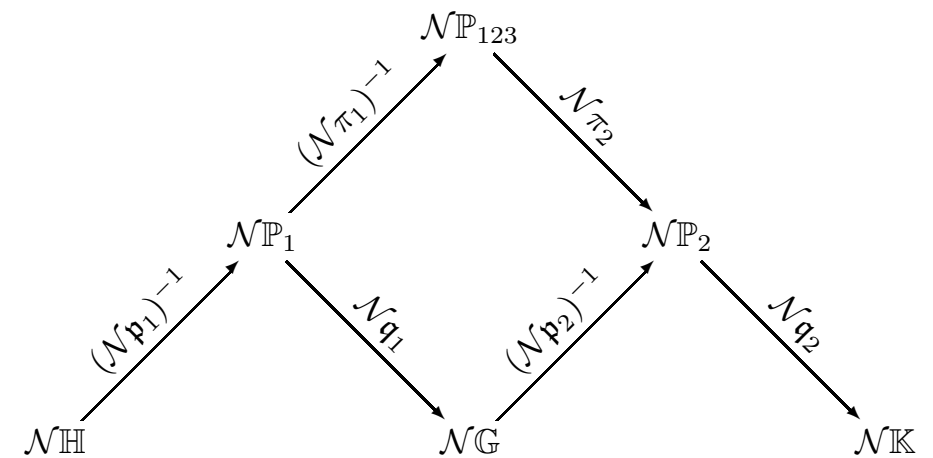

and the factorization, up to isomorphsm, of the identity on $\mathcal{N} \mathbb{P}_{13}$ as $\mathcal{N} \mathfrak{u}\left(\mathcal{N} \mathfrak{u}^{-1}\right)$ to see that $\mathcal{N} \mathfrak{q}_{2}\left(\mathcal{N} \mathfrak{p}_{2}\right)^{-1} \mathcal{N} \mathfrak{q}_{1}\left(\mathcal{N} \mathfrak{p}_{1}\right)^{-1}$ is isomorphic to $\mathcal{N} \mathfrak{q}_{12}\left(\mathcal{N} \mathfrak{p}_{12}\right)^{-1}$.

So, to complete, we must check that the projection functor $\pi_{1}: \mathbb{P}_{123} \longrightarrow \mathbb{P}_{1}$ is an essential equivalence. On objects $\pi_{1}$ is the first projection $P_{1} \times_{G_{0}} P_{2} \longrightarrow P_{1}$ which is an effective descent morphism because it is the pullback of $P_{2} \longrightarrow G_{0}$ (which is an effective descent morphism as $\mathfrak{P}_{2}$ is a Hilsum-Skandlis map). Therefore $\pi_{1}$ is essentially surjective by the lemma. To prove that $\pi_{1}$ is fully faithful we must show that $H_{1} \times_{H_{0}} G_{1} \times_{G_{0}} K_{1} \times_{K_{0}}\left(P_{1} \times_{G_{0}} P_{2}\right) \longrightarrow\left[\left(P_{1} \times_{G_{0}} P_{2}\right) \times\left(P_{1} \times_{G_{0}} P_{2}\right)\right] \times_{P_{1} \times P_{1}}$ $\left(H_{1} \times{ }_{H_{0}} G_{1} \times_{G_{0}} P_{1}\right)$ given by

$$
\left(h, g, k,\left(x_{1}, x_{2}\right)\right) \mapsto\left(\left[\left(h g x_{1}, k g x_{2}\right),\left(x_{1}, x_{2}\right)\right],\left(h, g, x_{1}\right)\right)
$$

is an isomorphsm. But as $\mathfrak{P}_{2}$ is a Hilsum-Skandalis map we have that $K_{1} \times_{K_{0}}$ $P_{2} \stackrel{\left(*, \pi_{2}\right)}{\longrightarrow} P_{2} \times_{K_{0}} P_{2}$ is an isomorphism and so the inverse is given by

$$
([(h g x, y),(x, z)],(h, g, x)) \mapsto\left(h, g, \pi_{1}\left(*, \pi_{2}\right)^{-1}(y, g z),(x, z)\right) .
$$

\section{Composing MONADiC FUnCTORS}

In order to give a categorical construction of semi-direct product using the techniques developed, which is the topic of the next section, we need a result about when monadic functors are closed under composition. It is not true in general that the composition of two monadic functors is monadic; however,

Lemma 13.1. [Hannah's lemma.] Given a diagram of adjunctions

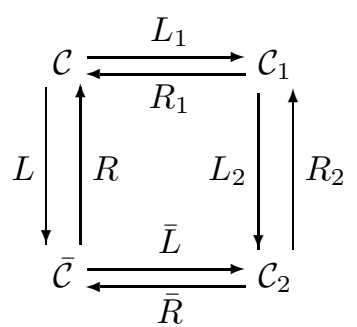

that commutes up to natural isomorphism (i.e. we are given a natural isomorphism $R_{1} R_{2} \stackrel{\cong}{\longrightarrow} \bar{R}$ ) and that satisfies Beck-Chevalley (i.e. the canonical natural transformation $L R_{1} \longrightarrow \bar{R} L_{2}$ is an isomorphism), if $R_{1}$ and $R_{2}$ are both monadic then so is $R_{1} R_{2}$. 
Proof. First note that we can assume that $\mathcal{C}_{2}$ is $\mathcal{C}_{1}^{\mathbb{T}_{2}}$ where $\mathbb{T}_{2}$ is the monad induced by $L_{2} \dashv R_{2} ; R_{2}$ is the forgetful functor. We apply Beck's theorem and as the property of reflecting isomorphisms is closed under composition what remains is a check that if $a, b: A \longrightarrow B$ is a pair of arrows in $\mathcal{C}_{2}$ that is $R_{1} R_{2}$-split then the pair has a coequalizer that is mapped under $R_{1} R_{2}$ to the split, up to canonical isomorphism. As $R_{1}$ is monadic there exists a coequalizer

$$
R_{2} A \underset{R_{2} b}{\stackrel{R_{2} a}{\longrightarrow}} R_{2} B \stackrel{q}{\longrightarrow} Q
$$

in $\mathcal{C}_{1}$ such that $R_{1} Q \cong Q_{0}$ where $Q_{0}$ is the split coequalizer of $R_{1} R_{2} a, R_{1} R_{2} b$. Now consider $R_{1} R_{2} L_{2}$ applied to $\left(^{*}\right)$. By the assumption that the diagram commutes up to isomorphism and that Beck-Chevalley holds we get,

$$
R L R_{1} R_{2} A \underset{R L R_{1} R_{2} b}{\stackrel{R L R_{1} R_{2} a}{\longrightarrow}} R L R_{1} R_{2} B \stackrel{q}{\longrightarrow} R L R_{1} Q
$$

which is part of a split coequalizer diagram as $a, b$ are $R_{1} R_{2}$-split (and split coequalizer diagrams are preserved by functors; here $R L$ ). It follows that as $R_{1}$ creates coequalizers for such split diagrams and reflects isomorphisms, that $R_{2} L_{2} q$ is the coequalizer of $R_{2} L_{2} R_{2} a, R_{2} L_{2} R_{2} b$ and in particular, by naturality of the counit $\epsilon^{2}$ of $L_{2} \dashv R_{2}$, that $R_{2} L_{2} R_{2} B \stackrel{R_{2} \epsilon_{B}^{2}}{\longrightarrow} R_{2} B \stackrel{q}{\longrightarrow} Q$ factors through $R_{2} L_{2} q$, say via $c: R_{2} L_{2} Q \longrightarrow Q$. From the definition of $c$ it is readily checked that $(Q, c)$ satisfies the unit condition required to be a $\mathbb{T}_{2}$-algebra. A similar argument to the one just deployed to show that $R_{2} L_{2} q$ is a coequalizer also shows that $R_{2} L_{2} R_{2} L_{2} q$ is the coequalizer of $R_{2} L_{2} R_{2} L_{2} R_{2} a, R_{2} L_{2} R_{2} L_{2} R_{2} b$ and so $R_{2} L_{2} R_{2} L_{2} q$ is an epimorphism. From this observation it is easy to check that $(Q, c)$ satisfies the associative condition required to be a $\mathbb{T}_{2}$-algebra and so it is a $\mathbb{T}_{2}$-algebra and from this it is clear that $q$ is the coequalizer of $a, b$.

As a consequence:

Lemma 13.2. If $X$ and $Y$ are two objects of a category $\mathcal{C}$ and both $!^{X}: X \longrightarrow 1$ and $\pi_{2}: Y \times X \longrightarrow X$ are effective descent morphisms then $!^{Y \times X}: Y \times X \longrightarrow 1$ is an effective descent morphism.

Proof. Consider the diagram of adjunctions:

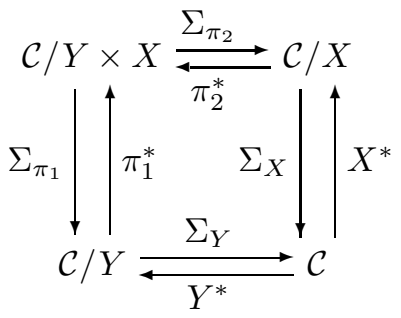

which clearly commutes and satisfies Beck-Chevalley.

\section{SEMI-DIRECT PRODUCTS}

In this section we exploit the result just given on the composition of effective descent morphisms to give a categorically flavoured description of semi-direct product. The result provides a non-trivial application of our proposition that characterises connected component adjunctions (Propositon [.3) and is a generalisation 
of Lemma 7.5 from trivial internal groupoids (i.e. from slices of $[\mathbb{G}, \mathcal{C}]$ ) to all stably Frobenius internal groupoids:

Proposition 14.1. We are given $\mathbb{G}$, a groupoid in $\mathcal{C}$ with a connected components adjunction, and $\mathfrak{K}$, a stably Frobenius groupoid in $[\mathbb{G}, \mathcal{C}]$. Then there exists a groupoid $\mathbb{G} \ltimes \mathfrak{K}$ internal to $\mathcal{C}$ and an equivalence $\Theta:[\mathbb{G} \ltimes \mathfrak{K}, \mathcal{C}] \stackrel{\simeq}{\longrightarrow}[\mathfrak{K},[\mathbb{G}, \mathcal{C}]]$ such that $\Sigma_{\mathbb{G}} \Sigma_{\mathfrak{K}} \Theta \cong \Sigma_{\mathbb{G} \ltimes \mathfrak{K}}$. Further, if $\mathbb{G}$ is stably Frobenius then so is $\mathbb{G} \ltimes \mathfrak{K}$.

Proof. The proof can be completed by showing that condition 4. of Proposition 7.3 is closed under composition. We are given stably Frobenius adjunctions $L_{0} \dashv R_{0}$ : $\mathcal{D}_{0} \rightleftarrows \mathcal{D}$ and $L \dashv R: \mathcal{D} \rightleftarrows \mathcal{C}$ where there are objects $W_{0}$ and $W$ of $\mathcal{D}_{0}$ and $\mathcal{D}$ respectively (with both $W_{0} \longrightarrow 1$ and $W \longrightarrow 1$ of effective desecent) such that $L_{W}: \mathcal{D} / W \longrightarrow \mathcal{C} / L W$ and $\left(L_{0}\right)_{W_{0}}: \mathcal{D}_{0} / W_{0} \simeq \mathcal{D} / L_{0} W_{0}$ are both equivalences. Since $\mathfrak{K}$ is stably Frobenius we can assume that $L_{0} \dashv R_{0}$ is stably Frobenius and so $\Sigma_{L_{0} W_{0}} \dashv\left(L_{0} W_{0}\right)^{*}$ factors up to isomorphism as

$$
\mathcal{D} / L_{0} W_{0} \underset{\left(L_{0}\right)_{W_{0}}}{\stackrel{\left[\left(L_{0}\right)_{W_{0}}\right]^{-1}}{\rightleftarrows}} \mathcal{D}_{0} / W_{0} \underset{W_{0}^{*}}{\stackrel{\Sigma_{W_{0}}}{\rightleftarrows}} \mathcal{D}_{0} \underset{R_{0}}{\stackrel{L_{0}}{\rightleftarrows}} \mathcal{D} .
$$

Then, by taking the slice at $W$, we can conclude that $\mathcal{D}_{0} / W_{0} \times R_{0} W$ is equivalent to $\mathcal{D} / L_{0} W_{0} \times W$. Now $\pi_{1}^{*}: \mathcal{D} / L_{0} W_{0} \longrightarrow \mathcal{D} / L_{0} W_{0} \times W$ is monadic as it is the slice at $L_{0} W_{0}$ of the monadic functor $W^{*}: \mathcal{D} \longrightarrow \mathcal{D} / W$; hence $\pi_{1}: W_{0} \times R_{0} W \longrightarrow W_{0}$ is an effective descent morphism. By Lemma 13.2 the morphism ! : $W_{0} \times R_{0} W \longrightarrow 1$ is an effective descent morphism. But this essentially completes the proof because $\mathcal{D} / L_{0} W_{0} \times W$ is a slice of $\mathcal{D} / W$ and $\mathcal{D} / W$ is (equivalent to) the slice $\mathcal{C} / L W$.

The 'Further' part is clear as stably Frobenius adjunctions are stable under composition.

Let us be explicit about the structure of $\mathbb{G} \ltimes \mathfrak{K}$. Say $\mathfrak{K}=\left(\left(K_{f_{1}}^{1}, a_{1}\right) \underset{d_{1}^{\mathfrak{\Re}}}{\stackrel{d_{0}^{\mathfrak{K}}}{\longrightarrow}}\left(K_{f_{0}}^{0}, a_{0}\right), \ldots\right)$.

In the proof of the last Proposition we have $\mathcal{D}=[\mathbb{G}, \mathcal{C}], W=\mathbb{T}_{\mathbb{G}} 1$ and $L W=\Sigma_{\mathbb{G}} W$ is $G_{0}$. Similarly $W_{0}$ is $\mathbb{T}_{\mathfrak{K}} 1, L_{0}=\Sigma_{\mathfrak{K}}, L_{0} W_{0}$ is $\left(K_{f_{0}}^{0}, a_{0}\right)$ and $L_{0}\left(W_{0} \times W_{0}\right)$ is $\left(K_{f_{1}}^{1}, a_{1}\right)$. The object of objects of $\mathbb{G} \ltimes \mathfrak{K}$, constructed in the proof of the Proposition, is $\Sigma_{\mathbb{G}}$ applied to the image of 1 under the equivalences $\mathcal{D}_{0} / W_{0} \times$ $R_{0} W \longrightarrow \mathcal{D} / L_{0} W_{0} \times W$ which is $\Sigma_{\mathbb{G}}\left(L_{0} W_{0} \times \mathbb{T}_{\mathbb{G}} 1\right)$ which from earlier identities we know is $\Sigma_{G_{0}} U_{\mathbb{G}}\left(L_{0} W_{0}\right)=K^{0}$. Now Proposition 7.3 shows us that $d_{0}^{\mathfrak{K}}=L_{0} \pi_{2}$ and $d_{1}^{\mathfrak{K}}=L_{0} \pi_{1}$ where $L_{0} \pi_{i}: L_{0}\left(W_{0} \times W_{0}\right) \longrightarrow L_{0} W_{0}$. The domain and codomain maps of $\mathbb{G} \ltimes \mathfrak{K}$ are $\Sigma_{\mathbb{G}} L_{0} \pi_{i}: \Sigma_{\mathbb{G}} L_{0}\left(\left[W_{0} \times R_{0} W\right] \times\left[W_{0} \times R_{0} W\right]\right) \longrightarrow \Sigma_{\mathbb{G}} L_{0}\left(\left[W_{0} \times R_{0} W\right]\right)$ for $i=2,1$ respectively, which by application of the Frobenius reciprocity assumption on $L_{0} \dashv R_{0}$ are $\Sigma_{\mathbb{G}}\left(\pi_{i} \times L_{0} \pi_{i}\right): \Sigma_{\mathbb{G}}\left(\left[\mathbb{T}_{\mathbb{G}} 1 \times \mathbb{T}_{\mathbb{G}} 1\right] \times L_{0}\left[W_{0} \times W_{0}\right]\right) \longrightarrow \Sigma_{\mathbb{G}}\left(\mathbb{T}_{\mathbb{G}} 1 \times\right.$ $\left.L_{0} W_{0}\right)$. But, see the diagram before Proposition 7.3 the image of the two projections $\pi_{2}$ and $\pi_{1}$ onto $\mathbb{T}_{\mathbb{G}} 1$ under the connected components functor of $\mathbb{G}$ can be identified with the projection and $\mathbb{G}$-action respectively and so the domain and codomain maps of $\mathbb{G} \ltimes \mathfrak{K}$ can be seen to be $G_{1} \times{ }_{G_{0}} K^{1} \stackrel{\pi_{2}}{\longrightarrow} K^{1} \stackrel{d_{0}^{\mathfrak{K}}}{\longrightarrow} K^{0}$ and $G_{1} \times_{G_{0}} K^{1} \stackrel{a_{1}}{\longrightarrow} K^{1} \stackrel{d_{1}^{\mathfrak{R}}}{\longrightarrow} K^{0}$.

As for the multiplication we first pause for a lemma:

Lemma 14.2. If $\mathbb{G}$ is a groupoid with a connected components adjunction and $\left(X_{f}, a\right)$ a $\mathbb{G}$-object, then under the canonical isomorphisms the morphisms $\Sigma_{\mathbb{G}}\left(\pi_{i j} \times\right.$ 


$$
\begin{aligned}
& I d): \Sigma_{\mathbb{G}}\left(\mathbb{T}_{\mathbb{G}} 1 \times \mathbb{T}_{\mathbb{G}} 1 \times \mathbb{T}_{\mathbb{G}} 1 \times\left(X_{f}, a\right)\right) \longrightarrow \Sigma_{\mathbb{G}}\left(\mathbb{T}_{\mathbb{G}} 1 \times \mathbb{T}_{\mathbb{G}} 1 \times\left(X_{f}, a\right)\right) \text { are } \\
& G_{1} \times_{G_{0}} G_{1} \times G_{0} X \longrightarrow G_{1} \times_{G_{0}} X \\
& \left(g_{1}, g_{2}, x\right) \quad \mapsto \quad\left(g_{1}, g_{2} x\right) \\
& \left(g_{1}, g_{2}, x\right) \quad \mapsto \quad\left(g_{1} g_{2}, x\right) \\
& \left(g_{1}, g_{2}, x\right) \quad \mapsto \quad\left(g_{2}, x\right)
\end{aligned}
$$

for $i j=12,13,23$ respectively.

Proof. Apply $\mathbb{T}_{\mathbb{G}} 1 \times(-)$ to the diagrams just before the start of subsection 7.2 For any $\mathbb{G}$-homomorphism $h, I d_{\mathbb{T}_{\mathbb{G}} 1} \times h$ is canonically isomorphic to $\mathbb{T}_{\mathbb{G}} U_{\mathbb{G}} h$ by naturality of $\epsilon$. Applying to $h=\mathbb{T}_{\mathbb{G}} U_{\mathbb{G}} \epsilon_{\left(X_{f}, a\right)}$ and $h=\epsilon_{\mathbb{T}_{\mathbb{G}} U_{\mathbb{G}}\left(X_{f}, a\right)}$ we see that for $i j=12,13$ respectively, $\Sigma_{\mathbb{G}}\left(\pi_{i j} \times I d\right)$ is canonically isomorphic to $U_{\mathbb{G}} \mathbb{T}_{\mathbb{G}} U_{\mathbb{G}} \epsilon_{\left(X_{f}, a\right)}$ and $U_{\mathbb{G}} \epsilon_{\mathbb{T}_{\mathbb{G}} U_{\mathbb{G}}\left(X_{f}, a\right)}$ respectively from which these cases are clear given our knowledge of $\epsilon$.

For the $i j=23$ case apply the second diagram to the $\mathbb{G}$-object $\mathbb{T}_{\mathbb{G}} 1 \times\left(X_{f}, a\right)$ and note that $\epsilon_{\mathbb{T}_{G} U_{\mathbb{G}}\left(\mathbb{T}_{\mathbb{G}} 1 \times\left(X_{f}, a\right)\right)}$ is canonically isomorphic to $\epsilon_{\mathbb{T}_{G}} U_{\mathbb{G}} \mathbb{T}_{\mathbb{G}} U_{\mathbb{G}}\left(X_{f}, a\right)$. We have commented in the paragraphs leading up to Proposition 7.3 that $\Sigma_{\mathbb{G}}$ applied to the counit at the free algebra returns the relevant projection.

Returning to multiplication, the 4. implies 1. part of Proposition 7.3 shows that it is

$L_{0}\left(W_{0} \times W_{0}\right) \times_{L_{0} W_{0}} L_{0}\left(W_{0} \times W_{0}\right) \stackrel{\left(L_{0} \pi_{12}, L_{0} \pi_{23}\right)^{-1}}{\longrightarrow} L_{0}\left(W_{0} \times W_{0} \times W_{0}\right) \stackrel{L_{0} \pi_{13}}{\longrightarrow} L_{0}\left(W_{0} \times W_{0}\right)$

for $\mathfrak{K}$ and similarly for $\mathbb{G}$ and $\mathbb{G} \ltimes \mathfrak{K}$. So, again exploiting the fact that $L_{0} \dashv R_{0}$ satisfies Frobenius reciprocity, the multiplication of $\mathbb{G} \ltimes \mathfrak{K}$ is the composition of $\Sigma_{\mathbb{G}}\left(\pi_{13} \times L_{0} \pi_{13}\right)$

$\Sigma_{\mathbb{G}}\left(\mathbb{T}_{\mathbb{G}} 1 \times \mathbb{T}_{\mathbb{G}} 1 \times \mathbb{T}_{\mathbb{G}} 1 \times L_{0}\left(W_{0} \times W_{0} \times W_{0}\right)\right) \longrightarrow \Sigma_{\mathbb{G}}\left(\mathbb{T}_{\mathbb{G}} 1 \times \mathbb{T}_{\mathbb{G}} 1 \times L_{0}\left(W_{0} \times W_{0}\right)\right)$

and $\left[\Sigma_{\mathbb{G}}\left(\pi_{12}, L_{0} \pi_{12}\right), \Sigma_{\mathbb{G}}\left(\pi_{23}, L_{0} \pi_{23}\right)\right]^{-1}$

$$
\begin{aligned}
\Sigma_{\mathbb{G}}\left(\mathbb{T}_{\mathbb{G}} 1 \times \mathbb{T}_{\mathbb{G}} 1 \times L_{0}\left(W_{0} \times W_{0}\right)\right) \times \Sigma_{\Sigma_{\mathbb{G}}\left(\mathbb{T}_{\mathbb{G}} 1 \times L_{0} W_{0}\right)} \Sigma_{\mathbb{G}}\left(\mathbb{T}_{\mathbb{G}} 1 \times \mathbb{T}_{\mathbb{G}} 1 \times L_{0}\left(W_{0} \times W_{0}\right)\right) & \longrightarrow \Sigma_{\mathbb{G}}\left(\mathbb{T}_{\mathbb{G}} 1 \times \mathbb{T}_{\mathbb{G}} 1 \times \mathbb{T}_{\mathbb{G}} 1 \times L_{0}\left(W_{0} \times W_{0} \times W_{0}\right)\right) \quad(\mathrm{b})
\end{aligned}
$$

Apply the lemma to see that (a) is sending, under the canonical isomorphsms, $\left(g_{1}, g_{2},\left[k_{1}, k_{2}\right]\right)$ to $\left(g_{1} g_{2}, k_{1} k_{2}\right)$ and the inverse of $(\mathrm{b})$, i.e. $\left[\Sigma_{\mathbb{G}}\left(\pi_{12}, L_{0} \pi_{12}\right), \Sigma_{\mathbb{G}}\left(\pi_{23}, L_{0} \pi_{23}\right)\right]$, sends $\left(g_{1}, g_{2},\left[k_{1}, k_{2}\right]\right)$ to $\left(\left(g_{1}, g_{2} k_{1}\right),\left(g_{2}, k_{2}\right)\right)$ where we are using [-, ] for $\left(L_{0} \pi_{12}, L_{0} \pi_{23}\right)^{-1}$. From this it is clear the multiplcation of $\mathbb{G} \ltimes \mathfrak{K}$ is $\left((g, k),\left(g^{\prime}, k^{\prime}\right)\right) \mapsto\left(g g^{\prime},\left(\left(g^{\prime}\right)^{-1} k\right) k^{\prime}\right)$ and we have indeed defined semidirect product in the usual manner.

Going in the other direction to the last Proposition, stably Frobenius adjunctions with categories of $\mathbb{H}$-objects as domains give rise to internal groupoids:

Proposition 14.3. Let $\mathbb{H}$ be a groupoid in $\mathcal{C}$ with a connected components adjunction, $\mathcal{D} \rightleftarrows \mathcal{C}$ an adjunction and $L \dashv R:[\mathbb{H}, \mathcal{C}] \rightleftarrows \mathcal{D}$ a stably Frobenius adjunction over $\mathcal{C}$. Then there exists a stably Frobenius groupoid $\mathfrak{K}_{L, R}$ in $\mathcal{D}$ and an equivalence $\Theta:[\mathbb{H}, \mathcal{C}] \longrightarrow\left[\mathfrak{K}_{L, R}, \mathcal{D}\right]$ over $\mathcal{D}$.

Proof. By Proposition 7.3 using the second condition, we need to find an object $W$ of $\mathcal{D}$ and a stably Frobenius adjunction $\mathcal{D} / W \rightleftarrows[\mathbb{H}, \mathcal{C}]$ over $\mathcal{D}$ whose right adjoint is monadic. But by Proposition 7.3 we know that there is a stably Frobenius adjunction $\mathbb{T}_{\mathbb{H}} \dashv U_{\mathbb{H}}: \mathcal{C} / H_{0} \rightleftarrows[\mathbb{H}, \mathcal{C}]$ over $\mathcal{C}$ whose right adjoint is monadic, so to 
progress the proof we exhibit an equivalence $\mathcal{C} / H_{0} \simeq \mathcal{D} / W$. Take $W=L \mathbb{T}_{\mathbb{H}} 1$ and notice that Lemma 4.2 part 2 can be applied as $L \mathbb{T}_{\mathbb{H}} \dashv U_{\mathbb{H}} R$ is stably Frobenius because $L \dashv R$ is stably Frobenius.

When $\mathcal{D}=[\mathbb{G}, \mathcal{C}]$, for $\mathbb{G}$ stably Frobenius, describing $\mathfrak{K}_{L, R}$ is easy; it is given by $\left(L \pi_{2}, L \pi_{1}: L\left(\mathbb{T}_{\mathbb{H}} 1 \times \mathbb{T}_{\mathbb{H}} 1\right) \Longrightarrow L \mathbb{T}_{\mathbb{H}} 1, \ldots\right)$ and $L\left(\mathbb{T}_{\mathbb{H}} 1 \times \mathbb{T}_{\mathbb{H}} 1\right)$ is the $\left(R_{r}, e\right)$ that played a role in the proof of Propositin 8.3, so the domain and codomain maps of $\mathfrak{K}_{L, R}$ are canonically isomorphic to $\pi_{2}, c: H_{1} \times{ }_{H_{0}} P \Longrightarrow P$ where, as before, $\left(P_{p}, c: H_{1} \times_{H_{0}} P \longrightarrow P\right)$ is defined as $R \mathbb{T}_{\mathbb{G}} 1$.

Lemma 14.4. Given $L \dashv R$ as in the last proposition, with $\mathcal{D}=[\mathbb{G}, \mathcal{C}]$. Then the groupoid $\mathbb{G} \ltimes \mathfrak{K}_{L, R}$ in $\mathcal{C}$ is isomorphic to $\mathbb{P}_{L, R}$, the groupoid corresponding to the Hilsum-Skandalis map $\mathfrak{P}_{L, R}$

Proof. Follows from the explicit descriptions of semidirect product and $\mathfrak{K}_{L, R}$ already provided.

So, in summary, we see that the stably Frobenius adjunctions corresponding to Hilsum-Skandalis maps are the connected component adjunctions of internal stably Frobenius groupoids. Hilsum-Skandalis maps give rise to stably Frobenius groupoids internal to $[\mathbb{G}, \mathcal{C}]$ and vice versa, but these internal groupoids are unique only up to Morita equivalence.

\section{Pullback of Connected Component adjunctions}

In this section we show that the 2-category $T_{\mathcal{C}}$ defined in the introductory paragraph of Section 12 has finite pseudo-limits. It clearly has a terminal object as the trivial groupoid $\mathbb{G}=1$ is stably Frobenius. The next proposition shows that $T_{\mathcal{C}}$ has binary pseudo-products, but this is sufficient as we can exploit the results of the previous section to show that a pullback diagram over $[\mathbb{G}, \mathcal{C}]$ gives rise to a product diagram in $T_{[\mathbb{G}, \mathcal{C}]}$. We will see later that this allows us to conclude that the pullbacks of bounded geometric morphisms over some base topos $\mathcal{S}$ can be calculated in $T_{\mathbf{L o c}_{\mathcal{S}}}$, where $\mathbf{L o c}_{\mathcal{S}}$ is the category of locales over $\mathcal{S}$.

We need a lemma.

Lemma 15.1. If $\mathbb{H}$ and $\mathbb{G}$ are two groupoids in $\mathcal{C}$ then $\mathbb{T}_{\mathbb{H} \times \mathbb{G}} 1 \stackrel{\left(I d_{H_{1}} \times d_{1}^{\mathbb{G}}, d_{1}^{\mathbb{H}} \times I d_{G_{1}}\right)}{\longrightarrow} \pi_{1}^{*} \mathbb{T}_{\mathbb{H}} 1 \times$ $\pi_{2}^{*} \mathbb{T}_{\mathbb{G}} 1$ is an $\mathbb{H} \times \mathbb{G}$-isomorphism. If, further, both $\mathbb{H}$ and $\mathbb{G}$ are stably Frobenius then

(a) for any $\mathbb{H} \times \mathbb{G}$-object $A=\left(A_{f, g}, c, d\right)$, the map $n_{A}: A \longrightarrow \Sigma_{\pi_{1}} A$, which quotients out the $\mathbb{G}$-action, is an $\mathbb{H}$-homomorphism,

(b) $I d \times d_{1}^{\mathbb{G}}: \mathbb{T}_{\mathbb{H} \times \mathbb{G}} 1 \longrightarrow \pi_{1}^{*} \mathbb{T}_{\mathbb{H}} 1$ is the adjoint transpose across $\Sigma_{\pi_{1}} \dashv \pi_{1}^{*}$ of the projection $\pi_{1}: \mathbb{T}_{\mathbb{H}} 1 \times \mathbb{H}^{*} G_{0} \longrightarrow \mathbb{T}_{\mathbb{H}} 1$,

(c) for any $\mathbb{H}$-object $B=\left(B_{f}, c\right)$, the morphism

$$
\Sigma_{\mathbb{H}} \Sigma_{\pi_{1}}\left(\mathbb{T}_{\mathbb{H} \times \mathbb{G}} 1 \times \pi_{1}^{*} B\right) \stackrel{\Sigma_{\mathbb{H}}(\cong)}{\longrightarrow} \Sigma_{\mathbb{H}}\left(\Sigma_{\pi_{1}} \mathbb{T}_{\mathbb{H} \times \mathbb{G}} 1 \times B\right) \stackrel{\Sigma_{\mathbb{H}}\left(\pi_{1} \times I d\right)}{\longrightarrow} \Sigma_{\mathbb{H}}\left(\mathbb{T}_{\mathbb{H}} 1 \times B\right)
$$

is canonically isomorphic to $\pi_{1}: B \times G_{0} \longrightarrow B$; and,

(d) the morphism

$\Sigma_{\mathbb{H} \times \mathbb{G}}\left(\mathbb{T}_{\mathbb{H} \times \mathbb{G}} 1 \times A\right) \stackrel{\Sigma_{\mathbb{H} \times \mathbb{G}}\left(\left(I d \times d_{1}^{\mathfrak{G}}\right) \times I d\right)}{\longrightarrow} \Sigma_{\mathbb{H} \times \mathbb{G}}\left(\pi_{1}^{*} \mathbb{T}_{\mathbb{H}} 1 \times A\right) \stackrel{\cong}{\longrightarrow} \Sigma_{\mathbb{H}}\left(\mathbb{T}_{\mathbb{H}} 1 \times \Sigma_{\pi_{1}} A\right)$

is canonically isomorphic to $A \stackrel{n_{A}}{\longrightarrow} \Sigma_{\pi_{1}} A$. 
The functor $\Sigma_{\pi_{1}}:[\mathbb{H} \times \mathbb{G}, \mathcal{C}] \longrightarrow[\mathbb{H}, \mathcal{C}]$ used in the statement of the lemma exists by application of Proposition 10.3 .

Proof. That the given morphism is an isomorphism is clear from the construction of product in $[\mathbb{H} \times \mathbb{G}, \mathcal{C}]$; the pullback of $I d_{H_{1}} \times d_{1}^{\mathbb{G}}$ along $d_{1}^{\mathbb{H}} \times I d_{G_{1}}$ is $d_{1}^{\mathbb{H}} \times d_{1}^{\mathbb{G}}$ : $H_{1} \times G_{1} \longrightarrow H_{0} \times G_{0}$, i.e. $d_{1}^{\mathbb{H} \times \mathbb{G}}$.

Part (a) is clear from the construction of the $\mathbb{H}$-action on $\Sigma_{\pi_{1}} A$ from which the identification of $\Sigma_{\pi_{1}} \mathbb{T}_{\mathbb{H} \times \mathbb{G}} 1$ with $\mathbb{T}_{\mathbb{H}} 1 \times \mathbb{H}^{*} G_{0}$ is also clear; quotienting out the $\mathbb{G}$-action on $G_{1}$ returns $G_{0}$ and from this (b) follows as the quotienting map is $d_{1}^{\mathbb{G}}$.

For (c), first note that $\Sigma_{\pi_{1}}\left(\mathbb{T}_{\mathbb{H} \times \mathbb{G}} 1 \times \pi_{1}^{*} B\right)$ is constructed using the quotient $\left(I d \times d_{1}^{\mathbb{G}}\right) \times(I d \times I d):\left(H_{1} \times G_{1}\right) \times{ }_{H_{0} \times G_{0}}\left(B \times G_{0}\right) \longrightarrow\left(H_{1} \times G_{0}\right) \times H_{0} \times G_{0}\left(B \times G_{0}\right)$. But (c) then follows as

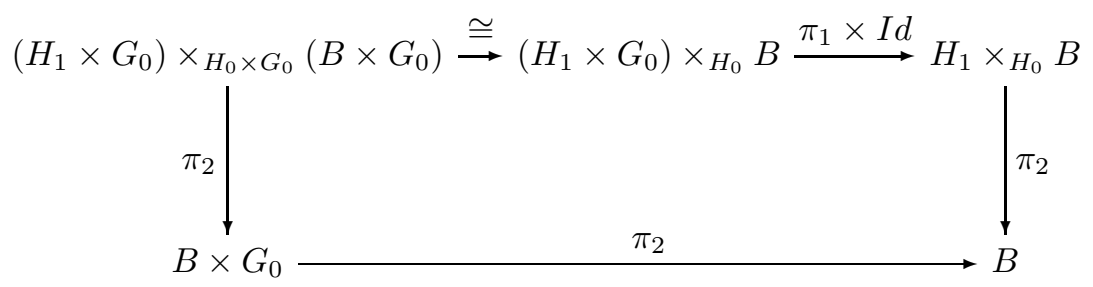

clearly commutes where the vertical arrows quotient out the $\mathbb{H}$-action.

The morphism of $(\mathrm{d})$ is $\Sigma_{\mathbb{H}}$ applied to

$$
\Sigma_{\pi_{1}}\left(\mathbb{T}_{\mathbb{H} \times \mathbb{G}} 1 \times A\right) \stackrel{\Sigma_{\pi_{1}}\left(\tilde{\pi_{1}} \times I d\right)}{\longrightarrow} \Sigma_{\pi_{1}}\left(\pi_{1}^{*} \mathbb{T}_{\mathbb{H}} 1 \times A\right) \stackrel{\cong}{\longrightarrow} \mathbb{T}_{\mathbb{H}} 1 \times \Sigma_{\pi_{1}} A
$$

where ( $\tilde{-})$ is adjoint transpose across $\Sigma_{\pi_{1}} \dashv \pi_{1}^{*}$. As the canonical isomorphism in the definition of Frobenius reciprocity involves a counit this morphism is equal to

$$
\begin{aligned}
& \Sigma_{\pi_{1}}\left(\mathbb{T}_{\mathbb{H} \times \mathbb{G}} 1 \times A\right) \stackrel{\Sigma_{\pi_{1}}\left(I d \times \eta_{A}\right)}{\longrightarrow} \quad \Sigma_{\pi_{1}}\left(\mathbb{T}_{\mathbb{H} \times \mathbb{G}} 1 \times \pi_{1}^{*} \Sigma_{\pi_{1}} A\right) \stackrel{\cong}{\longrightarrow} \Sigma_{\pi_{1}} \mathbb{T}_{\mathbb{H} \times \mathbb{G}} 1 \times \Sigma_{\pi_{1}} A \\
& \stackrel{\pi_{1} \times I d}{\longrightarrow} \mathbb{T}_{\mathbb{H}} 1 \times \Sigma_{\pi_{1}} A .
\end{aligned}
$$

The result then follows by taking $B=\Sigma_{\pi_{1}} A$ in (c) since the unit $\eta_{A}$ is the map $A \stackrel{\left(n_{A}, g\right)}{\longrightarrow} \Sigma_{\pi_{1}} A \times G_{0}$.

Proposition 15.2. $T_{\mathcal{C}}$ has binary pseudo-products.

Proof. Let $\mathbb{G}$ and $\mathbb{H}$ be two stably Frobenius groupoids in $\mathcal{C}$. Then, Proposition 10.3. $\mathbb{G} \times \mathbb{H}$ is stably Frobenius and the top horizontal and left vertical adjunctions of the diagram

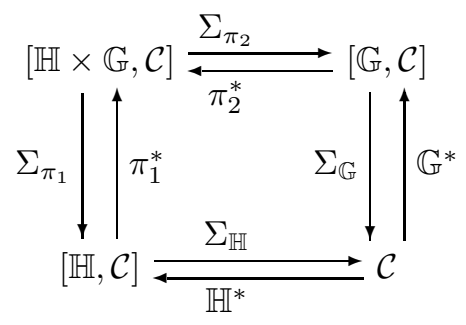

are morphisms of $T_{\mathcal{C}}$. The diagram commutes up to canonical natural isomorphism. 
For any stably Frobenius groupoid $\mathbb{K}$ given a diagram

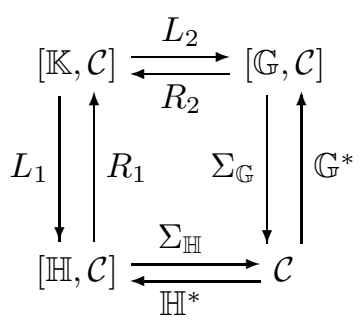

commuting up to natural isomorphism and over $\mathcal{C}$, we must construct an adjunction $[\mathbb{K}, \mathcal{C}] \underset{R}{\stackrel{L}{\rightleftarrows}}[\mathbb{H} \times \mathbb{G}, \mathcal{C}]$, unique up to natural isomorphism, such that $\Sigma_{\pi_{1}} L \cong L_{1}$ and $\Sigma_{\pi_{2}} L \cong L_{2}$. Write $\mathfrak{P}_{i}=\left(\left(P_{i}\right)_{\left(p_{i}, q_{i}\right)}, c_{i}, d_{i}\right)$ for the Hilsum-Skandalis maps corresponding to $L_{i} \dashv R_{i}$, for $i=1,2$. Notice that $P_{1} \times_{K_{0}} P_{2} \stackrel{q_{1} \times q_{2}}{\longrightarrow} H_{0} \times G_{0}$ can be made into a $\mathbb{H} \times \mathbb{G}$-object $\left(\left[(h, g),\left(x_{1}, x_{2}\right)\right] \mapsto\left(h x_{1}, g x_{2}\right)\right)$ and $p_{1} \pi_{1}: P_{1} \times_{K_{0}}$ $P_{2} \longrightarrow K_{0}$ into a $\mathbb{K}$-object $\left(\left(k,\left(x_{1}, x_{2}\right)\right) \mapsto\left(k x_{1}, k x_{2}\right)\right)$. Given that $p_{1} \pi_{1}: P_{1} \times_{K_{0}}$ $P_{2} \longrightarrow K_{0}$ is an effective descent morphism (Lemma 13.2, applied in $\mathcal{C} / K_{0}$ ) it is then easy to check that a Hilsum-Skandalis map, $\mathfrak{P}$, has been defined and so there is a corresponding adjunction $L \dashv R$. If $\left(Y_{g}, b\right)$ is a $\mathbb{K}$-object then $L_{1}\left(Y_{g}, b\right)$ is obtained by quotienting the $\mathbb{K}$-action of the $\mathbb{K} \times \mathbb{H}$-object $P_{1} \times_{K_{0}} Y$ (the action map is $((k, h), x, y) \mapsto(k h x, k y))$. Similarly $L_{\mathfrak{P}}\left(Y_{g}, b\right)$ has underlying object $\Sigma_{\mathbb{K}}\left(\left(P_{1} \times_{K_{0}}\right.\right.$ $\left.\left.P_{2}\right) \times_{K_{0}} Y\right)$. Then, since $\Sigma_{\pi_{1}}$ quotients out the $\mathbb{G}$-action, the process of quotienting commutes (the groupoids are stably Frobenius) and $K_{0} \cong \Sigma_{\mathbb{G}}\left(\left(P_{2}\right)_{q_{2}}, d_{2}\right)$, it follows that $L_{1} \cong \Sigma_{\pi_{1}} L_{\mathfrak{P}}$. Similarly $L_{2} \cong \Sigma_{\pi_{2}} L_{\mathfrak{P}}$.

To complete we must show that any adjunction $[\mathbb{K}, \mathcal{C}] \underset{R}{\stackrel{L}{\rightleftarrows}}[\mathbb{H} \times \mathbb{G}, \mathcal{C}]$ over $\mathcal{C}$ is isomorphic to $L_{\mathfrak{P}} \dashv R_{\mathfrak{P}}$ where $\mathfrak{P}$ is the Hilsum-Skandalis map whose underlying object is $P_{1} \times_{K_{0}} P_{2}$, constructed from $\Sigma_{\pi_{1}} L \dashv R \pi_{1}^{*}$ and $\Sigma_{\pi_{2}} L \dashv R \pi_{2}^{*}$ as in the last paragraph. The lemma shows that $\mathbb{T}_{\mathbb{G} \times \mathbb{H}} 1 \cong \pi_{1}^{*} \mathbb{T}_{\mathbb{H}} 1 \times \pi_{2}^{*} \mathbb{T}_{\mathbb{G}} 1$; by applying $R$ we see that the $\mathbb{K}$-object $R \mathbb{T}_{\mathbb{H} \times \mathbb{G}} 1$ is isomorphic to $P_{1} \times_{K_{0}} P_{2}$. The result then follows by taking $A=L \mathbb{T}_{\mathbb{K}} 1$ in the lemma as it shows that $R\left(I d_{H_{1}} \times d_{1}^{\mathbb{G}}\right): R \mathbb{T}_{\mathbb{H} \times \mathbb{G}} 1 \longrightarrow P_{1}$ is an $\mathbb{H}$-homomorphism (it corresponds to $n_{L \mathbb{T}_{\mathrm{K}} 1}$ under the canonical isomorphisms) and similarly $R\left(d_{1}^{\mathbb{H}} \times I d_{G_{1}}\right): R \mathbb{T}_{\mathbb{H} \times \mathbb{G}} 1 \longrightarrow P_{2}$ is a $\mathbb{G}$-homomorphism; but then by construction of $P_{1} \times K_{0} P_{2}$ as an $\mathbb{H} \times \mathbb{G}$-object the morphism $\left(R\left(I d_{H_{1}} \times d_{1}^{\mathbb{G}}\right), R\left(d_{1}^{\mathbb{H}} \times\right.\right.$ $\left.\left.I d_{G_{1}}\right)\right)$ must be an $\mathbb{H} \times \mathbb{G}$-homomorphism and so $\mathfrak{P} \cong \mathfrak{P}_{L, R}$.

\section{Geometric Morphisms as stably Frobenius adjunctions}

In this section we recall the main result of [T10, which is a representation theorem of geometric morphsms as stably Frobenius adjunctions, and give a correct account of the morphisms in the representation theorem. Familiarity with locale theory and topos theory is assumed; e.g. [J02] or [JT84]. Our toposes are elementary unless stated otherwise. We write $f: \mathcal{F} \longrightarrow \mathcal{E}$ for a geometric morphism between toposes and $\mathbf{L o c}_{\mathcal{E}}$ for the category of locales relative to a topos $\mathcal{E}$. If $X$ is a locale in $\mathcal{F}$ we write $\mathcal{O}_{\mathcal{F}} X$ for its frame of opens. The direct image $f_{*}$ of any geometric morphisms preserves frames; i.e. $f_{*} \mathcal{O}_{\mathcal{F}} X=\mathcal{O}_{\mathcal{E}} \Sigma_{f} X$ for some locale $\Sigma_{f} X$ in $\mathcal{E}$. This determines a functor $\Sigma_{f}: \operatorname{Loc}_{\mathcal{F}} \longrightarrow \operatorname{Loc}_{\mathcal{E}}$ which can be shown to have a right adjoint which, abusing notation, is written $f^{*}: \mathbf{L o c}_{\mathcal{E}} \longrightarrow \mathbf{L o c}_{\mathcal{F}}$. The abuse is reasonable because $f^{*}$ is the inverse image of $f$ when restricted to discrete locales. 
We call $\Sigma_{f} \dashv f^{*}$ the pullback adjunction associated with the geometric morphism $f$. This is reasonable because if $f$ is a geometric morphism between categories of sheaves over two locales, i.e. $f: S h(X) \longrightarrow S h(Y)$, then it is uniquely determined by a locale map $f: X \longrightarrow Y$ and under the equivalences $\operatorname{Loc}_{S h(Z)} \simeq \operatorname{Loc} / Z$, for $Z=X, Y$, the adjunction $\Sigma_{f} \dashv f^{*}$ is the pullback adjunction in the usual sense (i.e. $\left.\Sigma_{f}\left(Y_{g}\right)=Y_{f g}\right)$.

The following result is clear from T10:

Proposition 16.1. If $f: \mathcal{F} \longrightarrow \mathcal{E}$ is a geometric morphism between elementary toposes then the adjunction $\Sigma_{f} \dashv f^{*}: \mathbf{L o c}_{\mathcal{F}} \rightleftarrows \mathbf{L o c}_{\mathcal{E}}$ is stably Frobenius and $f^{*} \mathbb{S}_{\mathcal{E}} \cong \mathbb{S}_{\mathcal{F}}$. Further, for every stably Frobenius adjunction $L \dashv R$ : $\mathbf{L o c}_{\mathcal{F}} \rightleftarrows \operatorname{Loc}_{\mathcal{E}}$, if $R$ preserves $\mathbb{S}$ then there exists a geometric morphism $f$ : $\mathcal{F} \longrightarrow \mathcal{E}$, unique up natural isomorphism, such that $R \cong f^{*}$.

Here $\mathbb{S}$ is the Sierpiński locale. Its frame of opens is the feee frame on the singleton set 1. It is an internal distributive lattice in Loc and when we are asserting that it is preserved by a functor we mean that the implied isomorphism also commutes with the lattice structure.

Proof. The proof is in [T10]. Two comments are useful. 1. The proof that $\Sigma_{f} \dashv f^{*}$ is Frobenius in fact is already clear from Proposition 2(4) of Ch. 6, $§ 1$ of [JT84, and from Proposition 2 of $\S 3$ of the same Chapter, which establishes $\operatorname{Loc}_{S h(Z)} \simeq \operatorname{Loc} / Z$, it can be seen that the adjunction $\Sigma_{f} \dashv f^{*}$ is stably Frobenius (consider Proposition $4 \S 1$ ). 2. The category of locales is order enriched and $X \times \mathbb{S}$ is the tensor $X \otimes\{0 \leq 1\}$ for any locale $X$; from this it is clear that a functor between categories of locales preserves the order enrichment provided it preserves the Sierpiński locale.

To turn this last proposition into a categorical equivalence, one needs to restrict to natural isomorphisms between geometric morphisms and adjunctions. This is because the mapping $f \mapsto\left(\Sigma_{f} \dashv f^{*}\right)$ is not functorial on natural transformations; if $\alpha:\left(f_{1}\right)_{*} \longrightarrow\left(f_{2}\right)_{*}$ is a natural transformation between direct images then the morphism $\alpha_{\Omega_{\mathcal{F}} X}$ is not necessarily a frame homomorphism. But it will be if $\alpha$ is an isomorphism (use the naturality of $\alpha$ to show that $\alpha_{\Omega_{\mathcal{F}} X}$ is monotone).

However we can account for natural transformations between geometric morphisms in this representation theorem if required. To see this recall that such natural transformations can be represented as $\mathbb{S}$-homotopies. If $f, g: \mathcal{F} \Longrightarrow \mathcal{E}$ are two geometric morphisms then a $\mathbb{S}$-homotopy from $f$ to $g$ consists of a geometric morphism $H: S h_{\mathcal{F}} \mathbb{S}_{\mathcal{F}} \longrightarrow \mathcal{E}$ such that $f=H 0_{\mathbb{S}_{\mathcal{F}}}$ and $g=H 1_{\mathbb{S}_{\mathcal{F}}}$ where $0_{\mathbb{S}_{\mathcal{F}}}$ and $1_{\mathbb{S}_{\mathcal{F}}}$ are the geometric morphisms corresponding to the locale maps that are the bottom and top points of the Sierpiński locale $\mathbb{S}_{\mathcal{F}}$. The topos $S h_{\mathcal{F}} \mathbb{S}_{\mathcal{F}}$ can be described explicitly, it is the presheaf category $[\mathbf{2}, \mathcal{F}]$, where $\mathbf{2}$ is the category $(0 \longrightarrow 1)$, i.e. two objects and one non-identity morphism. From the definition of geometric morphism it is then clear that $\mathbb{S}$-homotopies from $f$ to $g$ are in natural bijection with natural transformations from $f^{*}$ to $g^{*}$. Define a localic $\mathbb{S}$-homotopy from $f$ to $g$ to be a stably Frobenius adjunction $L \dashv R: \mathbf{L o c}_{\mathcal{F}} / \mathbb{S}_{\mathcal{F}} \rightleftarrows \mathbf{L o c}_{\mathcal{E}}$ such that $\left(0_{\mathbb{S}_{\mathcal{F}}}\right)^{*} R=f^{*}$ and $\left(1_{\mathbb{S}_{\mathcal{F}}}\right)^{*} R=g^{*}$. So,

Proposition 16.2. For any two toposes $\mathcal{F}$ and $\mathcal{E}$, the category of geometric morphisms from $\mathcal{F}$ to $\mathcal{E}$, i.e. $\mathfrak{T} \mathfrak{o p}(\mathcal{F}, \mathcal{E})$, is equivalent to the category of stably Frobenius adjunctions $L \dashv R: \mathbf{L o c}_{\mathcal{F}} \rightleftarrows \operatorname{Loc}_{\mathcal{E}}$ such that $R$ preserves the Sierpinski locale. 
The morphisms of $\mathfrak{T} \mathfrak{o p}(\mathcal{F}, \mathcal{E})$ are natural transformations (between the inverse images) and the morphisms of the category of stably Frobenius adjunctions are localic S-homotopies.

\section{BOUnDED GEOMETRIC MORPHISMS}

Consider a geometric morphism $f: \mathcal{F} \longrightarrow \mathcal{E}$. Section 4 of [T14] shows that condition (4') of Proposition 10.2 holds for the adjunction $\Sigma_{f} \dashv f^{*}$ if $f$ is bounded. On the other hand suppose that condition (4') of Proposition 10.2 holds. Then, as we have recalled that $\Sigma_{f} \dashv f^{*}$ is stably Frobenius, we know that $\mathbf{L o c}_{\mathcal{F}}$ is equivalent to $\left[\mathbb{G}, \mathbf{L o c}_{\mathcal{E}}\right.$ ] over $\mathbf{L o c}_{\mathcal{E}}$. By restricting to discrete objects we can then conclude that $f$ is equivalent to the canonical geometric morphism $\gamma: B_{\mathcal{E}} \mathbb{G} \longrightarrow \mathcal{E}$ which is well known to be bounded (e.g. B3.4.14(b) of J02 or see the definition of the relevant site in $\S 6$ of $[\mathrm{M} 88 \mathrm{a}]$ ) and so $f$ is bounded. In summary:

Lemma 17.1. A geometric morphism $f: \mathcal{F} \longrightarrow \mathcal{E}$ is bounded if and only if $\Sigma_{f} \dashv f^{*}: \mathbf{L o c}_{\mathcal{F}} \longleftrightarrow \operatorname{Loc}_{\mathcal{E}}$ satisfies one of the equivalent conditions of either Proposition 10.2 or Proposition 7.3.

This lemma captures the essence of the famous Joyal and Tierney result that any topos $\mathcal{F}$ bounded over $\mathcal{E}$ is equivalent to $B_{\mathcal{E}} \mathbb{G}$ for some open localic groupoid $\mathbb{G}$; see [T14] for more detail. We write $\mathfrak{B T} \mathfrak{o p}{ }^{i}$ for the 2-category whose objects are toposes, morphisms bounded geometric morphisms and whose 2-cells are natural isomorphisms.

Theorem 17.2. Let $\mathcal{S}$ be a topos with a natural numbers object. Sending bounded $p: \mathcal{E} \longrightarrow \mathcal{S}$ to $\Sigma_{p} \dashv p^{*}$ determines a pseudo-functor $\mathfrak{i}: \mathfrak{B T o p}^{i} / \mathcal{S} \longrightarrow T_{\text {Loc }_{\mathcal{S}}}$. This pseudo-functor preserves binary pseudo-products and is full and faithful, up to isomorphism.

The theorem can be adapted to show that all finite pseudo-limits are preserved; this is omitted in the interests of space.

Proof. The lemma shows how to construct $i$, though of course the construction of a localic groupoid for any bounded $p$ can be taken to be that defined via the well known Joyal-Tierney theorem [JT84. In particular we recall (C5.3 of [J02]) that given the bounded geometric morphism $\gamma: B_{\mathcal{E}} \mathbb{G} \longrightarrow \mathcal{E}$, the pseudo-functor returns $\left[\hat{\mathbb{G}}, \mathbf{L o c}_{\mathcal{S}}\right]$ where $\hat{\mathbb{G}}$ is the étale completion of $\mathbb{G}$. Therefore for any étale complete $\mathbb{G},\left[\mathbb{G}, \mathbf{L o c}_{\mathcal{S}}\right]$ is equivalent to $\mathfrak{i}(\gamma)$ for some bounded geometric morphism $\gamma$.

Since every bounded $p$ is equivalent to one of the form $\gamma: B_{\mathcal{E}} \mathbb{G} \longrightarrow \mathcal{E}$ to complete the proof of the assertion about binary pseudo-products, given Proposition 15.2, one just needs to check that $\mathbb{H} \times \mathbb{G}$ is étale complete if $\mathbb{G}$ and $\mathbb{H}$ are. But $\left[\mathbb{H} \times \mathbb{G}, \mathbf{L o c}_{\mathcal{S}}\right]$ is equivalent to $\left[\mathbb{H}^{*} \mathbb{G},\left[\mathbb{H}, \mathbf{L o c}_{\mathcal{S}}\right]\right]$ and Lemma 7.3 of M88a shows that $\mathbb{H}^{*} \mathbb{G}$ is étale complete if $\mathbb{G}$ is.

For the claim of 'full and faithful' use the the representation theorem of geometric morphisms as stably Frobenius adjunctions. Note that as $f^{*}$ preserves the Sierpiński locale for any geometric morphism $f, \mathbb{G}^{*} \mathbb{S}_{\mathcal{S}}$ is the Sierpiński locale of $\mathcal{E}$, where $\mathbb{G}$ is the localic groupoid associated with $\mathcal{E}$; similarly for $\mathcal{F}$. Therefore the right adjoint of $L \dashv R$ must preserve the Sierpinski locale for any adjunction $\left[\mathbb{H}, \mathbf{L o c}_{\mathcal{F}}\right] \rightleftarrows[\mathbb{G}, \mathbf{L o c} \mathcal{E}]$ provided it is over $\mathbf{L} \mathbf{o c} \mathcal{S}_{\mathcal{S}}$ 
Remark 17.3. One can expand this result to include natural transformations between geometric morphisms that are not necessarily isomorphisms; the morphisms of $T_{\mathbf{L o c}_{\mathcal{S}}}$ then need to be replaced with $\mathbb{S}$-homotopies. We note that if $\mathfrak{P}_{1}, \mathfrak{P}_{2}$ : $\mathbb{H} \longrightarrow \mathbb{G}$ are two Hilsum-Skandalis maps between stably Frobenius localic groupoids then a localic $\mathbb{S}$-homotopy is given by a Hilsum-Skandalis map $\mathfrak{P}: \mathbb{H} \longrightarrow \mathbb{G}$ with the additional data of an open a of $P$ (equivalently a locale map $a: P \longrightarrow \mathbb{S}$ ) such that $P_{1}$ is the open sublocale $a^{*} 1_{\mathbb{S}}$ of $P$ and $P_{2}$ is the closed sublocale $a^{*} 0_{\mathbb{S}}$ of $P$. This provides a localic characterisation of natural transformations between geometric mophisms (for bounded toposes at least).

Remark 17.4. It is unfortunate that this result uses Lemma 7.3 of [M88a as the proof of Lemma 7.3 requires discussion of sites and so we have not fully achieved a site-free description of the pullback of bounded geometric morphisms. However it is not unreasonable to expect that a site-free characterisation of étale completeness is possible and that this can be shown to be pullback stable without reference to sites. Indeed this has been done for localic groups, see Lemma 1.4 of [M88b].

\section{Further APPLications to GEOMETRIC MORPhisms}

In this section we prove a number of results about geometric morphisms using their representation as stably Frobenius adjunctions and applying our results about stably Frobenius adjunctions. The results are all essentially known; what is new is that they all follow from our earlier results about Frobenius adjunctions.

Proposition 18.1. Let $f: \mathcal{F} \longrightarrow \mathcal{E}$ and $g: \mathcal{G} \longrightarrow \mathcal{F}$ be two geometric morphisms. Then (i) $f g$ is localic(bounded) if $f$ and $g$ are, (ii) if $f g$ is localic then $g$ is localic; and, (iii) if $f g$ is bounded then $g$ is bounded.

Recall that a geometric morphism $f: \mathcal{F} \longrightarrow \mathcal{E}$ is localic if there is a locale $X$ in $\mathcal{E}$ and an equivalence $\mathcal{F} \stackrel{\simeq}{\longrightarrow} S h_{\mathcal{E}} X$ over $\mathcal{E}$. An equivalent condition on the pullback adjunction $\Sigma_{f} \dashv f^{*}$ is that there exists a locale $X$ in $\mathcal{E}$ and an equivalence

$\mathbf{L o c}_{\mathcal{F}} \stackrel{\simeq}{\longrightarrow} \operatorname{Loc}_{\mathcal{E}} / X$ over $\mathbf{L o c}_{\mathcal{E}}$; localic geometric morphisms are bounded (consider the trivial localic groupoid $\mathbb{X})$.

Proof. (i) Certainly if $f$ and $g$ are localic then so is $f g$ as a slice composed with a slice is again a sliced adjunction. Say $f$ and $g$ are bounded then Proposition 14.1 shows that the adjunction $\Sigma_{f g} \dashv(f g)^{*}$ satisfies the conditions of Proposition 7.3 and so $f g$ is bounded.

(ii) Say $f g$ is localic then (2.) of Lemma 4.2 shows that $\Sigma_{g} \dashv g^{*}$ is a slice and so $g$ is localic.

(iii) Proposition 14.3 .

Proposition 18.2. Any geometric morphism $f: \mathcal{F} \longrightarrow \mathcal{E}$ factors, uniquely up to isomorphsm, as a hyperconnected followed by a localic geometric morphism.

Recall that $f$ is hyperconnected if the canonical map $\Omega_{\mathcal{E}} \longrightarrow f_{*} \Omega_{\mathcal{F}}$ (i.e. the unique frame homomorphsm) is an isomorphism. An equivalent condition is that $\Sigma_{f}$ preserves 1. 
Proof. Repeating the remark just before Lemma 3.1 we know that there is a facotization:

$$
\operatorname{Loc}_{\mathcal{F}} \underset{\eta_{1}^{*}}{\stackrel{\Sigma_{\eta_{1}}}{\rightleftarrows}} \operatorname{Loc}_{\mathcal{F}} / f^{*} \Sigma_{f} 1 \underset{f_{\Sigma_{f}}^{*}}{\stackrel{\left(\Sigma_{f}\right)_{\Sigma_{f}}}{\rightleftarrows}} \operatorname{Loc}_{\mathcal{E}} / \Sigma_{f} 1 \underset{\left(\Sigma_{f} 1\right)^{*}}{\stackrel{\Sigma_{\Sigma_{f} 1}}{\rightleftarrows}} \operatorname{Loc}_{\mathcal{E}}
$$

Because $\Sigma_{f} \dashv f^{*}$ is stably Frobenius and pullback adjunctions are stably Frobenius the composite of the first two adjunctions, $\left(\Sigma_{f}\right)_{\Sigma_{f} 1} \Sigma_{\eta_{1}} \dashv \eta_{1}^{*} f_{\Sigma_{f}}^{*}$, is stably Frobenius. But the right adjoint of this composite adjunction preserves the Sierpiński locale and so it must be the pullback adjunction of a geometric morphism, $\bar{f}$; and as $\left(\Sigma_{f}\right)_{\Sigma_{f} 1} \Sigma_{\eta_{1}} 1=1, \bar{f}$ is hyperconnected and this completes the proof of the existence of the factorization because pullback adjunctions correspond to the pullback adjunctions of localic geometric morphisms.

Let us say we have another factorization of $f$ as $\mathcal{F} \stackrel{p}{\longrightarrow} S h_{\mathcal{E}} X \longrightarrow \mathcal{E}$ with $p$ hyperconnected. Because $\Sigma_{p} 1 \cong 1$ it follows that $X \cong \Sigma_{f} 1$ (use $\Sigma_{X} 1 \cong X$ ) and so there is an equivalence $\psi: \operatorname{Loc}_{\mathcal{E}} / X \stackrel{\simeq}{\longrightarrow} \operatorname{Loc}_{\mathcal{E}} / \Sigma_{f} 1$ over $\mathbf{L o c}_{\mathcal{E}}$. To complete it just needs to be checked that $\psi p$ is isomorphic to $\bar{f}$. But any object $Y_{g}$ of $\operatorname{Loc}_{\mathcal{E}} / X$ can be written as an equalizer

$$
Y_{g} \longrightarrow X^{*} Y \underset{\Delta_{X} !}{\stackrel{X^{*} f}{\rightleftarrows}} X^{*} X
$$

and so $p^{*} Y_{g}$ is determined by the image of this equalizer. Note that $1 \stackrel{p^{*} \Delta_{X}}{\longrightarrow} p^{*}\left(X^{*} X\right)$ is the double adjoint transpose (via $\Sigma_{p} \dashv p^{*}$ and $\Sigma_{X} \dashv X^{*}$ ) of the identity $\Sigma_{X} \Sigma_{p} 1 \stackrel{I d_{X}}{\longrightarrow} \Sigma_{X} \Sigma_{p} 1$; but this identity is isomorphic to the identity $\Sigma_{\Sigma_{f} 1} \Sigma_{\bar{f}} \stackrel{I d_{\Sigma_{f}} 1}{\longrightarrow} \Sigma_{\Sigma_{f} 1} \Sigma_{\bar{f}} 1$ and so has isomorphic double adjoint transpose (now via $\Sigma_{\bar{f}} \dashv \bar{f}^{*}$ and $\Sigma_{\Sigma_{f} 1} \dashv \Sigma_{f} 1^{*}$ ). It follows that $p^{*} \Delta_{X}$ is isomorphic to $\bar{f}^{*} \Delta_{\Sigma_{f} 1}$.

Proposition 18.3. The hyperconnected-localic factorization of a bounded geometric morphism along a bounded geometric morphism is pullback stable.

Proof. If $X$ is an object of a category $\mathcal{C}$ and $\mathbb{G}$ an internal groupoid, then there is an isomorphism between $[\mathbb{X} \times \mathbb{G}, \mathcal{C}]$ and $[\mathbb{G}, \mathcal{C}] / \mathbb{G}^{*} X$. So that localic geometric morphisms are pullback stable follows from the description of product given in Proposition 15.2. For the pullback stability of hyperconnected geometric morphisms, this follows as the diagram $\left(^{*}\right)$ in the proof of Proposition 15.2 satisfies Beck-Chevalley (both ways round). That the diagram satisfies Beck-Chevalley is clear from the explicit description of $\Sigma_{\pi_{2}}$ given in (i) of Proposition 10.3

Proposition 18.4. Open and proper bounded geometric morphisms are pullback stable (along bounded geometric morphisms).

Proof. A geometric morphism is open(proper) if and only if the localic part of its hyperconnected-localic factorization is open(proper); see C3.1.9 (preamble to $\mathrm{C} 3.2 .12$ ) of [J02]. The result then follows from the previous proposition as it is well known that $f^{*}: \mathbf{L o c}_{\mathcal{E}} \longrightarrow \mathbf{L o c}_{\mathcal{F}}$ preserves both open and proper locales for any geometric morphism $f: \mathcal{F} \longrightarrow \mathcal{E}$.

Proposition 18.5. For any two toposes $\mathcal{F}$ and $\mathcal{E}$, bounded over some base topos $\mathcal{S}$, the category of geometric morphisms from $\mathcal{F}$ to $\mathcal{E}$ over $\mathcal{S}$ (with natural isomorphisms as morphisms) is equivalent to the category of Hilsum-Skandalis maps from $\mathbb{H}$ to $\mathbb{G}$ where $\mathbb{H}(\mathbb{G})$ is the localic groupoid associated with $\mathcal{F}(\mathcal{E})$. 
Proof. Immediate from our main result (Theorem[11.3) and our earlier result, contained in Theorem 17.2, that $\mathfrak{i}$ is full and faithful up to isomorphism.

Proposition 18.6. For any bounded $p: \mathcal{E} \longrightarrow \mathcal{S}$ there is a localic groupoid $\mathbb{G}=\left(G_{1} \longrightarrow G_{0}, \ldots\right)$ such that for every point $x: 1 \longrightarrow G_{0}$ there is a geometric morphism $p_{x}: \mathcal{S} \longrightarrow \mathcal{E}$ over $\mathcal{S}$ (i.e. a point of $\mathcal{E}$ ). The full subgroupoid of $\mathfrak{B T o p}^{i}(\mathcal{S}, \mathcal{E})$ consisting of all $p_{x} s$ is isomorphic to the groupoid (in $\mathcal{E}$ ) of points of $\mathbb{G}$.

Proof. Proposition 11.4.

\section{CONCluding REMARKS}

Whilst writing this paper the idea of changing the title to 'Everything you always wanted to know about a cartesian category (but were afraid to ask)' occurred to me. At the outset I did not think that generalities on cartesian categories were going to produce any work of substance. The results would either be well known or just not true at that level of generality. The core result on the representation of HilsumSkandalis maps was clearly going to require some work, but I was surprised by the depth of the more basic lemmas, notably our earlier ones on slicing adjunctions (e.g. Lemma 3.1) and, as should be apparent from the exposition, the Proposition characterising connected component adjunctions. So, at the very least, this paper should be of use to those who want to see basic results on sliced adjunctions, stably Frobenius adjunctions and groupoids expressed at the greatest possible level of generality ('greatest' because the cartesian structure is needed to express the stably Frobenius condition and in the definition of internal groupoids).

Open surjections in the category of locales are effective descent morphisms and are stable under all the relevant constructions (basically, pullback). So by replacing 'effective descent morphism' with 'open surjection' one can return to a topological 'terra firma' should that be required. But our essential point is that the relationships persist at a greater level of generality (cartesian categories) and so should be of general interest. The additional generality is not vacuous because proper surjections in the category of locales provide a non-trivial example, different to open surjections.

The essence of the main result on Hilsum-Skandalis maps is already in [T15, from where the ideas of this paper stem. That localic Hilsum-Skandalis maps represent geometric morphisms is implicit in the literature as they are known to correspond to the morphisms of the category of fractions of open groupoids, and geometric morphisms are known to be represented by the morphsms of this category of fractions; see Section 7 of M88a. What is new for this paper is that we have provided a new explicit charcterisation of the morphisms of the relevant category of fractions: they are the stably Frobenius adjunctions.

It has not been possible here to provide a localic characterisation of étale completeness of localic groupoids. Therefore some extra work remains to support any assertion that we have 'done topos theory without sites'. But I think it is fair to say that most of the heavy lifting has been done above, notably with Proposition 15.2. The idea of reasoning about categories of locales to recover known aspects of topos theory is well motivated, but needs to be finished! 
However, what I believe to be of greater interest is working out what happens when we forget about étale completeness. The paper [T16] shows that $[\mathbb{G}$, Loc] behaves like a perfectly good category of locales even if $\mathbb{G}$ is not étale complete and so $[\mathbb{G}, \mathbf{L o c}]$ is not actually a category of locales over some topos. What we have shown here is that the theory of 'geometric morphisms'(=stably Frobenius adjunctions; let's call them Frobenius morphisms) works at a greater level of generality. There is an account of hyperconnected-localic factorization, boundedness, pullbacks, open and proper maps and, e.g. [T12, aspects of internal lattice theory, all with 'categories of locales' taking on the role of toposes and Frobenius morphisms taking on the role of geometric morphisms. And the key point of this paper is that Frobenius mophisms can be represented as Hilsum-Skandalis maps (i.e. generalised principal bundles). There is further work required to see how useful this new context is. As a final idea consider the question 'What should the natural numbers object look like in this context?' We propose the following axiom of infinity: there exists an internal groupoid $\mathbb{G}$ and a natural categorical equivalence $\operatorname{Dis}_{\mathcal{D}} \simeq T_{\mathcal{C}}(\mathcal{D},[\mathbb{G}, \mathcal{C}])$ for each $\mathcal{D}$ over $\mathcal{C}$. In other words, $\mathbb{G}$ classifies discrete objects. Restricting to toposes we get a characterisation of the natural numbers (B4.2.11 of [J02]). What happens if we don't make that restriction?

\section{ACKNowledgement}

This paper is dedicated to my son, Nathan.

\section{REFERENCES}

[J02] Johnstone, P.T. Sketches of an elephant: A topos theory compendium. Vols 1, 2, Oxford Logic Guides 43, 44, Oxford Science Publications, 2002.

[JT84] Joyal, A. and Tierney, M. An Extension of the Galois Theory of Grothendieck, Memoirs of the American Mathematical Society 309, 1984.

[M88a] Moerdijk, I. The classifying topos of a continuous groupoid. I, Transactions of the American Mathematical Society, Volume 310, Number 2, (1988) 629-668.

[M88b] Moerdijk, I. Morita equivalence for continuous groups, Math. Proc. Camb. Phil. Soc. 103 (1988) 97-115

[Mr96] Mrcun, J. Stability and invariants of Hilsum-Skandalis maps, Ph.D. thesis, Utrecht University, (1996).

[T16] Townsend, C.F. Stability of Properties of Locales Under Groups. Appl Categor Struct (2016). doi:10.1007/s10485-016-9430-6

[T15] Townsend, C.F. Principal bundles as Frobenius adjunctions with application to geomtric morphisms. Math. Proc. Camb. Phi. 159 (2015), 433-444.

[T14] Townsend, C.F. A short proof of the localic groupoid representation of Grothendieck toposes. Proceedings of the American Mathematical Society 142.3 (2014): 859-866.

[T12] Townsend, C.F. Aspects of slice stability in Locale Theory Georgian Mathematical Journal. Vol. 19, Issue 2, (2012) 317374.

[T10] Townsend, C.F. A representation theorem for geometric morphisms. Applied Categorical Structures. 18 (2010) 573-583. 